\title{
Animate Experience \\ The Architectural Potential of Digital Media in Duration,
}

Mark T. Rosen, B.A.S.

A Thesis Submitted to the Department of Graduate Studies

In Partial Completion of the Requirements for the Degree of

\author{
Master of Architecture
}

Carleton University

Ottawa, Ontario

29.09 .05

(C) Copyright 2005, Mark T. Rosen 


\section{Abstract}

Our changing experiential condition is a result of the synthesis of our perceptions and our memories. The addition to memory of each passing moment conditions perception in the present, thereby effecting a transformation of our present experience. This transformation of experience over time is characteristic of Bergsonian duration. The invention of animated representation aligns the passage of time in the image and the viewer's own sense of duration. Thus, animated representation expresses virtual experience.

This thesis investigates the digital medium, specifically through the invention of animated experience. The primary interest is the application of this concept to architectural design, with emphasis placed on the incorporation of the digital tool at three stages in the development of the project: the investigation of site, an experiential intervention in the site, and finally, the translation of an architectural proposal for the site. 


\section{Acknowledgements}

To my parents, for their constant support and inspiration. 


\section{Table of Contents}

List of Figures vi vi

Introduction $\quad 1$

Technological Conditioning of Perception $\quad 4$

The effects of invention on perception, memory, duration, experience.

Recreating/Representing the Real

Mobility and the real, intuition, and animation.

Real: Possible: Virtual

Actualization of virtuality, realization of possibility.

Animate Experience

Synthesis of duration, animation. The enhanced digital tool.

Translation

Experience to construction.

The Project

Site

Experience 
Conclusion

Bibliography 


\section{List of Figures}

Fig. 1 House Prototype in Long Island. From: Greg Lynn, Animate Form (New York: Princeton Architectural Press, 1999) 153.

Fig. 2 House Prototype in Long Island. From: Lynn, Animate Form, 160-161.

Fig. 3 House Prototype for Long Island. From: Greg Lynn, Animate Form (New York: Princeton Architectural Press, 1999) 160.

Fig. 4 Stereolithography model of House Prototype in Long Island. From: Lynn, Animate Form, 161.

Fig. 5 Eisenman's Virtual House. From: Luca Galofaro, Digital Eisenman: An Office of the Electronic Era, trans. Lucinda Byatt (Boston: Birkhäuser, 1999) 65.

Fig. 6 Genesis of the Virtual House. From: Galofaro, Digital Eisenman: An Office of the Electronic Era, 61.

Fig. 7 Sections of Eiseman's Virtual House. From: Galofaro, Digital Eisenman: An Office of the Electronic Era, 68-69.

Fig. 8 Plans of Eisenman's Virtual House. From: Galofaro, Digital Eisenman: An Office of the Electronic Era, 68-69.

Fig. 9 Digital model of Hadid's Rosenthal Center for Contemporary Art. From: "Building Motion: The Architecture of Zaha Hadid", Place Tower Arts Center - Exhibitions (Sept. 10, 2005) < http://www.pricetower.org/art/Archive/zaha.html>.

Fig. 10 Digital Model of Hadid's Rosenthal Center for Contemporary Arts. From: "The Lois \& Richard Rosenthal Center for Contemporary Art, Cincinnati”, DesignBoom 
(Sept. 10, 2005) < http://www.designboom.com/portrait/zaha_c.html>.

Fig. 11 Digital Model of Hadid's Rosenthal Center for Contemporary Arts. From: "The Lois \& Richard Rosenthal Center for Contemporary Art, Cincinnati”, DesignBoom (Sept. 10, 2005) < http://www.designboom.com/portrait/zaha_c.html>.

Fig. 12 Digital Model of interior of Hadid's Rosenthal Center for Contemporary Arts.

From: "The Lois \& Richard Rosenthal Center for Contemporary Art, Cincinnati", DesignBoom (Sept. 10, 2005) < http://www.designboom.com/portrait/zaha_ c.html>.

Fig. 13 Interior of Hadid's Rosenthal Center for Contemprary Arts. From: ArcoWeb (Sept. $10,2005)<$ http://www.arcoweb.com.br/arquitetura/arquitetura466.asp>.

Fig. 14 Hadid's Rosenthal Center for Contemporary Arts, Cincinnati. From: ArcoWeb (Sept. $10,2005)$ < http://www.arcoweb.com.br/arquitetura/arquitetura466.asp>

Fig. 15125 Hickory Street, Ottawa, Ontario, Canada. From: Google Earth, Sept 10, 2005.

Fig. 16 View of Dow's Lake from near the site.

Fig. 17 Park at the corner of Champagne avenue and Beech street.

Fig. 18 Digital site model.

Fig. 19 Digital site model.

Fig. 20 Digital site model.

Fig. 21 Approaching Carling avenue. Digital site model.

Fig. 22 Carling avenue. Digital site model.

Fig. 23 View towards Dow's Lake. Digital site model.

Fig. 24 Looking towards Carling avenue. Digital site model.

Fig. 25 The park at Champagne avenue and Beech street. Digital site model.

Fig. 26 Pathway on the east side of the O-Train track. Digital site model.

Fig. 27 Plan view of site. Digital site model.

Fig. 28 View towards the city to the northeast. Digital site model.

Fig. 29 Digital experiential model.

Fig. 30 Digital experiential model. 
Fig. 31 Digital experiential model.

Fig. 32 Digital experiential model.

Fig. 33 Digital experiential model.

Fig. 34 Digital experiential model.

Fig. 35 Digital experiential model.

Fig. 36 Digital experiential model.

Fig. 37 Digital experiential model.

Fig. 38 Digital experiential model.

Fig. 39 Digital experiential model.

Fig. 40 Digital experiential model.

Fig. 41 Digital experiential model.

Fig. 42 Digital architectural model.

Fig. 43 Digital architectural model.

Fig. 44 Digital architectural model.

Fig. 45 Digital architectural model.

Fig. 46 Digital architectural model.

Fig. 47 Digital architectural model.

Fig. 48 East elevation. Digital architectural model.

Fig. 49 South elevation. Digital architectural model.

Fig. 50 West elevation. Digital architectural model.

Fig. 51 North elevation. Digital architectural model.

Fig. 52 Gallery interior. Digital architectural model.

Fig. 53 Terrace level gallery entrance. Digital architectural model.

Fig. 54 Rooftop atrium. Digital architectural model. 
Fig. 55 Stairwell. Digital architectural model.

Fig. 56 Stair to terrace. Digital architectural model.

Fig. 57 Building at dusk. Digital architectural model. 


\section{Introduction}

I was soon struck by what seemed at the time the peculiar disadvantage under which architects labored, never working directly with the object of their thought, always working at it through some intervening medium...

- Robin Evans

The tool of the architect is representation; as Evans observes, representation is the mode through which architectural knowledge is communicated. As a result, the limit of architectural expression is necessarily the limit of architectural representation, and architectural exploration is necessarily concerned with the invention of new modes of representation. It is through this that the scope of possible action in architecture is widened.

In recent years, the obsolescence of traditional modes of representation by computer

\footnotetext{
Evans, "Translations from Drawing to Building" Translations from Drawing to Building and Other Essays (Cambridge, Massachusetts: The MIT Press, 1997) 156.
} 
modeling technology has been accompanied by a shift in our perception of architectural possibility. Architects such as Peter Eisenman and Greg Lynn have experimented with digital technology as a generator of form, allowing for the expression and resolution of complex geometries. Each uses animation as a medium for the expression of transformation, delineating the progression of the final form from its original state. Through some of their theoretical projects, this fascination with form has resulted in architectural projects that exclude the viewer in favor of an objectified, exterior vantage point. In an effort to reintegrate the viewer in the digital design process, a different emphasis must be sought.

The viewer's relationship to architectural representation involves an act of projection. This projection requires the viewer to imagine the eventual reality of spatial representation, and attempt to construct in one's mind some concept of the experience of inhabitation. Because experience is synthesized in the present, in every passing moment, it is continuously in a state of transformation, a condition that immobile spatial representation is unable to emulate. Conversely, the capacity for animation in digital representation enables the creation of a mobile viewpoint. This representation of movement in time creates what Gilles Deleuze refers to in cinema as a "mobile section" 2 that aligns itself with the viewer's own sense of time, thereby facilitating a synthesis of experience. Since experience is evoked by the physical presence of architecture, an investigation of the potential for representing experience reveals a new design process, integrating the viewer with the digital medium. The animated digital medium embodies a virtual experience of space that the viewer accesses in the present

2 Deleuze, CINEMA 1, The Movement-Image, trans. H. Tomlinson and Barbara Habberjam (Minneapolis: University of Minnesota Press, 1986) 11. 
moment; this ability to represent animated experience permits the invention of architecture through experience. 


\section{Technological Conditioning of Perception}

But, then, I cannot escape the objection that there is no state of mind, however simple, which does not change every moment, since there is no consciousness without memory, and no continuation of a state without the addition, to the present feeling, of the memory of past moments. It is this which constitutes duration. ${ }^{3}$

- Henri Bergson

Bergson's concept of duration lays a foundation on which we are able to build an understanding of our own changing experiential condition. This condition is our relationship to our surroundings: our senses and memories combine to define our knowledge through experience. Experience is a state of conscious awareness, and is not static, but mobile and momentary. It transforms as each moment slips into memory, subsequently and immediately contributing to our awareness of the present. In the continuous present moment, it is the addition of our physical sensory inputs to our evolving memories that defines our

3 Henri Bergson, An Introduction to Metaphysics, (United States: The Liberal Arts Press Inc., 1955), 40. 
experience. Because of this, experience is not a measurable quantity. Rather, it is ephemeral and subjective, and unique for each individual. The intangible nature of experience is due to two things: first, to the complexity/multiplicity of both our senses and our memories, and second, to its relationship to duration and Bergson's concept of a singular moment of the present. Sensorial multiplicity is plain to understand: five physical senses contribute in varying amounts to our mind's recognition of our environment. In memory, complexity and multiplicity describe the ongoing transformation of past moments in the mind. Because of our inability to constantly absorb in full the details of our surroundings, each memory is subject to a filtering process through which unessential details are removed and forgotten. In essence, the memories that are formed are incomplete images. The recollection of past events relies on the recombination of these fragments, a process which is influenced by present circumstances. Reciprocally, the influence of these reassembled memories on our awareness of the present moment conditions our immediate experience. These seemingly oppositional processes occur simultaneously rather than successively. Bergson observes this in duration:

The cerebral mechanism is arranged just so as to drive back into the unconscious almost the whole of this past, and to admit beyond the threshold only that which can cast light on the present situation or further the action now being prepared... ${ }^{4}$

Further to this, Bergson declares that no two successive moments are ever identical, since with each moment "memory [of previous moments] is there, which conveys something

4 Bergson, Creative Evolution, trans. Arthur Mitchell (London: MacMillan and Co. Limited, 1913) 5. 
of the past into the present." Since no two moments in time are identical, and we retain an awareness of past moments even as we continue forward in time, our experience is in constant flux.

Internal (mental) and external (physical) perception are the mediators of our experience: the transformation of experience relies continuously on the changing states of each. This derivation of experience from perception is observed by Immanuel Kant, who states:

"Experience thus is a synthesis of perceptions which itself is not contained in perception but contains the synthetic unity of the manifold of perceptions in one consciousness." ${ }^{6}$ Therefore, experience is not defined exclusively by either external or internal perception, but rather by the combination of both in one mind. Internal perception is tied to memory, which is "continually swelling with the duration which it accumulates" While this accumulation of successive moments is ongoing, the parallel function of memory is to recall past experiences that inform our interpretation of the present situation. This second function is knowledge; it is the accumulation of perceptions in memory, in other words, the retention in memory of accumulated duration. From Bergson:

We note that [memory's] primary function is to evoke all those past perceptions which are analogous to the present perception, to recall to us that decision which is the most useful. ${ }^{8}$

\footnotetext{
5 Bergson, Creative Evolution, 2.

6 Immanuel Kant, Critique of Pure Reason, trans. Wolfgang Schwarz (Germany: Scientin Verlag Aalen, 1982) 73.

Bergson, Creative Evolution, 2.

8 Bergson, Matter and Memory, trans. N. M. Paul and W. S. Palmer (London: George Allen and Unwin Ltd.) 228.
} 
This internal perception, as it contributes to the ongoing synthesis of experience, is the application of knowledge to external perception. By filtering through past actions and situations, memory brings to the fore those moments which best inform our present state. As memory accumulates through duration, it contributes to knowledge in two methods: first, as habit, and second, as true memory. While habit conditions a primarily instinctual response to a situation, true memory allows us to deliberately recall specific past occurrences ${ }^{9}$. In essence, knowledge is the combination of our conscious and unconscious awareness of the world. This awareness, which is conditioned by each new experience, is ever-changing; our knowledge is ever-expanding. As it expands, it in turn contributes in new ways to our internal perception in the present, thereby affecting our experience of the same.

External perception is grounded in the senses; the tactile reality of our environment is accessed when it is touched or heard or seen. The extension of these faculties has long been one of our primary preoccupations as human beings, and has inspired the invention of technologies that involve themselves in the relationship of the body to the environment. Eyeglasses, for example, extend our visual capacity: they are a prosthetic extension of the eye. The invention of technology (and indeed architecture) is concerned with this prosthetic mediation of our relationship to the natural world. As Elizabeth Grosz suggests, technology is "the inevitable result of the encounter between life and matter...the consequence of the

\footnotetext{
9 For a more in depth examination of the distinctions between these types, read Bergson, Matter and Memory, 150-151.
} 
living's capacity to utilize the nonliving (and the living) prosthetically." ${ }^{10}$ Because it affects our external perception, prosthetic technology conditions experience.

Since its inception, technology's primary function has been prosthetic, aiming to alter the relationship of the human body to its surrounding environment. This intervention is more apparent in some forms of technology than in others; a car, for example, dramatically increases one's speed of travel across land. It does, however, exist in all technologies. The technology of medicine, for example, may alter the body at a cellular level, increasing resistance to harmful environmental conditions. This, then, is perhaps the most fundamental definition of technology: a physical innovation that serves to alter the body/environment relationship.

When the effects of new technologies are observed, a mental adjustment occurs; it is the psychological equivalent of a prosthetically enhanced condition. Petran Kockelkoren describes this psychological conditioning in terms of "decentering" and "recentering", where the former refers to the disorientation of the mind in light of a new technology, and the latter refers to the mental realignment that necessarily occurs in order to coexist with the same ${ }^{11}$. He defines this conditioning by the term "technoesis", which denotes "an act of cognition via technology or the technologically mediated formation of images and conferral of meaning"12. "Technoesis" describes our present relationship to the world through technology. I say

\footnotetext{
${ }_{10}$ Elizabeth Grosz, Architecture from the Outside (Cambridge: The MIT Press, 2001) 176.

11 Petran Kockelkoren, Technology: art, fairground and theater (Rotterdam: NAi Publishers, 2003) 9-19.

12 Kockelkoren, Technology: art, fairground and theater, 34.
} 
"through technology" because the awareness of new technology expands our knowledge by extending our perception of the world prosthetically. Because this prosthetic intervention affects us on a psychological level as well as on a physical level, technology is in fact an extension of one's self rather than an external apparatus or tool. Marshall McLuhan, in his work entitled Understanding Media: The Extensions of Man, writes that "the personal and social consequences of any medium - that is, of any extension of ourselves - result from the new scale that is introduced into our affairs by each extension of ourselves, or by any new technology." 13 This progression of the human psyche is demonstrated by Kockelkoren, who discusses public reaction to the advent of rail travel:

...the hikers see what they smell and what they hear in a harmony of the senses known as synaesthesia. However, as soon as the step into a carriage and the locomotive starts up, that synaesthesia disintegrates. What they smell is not what they see, they hear the rattle of the wheels on the rails, the horizon slips smoothly by, devoid of any relation to their own body ${ }^{14}$

Confronted by this unfamiliar technology, the passenger of the train undergoes a decentering of their knowledge of the world, as their relationship to their surroundings is transformed by the unfamiliar speed of the train. The dissolution of an understood synaesthetic condition (the hiker) reveals the necessity for a new experiential awareness in the passenger. As Kockelkoren explains, our minds are at first sent reeling in light of such new technology, but

\footnotetext{
${ }_{13}$ Marshall McLuhan, Understanding Media: The Extensions of Man (New York: McGraw-Hill Book Company, 1964) 7. McLuhan's inclusion of technology in his discussion of media underlines their basic relationship: new media is the direct result of new technology, where photography requires the camera, painting the brush, cinema the projector. A discussion of technology necessarily includes consideration of its associated media, since both contribute to the decentering and recentering of our knowledge.

${ }^{14}$ Kockelkoren, Technology: art, fairground and theater, 16.
} 
as it becomes integrated into culture, the mind is able to realign itself with its new perception of the world. Rail travel no longer disorients the passenger who has an awareness of the physical relation of the train to the ground, and of themself to the train. Thus we see that the physical influence of technology extends to the mind, altering our mental perception as well as our physical condition. Grosz elaborates:

It is not that technologies mediate between the human and the natural - for that is to construe technology as somehow outside either the natural or the human (which today is precisely its misrepresented place) instead of seeing it as the indefinite extension of both the human and the natural and as their point of overlap... ${ }^{15}$

Because this prosthetic engagement with technology reveals the world to us in new ways, it expands our awareness through decentering and recentering; thus, the effects of new technology expand the limits of knowledge.

This expansion of knowledge through technology, when coupled with Bergson's theory of duration, demonstrates the irreversible nature of our changing experiential condition. Once exposed to a new technology, an awareness of its effects is imprinted on our understanding of the world. Consciously or unconsciously, this awareness is brought to bear on the present moment; this is the function of memory. Because of the accumulative nature of memory, we cannot revert back to a former state of unawareness, short of physically altering our state of the mind through sickness or injury. Once contained in memory, the knowledge

15 Grosz, Architecture from the Outside, 177. 
of new technologies is applied through our internal perception to our present experience.

Our changing experiential condition, then, is directly influenced by perception, which is transformed through technology. 


\section{Re-creating/Representing the Real}

The material world determines the immaterial world... ${ }^{16}$

- Jacques Herzog

The real which surrounds us is complex: Bergson states that "fixed concepts may be extracted by our thought from mobile reality; but there are no means of reconstructing the mobility of the real with fixed concepts. ${ }^{17}$ The intellectual act of understanding the real is rooted in the act of analysis, which distills complex mobility into static immobility. Bergson discusses this tendency with regards to motion in space:

Along the whole of this movement we can imagine possible stoppages; these are what we call the positions of the moving body, or the points by which it passes. But with these positions, even with an infinite number of them, we shall never make movement. ${ }^{18}$

$\overline{16}$ Jacques Herzog, "http://virtualhouse.ch", ANY (no. 19-20, 1997) 20.25.

17 Bergson, An Introduction to Metaphysics, 51.

18 Bergson, An Introduction to Metaphysics, 42. 
Thus we realize that our understanding of the world is based on the analysis of fixed immobilities, a process facilitated by technology, while the world is in a constant state of mobility. Grosz discusses digital technology in this role: "contemporary binarization and digitalization are simply the current versions of this tendency to the clear-cut, the unambiguous, the oppositional or binary impulses of the intellect"19. Intellectual analysis operates on these packages of static information. Bergson's example of a moving body demonstrates that analysis necessarily reduces motion to a finite number of fixed moments which capture the body in various successive points in time and space. Each of these points refers to a single position of the moving body, a position that is moved through rather than occupied. ${ }^{20}$ The reduction of motion into static frames allows the intellect to analyze its nuances and influences. Because of this, analysis necessarily operates in the past, in memory, where these static frames remain as images of previous moments.

Intuition, on the other hand, is a function of the present. Bergson uses this term to explain the instantaneous relationship of our consciousness to the present moment: "analysis operates always on the immobile, whilst intuition places itself in mobility, or, what comes to the same thing, in duration." ${ }^{21}$ Intuition, therefore, immediately grasps movement in its complex, mobile state. Since our present experience is synthesized in duration, it follows that intuition conditions experience as a part of our perception. Thus our relationship to the real is twofold:

\footnotetext{
19 Grosz, Architecture from the Outside, 180.

20 Bergson, An Introduction to Metaphysics, 42.

21 Bergson, An Introduction to Metaphysics, 41.
} 
intuitively, through duration, and intellectually, through analysis.

The analytic reduction of motion reveals its complexity: it isn't until we try to reconstruct movement out of a finite number of fixed points that we realize the futility of the task. It is also at this juncture that we gain an appreciation for the purity of the actual motion that is intuitively grasped in an instant by the mind. This in turn leads Bergson to suggest that motion is in fact the purest simplicity, understood instantaneously through intuition, and that the complexity of motion only becomes apparent when one attempts to reconstruct it from immobilities. This realization extends beyond motion to encompass all of the mobile real. Our dissection of the real, with the aid of technological innovation, clarifies the phenomena that we observe by repackaging them into immobile units: the reflections of light off of rippling water, for example, can be traced back to their source and understood through calculations taken at a fixed moment in time. However, the re-creation through calculation of these reflections, complete with their transformation from moment to moment, the influence of air currents on the water surface, and any other number of unknowable contributing factors, cannot capture the exact condition of the real that it mimics. Thus we become aware, through this attempt, of the irreducible complexity of the real.

Digital technology, specifically software designed for computer modeling, is applied by many users in an attempt to reduce the gap between the mobile real and the represented real in cyberspace. Increasingly powerful hardware and software combinations, capable of solving 
increasingly complex calculations, take their cues from the real that they seek to emulate. The effects of caustic reflections, for example, are observed in the physical world, and serve as the basis for the caustic calculations that the software performs on the objects and light sources in the digital world. This reveals the analytic nature of cyberspace. As Grosz writes, digitization apprehends the real "by decomposing the analog or continuous-currents into elements, packages, or units, represented by the binary code, and then recomposing them through addition: analysis then synthesis." 22 But this synthesis of dissected packages can never aspire to the unity of the real. What, then, is to be gained from the common practice of digitally modeling the space of reality; merely an understanding of its complexity? The futility of an attempt to recreate the mobility of the real suggests to some that digital technology is merely an escape from reality, one that frees us from the constraints of physicality and provides an arena for play. Paul Virilio perceives it thus:

...what the networks offer today to the new individualists is less communication than a kind of "emergency exit" that allows them to free themselves from their real dimensions. This transgression of physical laws is a delirium, known in psychiatry as 'total performance syndrome', in other words, delusions of grandeur. ${ }^{23}$

When used as a tool for the attempted re-creation of the real, the application of this digital technology is restricted by our existing knowledge; that is to say, we are able to apply the tool only in an attempt to capture that which we already understand. Because of this, such application is well suited to the presentation and final visualization of completed

\footnotetext{
22 Grosz, Architecture from the Outside, 181.

${ }^{23}$ Paul Virilio, "Cybernetics \& Society", $\underline{\text { ANY }}$ (no. 19-20, 1997) 19.19.
} 
architectural designs, allowing the architect to convey to the viewer in great detail images of space, materiality, and light. And yet, relegated to the role of producing the after-image, the digital tool in this capacity contributes little to the design process itself. Is there more to be gained from this medium if an alternative approach is considered?

In architectural design, traditional methods of drawing and physical modeling are employed not as tools to re-create, but to represent. Similarly, rather than trying to recreate a perceived reality, the digital model should be used to investigate and represent architectural concepts from within the design process. This integration has been adopted by some already. Lynn, in his book Animate Form, discusses the application of computer technology to architecture as a way to re-conceive the space of design:

Rather than as a frame through which time and space pass, architecture can be modeled as a participant immersed within dynamical flows. In addition to the special-effects and animation industries, many other disciplines such as aeronautical design, naval design, and automobile design employ this animate approach to modeling form in a space that is a medium of movement and force. $^{24}$

Lynn demonstrates this approach in his theoretical project titled "House Prototype in Long Island". The translation of the physical site into a series of forces in the digital model introduces the computer into the design at the outset of the process. He explains that "[the] site is mapped based on visual obstacles and visual attractors using forces of various

${ }_{24}$ Greg Lynn, Animate Form (New York: Princeton Architectural Press, 1999) 11. 


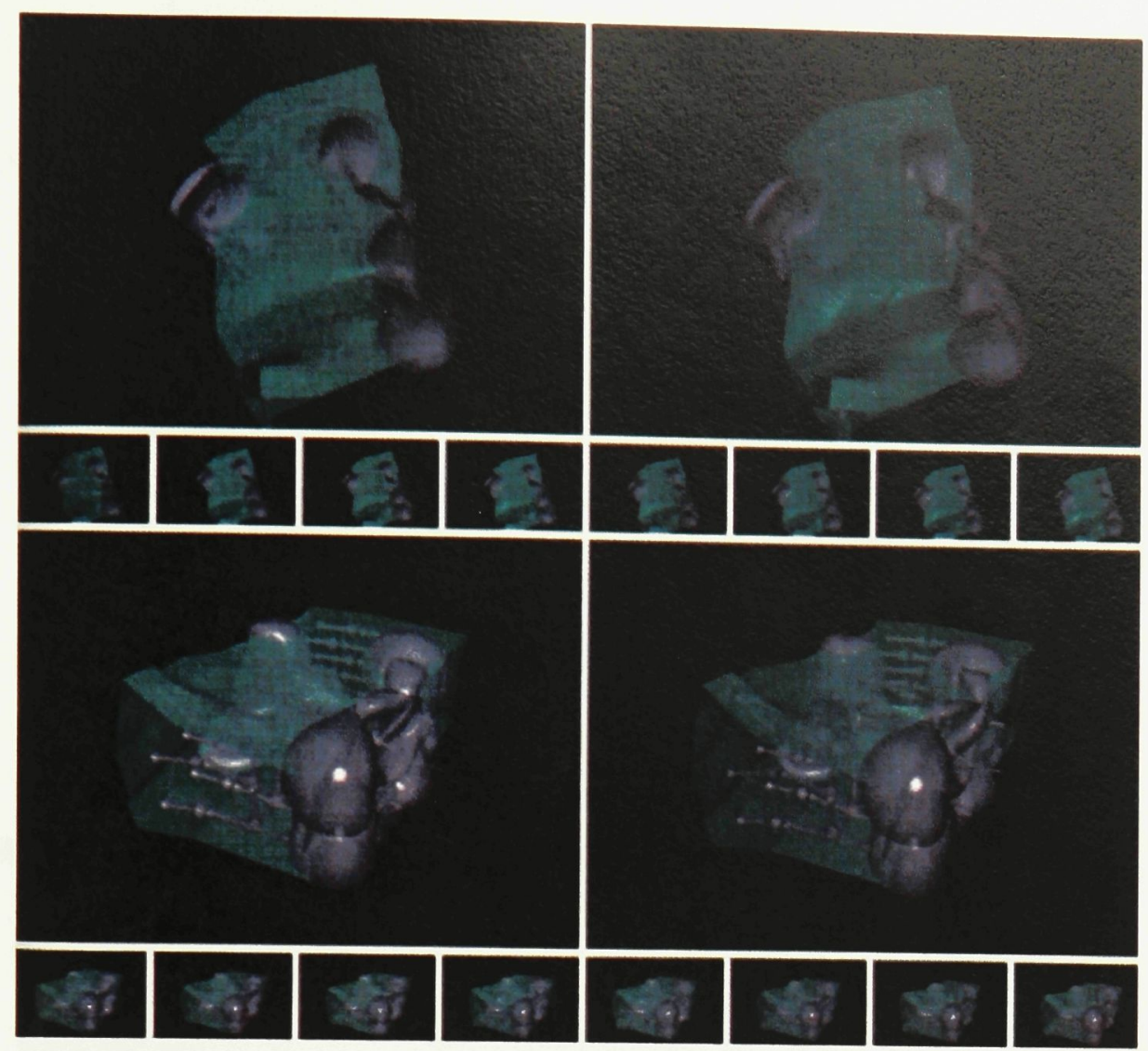

Fig. 1 House Prototype in Long Island. From: Greg Lynn, Animate Form (New York: Princeton Architectural Press, 1999) 153.

shapes and configurations." 25 The design is then generated through the insertion of various "prototypes" into the field of forces, a process that deforms the prototype based on their influences. The final form of the house carries with it the traces of this process; the result is a series of organic forms intertwined three-dimensionally, yet retaining the traces of their
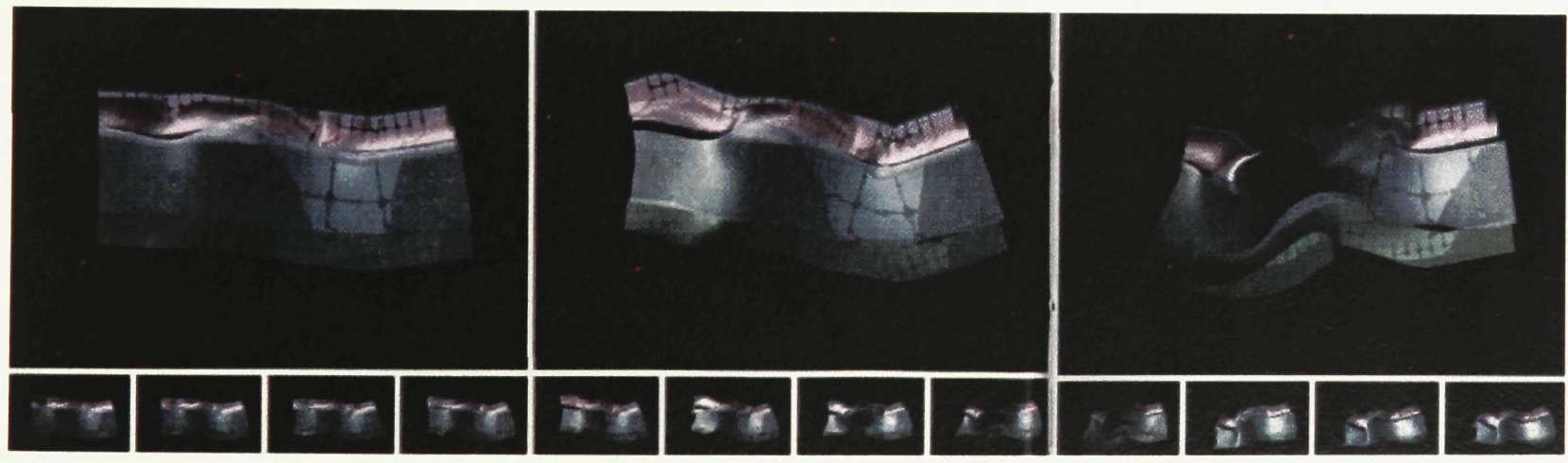

Fig. 2 House Prototype in Long Island. From: Lynn, Animate Form, 160-161.

${ }^{25}$ Lynn, Animate Form, 143. 
transformation from primitive geometric volumes.

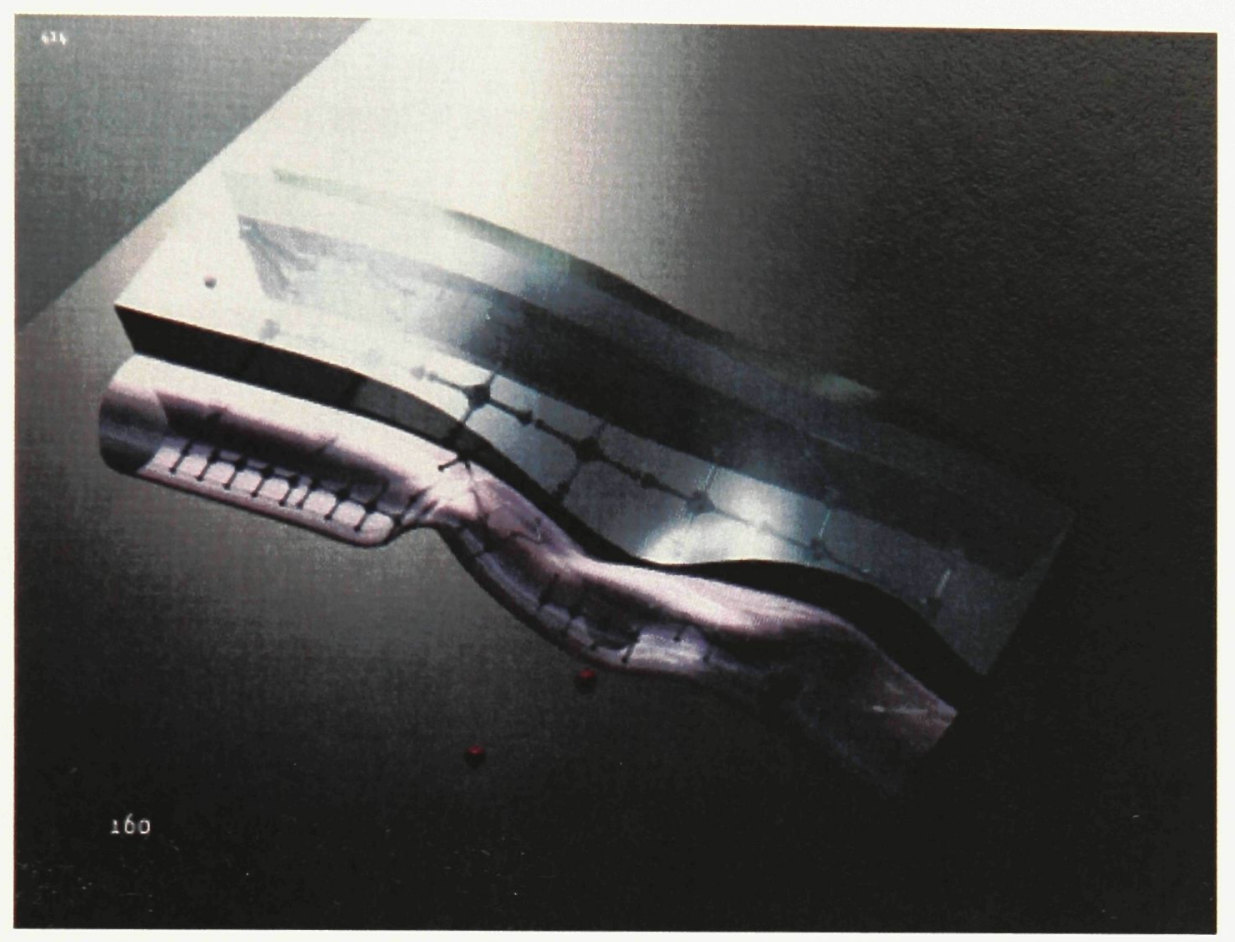

Fig. 3 House Prototype for Long Island. From: Greg Lynn, Animate Form (New York: Princeton Architectural Press, 1999) 160.

While Lynn's process challenges the accepted use of computer technology for architectural purposes, the architecture that results denies the viewer access on a number of levels. The complete visual destruction of the site, as a result of its abstraction into a field of influences, leaves the final form without reference back to its physical site. Furthermore, the house is presented entirely through a series of images that exaggerate its object-ness with exterior views, while denying the viewer any sense of interiority. The result of these two primary factors is an abstraction of an architectural idea that excludes the viewer through the absence of scale, site, or other points of reference. Too much privilege is given to the formal mutation 
of the prototypes; indeed, any spatial sensibility is overshadowed by the software's dominance of the process.

Lynn's project does, however, reveal a key strength of the digital tool: the capacity for animation. While in his "House Prototype" Lynn applies animation to the tectonic transformation of form, an animation of viewpoint is avoided. The former gives a sense of life to the object or space of the model; the latter provides an access for the viewer. The representation of perspectival space in time, through the technique of an animated viewpoint, closely embodies our human relationship to the physical world. At each moment, we perceive our mobile reality visually, in perspective. This sequence of images captured by our eyes carries us through space, defining our experience continuously. Similarly, the animation of a viewpoint in cyberspace carries the viewer through space, facilitating projected inhabitation. Thus we discover the relationship of animation to Bergson's theory of duration, which combines time and memory in order to create experience. Animation in computer modeling technology allows us access to digital space on an experiential level, to a greater degree than in many traditional media. The model becomes a vehicle for animation: when played, this animation presents a visual concept in a manner sympathetic to our own transforming perception of the world.

Although its re-creation is impossible, the representation of elements of the real in cyberspace may augment the experiential relationship of the viewer to the digital model. The complete abstraction in Lynn's "House Prototype" results in a form without reference or meaning. 
Nothing in the representation allows the viewer to draw upon memory in order to relate to the given image. In cyberspace, the inclusion of representations grounded in reality allows the viewer to make associations in memory to past experiences. This in turn provides one with a cornerstone on which to construct a new changing experience, synthesized from associated memories and one's present perception of animated digital space. 


\section{Real: Possible: Virtual}

The reconditioning of our perception through technological advancement suggests a causal relationship of our present and past conditions; our current state is declared to be the result (effect) of former moments (causes). This suggestion is the direct result of scientific advancement in the early part of the nineteenth century, when geometricians and mathematicians sought empirical explanations for all things formerly attributed to the existence of a higher being, or God. In his critique of modern science, Alberto PerezGomez discusses the work of these early scientists: "...the character of objective reason [was described] like this: The present state of the universe should be conceived as the effect of its previous state and as the cause of its future one." 26 This broad theory was quite simply the extrapolation of scientific observations on a smaller scale. For example, at their most basic level, scientific chemical equations begin with a given set of reagents, and end with a predictable set of results: first a cause, then an effect. This observable scientific relationship

\footnotetext{
${ }^{26}$ Alberto Perez-Gomez, Architecture and the Crisis of Modern Science (Cambridge: The MIT Press, 1983)
} 273. 
suggests that the building blocks for any future invention exist in the present, which in turn suggests that these inventions, although not yet conceived, are possible. Any future innovation, once realized, will be declared to have been possible long before its actualization ever occurs.

This empirical view of the relationship of cause and effect soon came under heavy criticism in the work of several philosophers, such as Friedrich Nietzsche and Deleuze. They observed that the declaration of possibility was in fact only provable after the act of creation occurred. In his theory of false causality, Nietzsche describes the act of imbuing past actions with a false understanding of how they came about. He writes: “....all that happened was considered a doing, the effect of a will; the world became to it a multiplicity of doers; a doer (a "subject") was slipped under all that happened." ${ }^{27}$ He realized that the accepted empirical methodology retroactively inserted a defined intention in front of every result, therefore claiming that all innovation was in fact always possible. Deleuze continues this thread in his analysis of Bergson's work, inverting the relationship of the possible to the real:

If the real is said to resemble the possible, is this not in fact because the real was expected to come about by its own means, to project backwards a fictitious image of it, and to claim that it was possible at any time before it happened? ${ }^{28}$

Deleuze's distillation of these terms, namely, the "real" and the "possible", provides an insight

\footnotetext{
${ }_{27}$ Friedrich Nietzsche, "Twilight of the Idols", The Portable Nietzsche, trans. Walter Kaufman (New York: Viking Penguin Inc., 1954) 495.

${ }^{28}$ Deleuze, Bergsonism, trans. H. Tomlinson and B. Habberjam (UrZone Inc., 1988) 97-98.
} 
into their counterintuitive relationship; he in fact suggests that it is not the possible that determines the real, but the real that begets the possible. ${ }^{29}$

Thus we arrive at an understanding of the limitations of the idea of the "possible", which necessarily follows the actualization of a thing and does not precede it. What we often mistake for the realization of possibility is in fact the actualization of the virtual. The virtual, as Eric Alliez interprets it from Bergson and Deleuze ${ }^{30}$, is described thus: "the virtual is not actual; rather it possesses an ontological reality as such that denies and exceeds any logic of the possible." 31 The virtual, born from some combination of elements of the real and the memory of the viewer, exists in a moment of transition in which the viewer experiences an extension of his present state. This extension is demonstrated in the earlier example of the viewer's synthesis of experience from digitally represented space. The model presents the viewer with an image of space that transforms through animation; the space, however, does not exist, except on the two dimensional surface of the monitor. However, by projecting oneself into the space of the digital model, one is able to capture a sense of experience generated from one's perception of the space and the application of memory. This is a condition of the virtual; the viewer's perception is extended through the representation of space, resulting in an experience without empirical explanation. This extension is only momentary, for it requires a mental dissociation from understanding, and exists only for as long as the analysis of the virtual condition is resisted. Since analysis functions on immobile

${ }^{29}$ Deleuze, Bergsonism, 97-98.

30 In his discussion of Bergson, Deleuze borrows the following definition to clarify his idea of the virtual: "Here again Proust's formula best describes the states of virtuality: 'real without being actual, ideal without being abstract.'" Deleuze, Bergsonsim, 96.

31 Eric Alliez, "The Virtual Deleuze", ANY (no. 19-20, 1997) 19.8. 
past moments, this temporary virtual condition occurs in the present; thus we associate the virtual to duration, placing it in the realm of intuition, where it relies on the singular moment of the present to transport the viewer beyond themselves into a space of projected inhabitation.

The virtual in architecture, whether we are speaking of built or unbuilt projects, is closely linked to experience, both of which are fleeting and momentary. The digital medium has the capacity to elicit an experiential reaction from the viewer, thereby revealing a virtual condition in digital space. The apprehension of this virtual condition by the viewer is its actualization. Once the viewer perceives it, the virtual becomes possible through this actualization in the viewer's mind. 


\section{Animate Experience}

The advancement of digital technology has provided architects with a tool for spatial representation in the form of cyberspace. While the "space" of cyberspace is often referred to as " $3 \mathrm{D}$ space", it is in fact restricted to the two dimensional plane of the computer screen. Because of this, cyberspace relies on many of the same traditions as drawing and photography to convey ideas of space, most significantly the tradition of perspective. In all of these media, a similarity in representation to the human-eye perception of the world provides a mental link for the viewer, allowing one to project oneself into the represented space of the image. This projection is very much an exercise in virtuality, for the viewer must suspend their understanding of the image as a two dimensional plane in order to complete their projection of self into a space of imagination. This process is extremely subjective, as it relies on the memory of the viewer to construct an experience of space from the given representation. Kant reveals this dependence on memory in his work Critique of Pure Reason: 
But even the presentations of space and time are mere schemata related always to reproductive imagination which summons the objects of experience. Without objects they would have no meaning. And so with all concepts. ${ }^{32}$

Indeed, the "reproductive imagination" to which Kant refers is memory, which draws on past experience to make sense of the present moment. Without memory, perception becomes meaningless! Thus, it is the viewer's specific past experiences that condition the projection of self into the space of the image. The architect must invent modes of representation that tap into this mental projection to the fullest degree, allowing for subjective penetration and the actualization of virtualities.

The technology of cyberspace offers new opportunities for architectural investigation through representation which allow for the communication of experience in a manner that is sympathetic to human perception. This is particularly true of animation in cyberspace, which combines the tradition of the perspectival image with the element of time. The human mind has an intuitive ability to comprehend the relationships of objects in perspectival space when they unfold in time, since this is in fact the manner in which we comprehend our everyday environment. Therein lies the greatest potential for the digital medium: it provides the architect with a powerful way to communicate human experience in a time-based form. With great optimism, Schawn Jasmann writes about this potential:

32 Kant, Critique of Pure Reason, 66. 
This is precisely the condition in which new architectural possibilities exist. Hence, this space of perceptual transformation brings to fore new experiential realms... Architect-designers thus become perceptual painters of animate experience through a technological medium that permits the sensorial intensity of an experience of movement, color, form, and space. ${ }^{33}$

In order to reveal the advantages of the digital medium that permit the creation of this "animate experience", we may consider McLuhan's four Laws of Media. Each Law, phrased in this instance as a question, targets a unique facet of the new technology in an effort to discover the fundamental qualities that affect our perception as well as our use of the tool.

Law of Media \#1: "What does the artefact enhance or intensify... or accelerate?”34

McLuhan describes the new technology as artefact, which in the present discussion refers to the technology of cyberspace. This fist Law addresses the advantages of the medium, which fall into three categories: acceleration, simulation, and animation.

\section{Acceleration}

The digital medium allows for a significant increase in the speed of production of architectural representations. A single model may yield hundreds of images. This is a direct evolution of the more traditional medium of drawing which, as will be discussed

\footnotetext{
33 Schawn Jasmann, "Virtual Architecture and the Role of Inscription", Hybrid Reality: Art, Technology and the Human Factor, ed. Hal Thwaites (Montreal, Canada: International Society on Virtual Systems and Multimedia, 2003) 422.

${ }^{34}$ Eric McLuhan and Marshall McLuhan, Laws of Media (Toronto: University of Toronto Press, 1988) 98-99.
} 
shortly, is not replaced by the new technology, but absorbed and retrieved in a new form. This acceleration of production also applies to the construction of physical models, as the technology of rapid prototyping continues to advance. Using digital models, architects are able to evaluate a greater number of possibilities in a shorter amount of time.

\section{Simulation}

As discussed earlier, simulations cannot aspire to the true complexity of the real, yet computer modeling technology is able to approximate certain conditions more accurately than the traditional media of drawing and modeling. The most significant of these conditions is the calculation of light: digital technology is able to create and manipulate light sources and behaviors in many situations that elude more traditional modes of representation. An interior scene, for example, may contain multiple light sources, whose effects, in a traditional drawing, must be approximated through the intuitive ability of the artist. In the digital medium, materiality and lighting variables allow the architect to discover nuances in reflection and refraction that may easily be overlooked otherwise. The power of the tool for calculating sun angles and intensities also provides the architect with a significant insight into site conditions at different times throughout the year. In both of these situations, the capacity of the computer to perform complex calculations allows the architect to manipulate the computer model and quickly realize the changes in lighting that occur due to the 
modifications.

\section{Animation}

One of the roles of architecture in the built environment is to evoke emotion and manifest meaningful experiences for those in its presence; Jasmann's definition of “architect-designers as stagers of human experience" 35 is fitting in that it captures the seductive, artistic vein of the discipline that inspires much of architectural education. Animation is the face of digital technology that has the most significant contribution to make to the art of architecture. Through animation, the architect is able to capture an experience of space that unfolds in time. By infusing the perspectival image with the dimension of time, the animated architectural representation satisfies the viewer's sense of duration. It is this quality of animated cyberspace that leads Grosz to inscribe it as the "space of mental inhabitation"36, suggesting that this format of representation is indeed the psychological sibling to our physical inhabitation of the real.

Law of Media \#2: "If some aspect of a situation is enlarged or enhanced, simultaneously the old condition or unenhanced situation is displaced thereby. What is pushed aside or obsolesced by the new organ?" 37

\footnotetext{
35 Jasmann, "Virtual Architecture and the Role of Inscription", 422.

${ }^{36}$ Grosz, Architecture from the Outside, 88.

${ }^{37}$ McLuhan, Laws of Media, 99.
} 
As new technologies evolve and alter our sphere of perception, they inevitably displace existing techniques and methodologies. Digital technology, with its ability to accelerate, simulate, and animate architectural representation, has already begun to obsolesce the analog techniques of drawing and modeling. This is not to say that these traditional methods of working have lost their value; McLuhan offers this clarification of his terminology: "Obsolescence is not the end of anything; it's the beginning of aesthetics, the cradle of taste, of art, of eloquence and of slang." ${ }^{38}$ Thus we understand obsolescence to suggest that the process of design is challenged and ultimately improved through newer techniques and media, while traditional modes of working maintain their (diminished) viability.

A consideration of how the two primary analog techniques of drawing and modeling have been obsolesced by cyberspace reveals the enhanced qualities of the digital medium and clarifies the distinction between an obsolescence that replaces versus an obsolescence that augments through differentiation.

\section{Drawing}

The conventions of architectural drawing, such as plan, section, elevation, and perspective are part of the vocabulary through which a building's details are developed and communicated. Digital modeling technology employs these standards as a system of coordinates for a

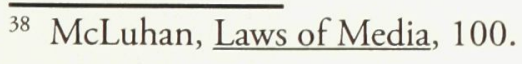


limitless digital space. In addition, texture mapping and lighting in digital space begins to obsolesce rendering by hand as a method for communicating spatial and material characteristics. The aspects of drawing that are enhanced in digital technology are specifically in three categories: acceleration (the capacity for a great volume of production in a short time), simulation (an improved approximation of the conditions of the real), and animation (a representation of architectural space in time).

Acceleration: Digital modeling allows the architect to develop a design in three dimensions; as a result, a single model contains plans, sections, elevations, and perspective views simultaneously. Any change made to the design is immediately reflected in all of these conventional representations. The analog method of drawing requires changes in plan, section and elevation to be resolved individually, whereas the digital method is able to use a single model as a generator for a wide array of images quickly. This acceleration of production obsolesces analog drawing in terms of raw quantity; perhaps more importantly, however, is that this new technology permits the architect to explore a multiplicity of concepts rapidly.

Simulation: The simulation capabilities of the digital medium offer a greater degree of accuracy than drawing for those interested in the practical reality of constructed architecture. This does not only apply to the technology and execution of construction proper, but also to the constructed existence of the building and its predicted spatial qualities. While 
the medium of drawing allows for incredible flexibility in the representation of intended realities, the complex effects of light and materiality in space are necessarily simplified by the imagination and knowledge of the architect-artist. Evans observes this while discussing the installations of James Turrell:

The drawing has intrinsic limitations of reference. Not all things architectural (and Turrell's rooms are surely architectural) can be arrived at through drawing. There must also be a penumbra of qualities that might only be seen darkly and with great difficulty through it. ${ }^{39}$

In digital space, the computer is able to extrapolate the reflective, ambient qualities of light/material interactions, providing the architect with a visual representation of spatial quality that more closely approximates its eventual reality. ${ }^{40}$ Proponents of analog drawing often romanticize the discrepancy between the imagined representation of a space on paper and its physical manifestation, referring to unpredicted events as "happy accidents". While many may suggest that the accuracy of simulation in the digital model limits the imaginative freedom of the designer, few realize that it is in fact a very subtle yet powerful shift that has taken place. The "happy accident" is now allowed to happen before construction, in cyberspace, where its apprehension allows the architect to emphasize its beauty in the final scheme.

\footnotetext{
39 Evans,"Translations from Drawing to Building", 159.

40 The creation of accurate lighting simulations requires a certain degree of technical proficiency with the software. While an inexperienced user may often generate inaccurate approximations, the software is inherently capable of producing results that very closely approximate physical reality. A certain level of user proficiency is assumed in order to proceed with the discussion.
} 
Animation: The medium of drawing is immobile; while motion may be indicated through drawing, actual motion is impossible in a single image due to its static existence. In order to introduce the aspect of time into drawing, techniques were invented that required the participation of the viewer in order to create a sense of the passage of time. The graphic novel is the drawing technique that most closely relates to animation; indeed, in the production of movies, a storyboard often precedes the moving images of the film. The graphic novel combines the elements of spatial representation and narrative, which in turn suggests a dimension of time. The passage of time in a graphic novel seeks to involve the memory of the viewer, in essence expanding the virtual world of the novel through the implication of aspects of duration. Because the element of time is fragmented in the graphic novel, an exercise of projection and extrapolation is required on the part of the viewer in order to access the experiences of the narrative. In the digital medium, this extrapolation is negated, and the projection facilitated, by the sympathetic relationship of the animation to the viewer's sense of time. The passage of time in the animation, while it plays in front of one's eyes, is parallel to one's own passage of time; the animation becomes a set of "movement-images which are mobile sections of duration" ${ }^{\prime 1}$. The alignment of image and duration draws the viewer more readily into the experience of the animated spatial condition.

\section{Modeling}

Architectural scale modeling is often practiced alongside drawing, since it allows the designer

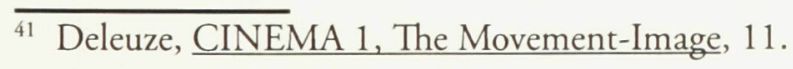


to visualize space and form in three dimensions. Digital modeling, as the name suggests, is most directly an evolution of this technique. The $\mathrm{XYZ}$ axis assignment in digital space allows the user to conceive of the three dimensions of width, depth and height that stem directly from the physical realm. In a very similar method, the forms of the computer model and the physical model are combined considering all three dimensions. There are, however, still significant differences between the two that attest to the obsolescence of the analog by the digital.

Acceleration: The acceleration potential of the computer model is most evident in terms of its flexibility. Since there is a great similarity between the two modeling techniques in terms of the production of images (a single physical model can yield hundreds of photographic images, much the same way a digital model may be rendered from hundreds of viewpoints), the acceleration potential of the digital model resides in its variability. The facility with which a digital model can transform far surpasses the inherent immobility of its physical alternate. This is not to say that a physical model is a fixed entity; there are, however, certain aspects of a computer model - materiality, for instance - that can be continuously altered and adjusted. Since the experience of architectural space is conditioned as much through materiality as through form, this flexibility allows the architect to investigate several different virtual conditions rapidly.

Simulation: In terms of simulation, the obsolescence of the physical model occurs in the flexibility of scale offered by the digital model. In a physical model, the effect of light on its 
spatial quality is greatly affected by the scale of the materials used in its construction. Physical materials are required to assume a disguised identity in order to introduce a new scale into the model. Furthermore, multiple models are often required in order to investigate various levels of detail. One is often required to make associations between these different models in order to understand the effects of detail on the greater spatial composition. Because a digital model is infinitely flexible in its scale, a single model may contain fine detail as well as a wider spatial representation, facilitating an appreciation of their interconnectedness. In terms of materiality, the materials of a digital model exist at a one to one scale: they need not be disguised in order to achieve their desired effect. A single digital model can therefore succeed at multiple scales simultaneously, allowing for a more unified perception of the project as whole.

Animation: The issue of scale also presents itself as the discussion turns to animation. Animation, in the form of video, is possible using a physical model. One can create an animated viewpoint that facilitates the viewer's access to the space of the model. However, because the model is a scaled representation of the larger intended architectural reality, the act of projection required by the viewer is more involved; the viewer must suspend the recognition of the model as a one to one object in order to be immersed in its scaled, represented space. The digital model bridges the gap of this dual existence; the architectural model in cyberspace is not a scaled version of a lager spatial concept. Because of this, the act of projected inhabitation through animation is enhanced in the digital format; the scale is 
set only by the terms of reference of the model itself, allowing the viewer to relate directly to the digital model on a one to one level. This relationship facilitates the viewer's access to the virtual condition of the digital model when the time of the animation enters one's own duration.

Law of Media \#3: "What recurrence or retrieval of earlier actions and services is brought into play simultaneously by the new form? What older, previously obsolesced ground is brought back and inheres in the new form?" 42

Once an understanding of the obsolescence of previous media is understood, we must consider McLuhan's third Law of Media. The third Law reveals qualities in the new media that are directly recovered or carried forward from past techniques.

From drawing, the digital tool has carried forward the standards of orthographically projected representations, such as plan and section. These views of space are used simultaneously in digital modeling to compose elements in space. Many digital modeling softwares ${ }^{43}$ operate in a quadrant layout, with three of the four views presenting the orthographic projections of top (plan), front (elevation) and side (section). The fourth view, in a standard layout, provides a perspectival vantage point from which to observe the results of changes made in the various other viewports. Of course, this default interface is not necessarily the layout of choice for many users; it does, however, reveal the direct recovery of

\footnotetext{
42 McLuhan, Laws of Media, 99.

${ }_{43}$ Maya, Form Z, 3ds MAX.
} 
standard representations developed through the medium of drawing. Once recognized, we are able to challenge the digital medium by altering this given interface, allowing the user more or less access to the full breadth of information provided. For example, by choosing to develop an architectural concept through perspective alone, the architect is able to project his own inhabitation as he works, while simultaneously generating the accepted orthographic representations along the way. This link to tradition, in terms of the legibility of standard architectural drawings, provides a point of comparison between old and new methodologies that may reveal the unique qualities of each.

From physical architectural modeling the digital tool retains a necessary link to photography. In a physical model, photography is very often the filter through which the architect captures and controls a vision of space. This technique presents a viewpoint grounded in perspective and often transforms the scale to allow the viewer to suspend belief and place themself in the model through projection. The denotative quality of the photograph permits this transformation to occur in the mind of the viewer; Roland Barthes observes this quality in his writing:

In order to more from the reality to its photograph it is in no way necessary to divide up this reality into units and to constitute these units as signs, substantially different from the object they communicate...Certainly the image is not the reality but at least it is its perfect analogon and it is exactly this analogical perfection which, to common sense, defines the photograph. ${ }^{44}$

$\overline{44}$ Roland Barthes, "The Photographic Message", A Barthes Reader, ed. Susan Sontag (New York: Hill and Wang, 1982) 196. 
The tendency of the viewer to associate the photographic medium to an existing reality allows photographs of a physical model to disguise its true scale. The viewer requires this mediation in order to access the space of the model. In much the same way, our only access to the spaces of a digital model is through the lens of a digital camera; this lens most often presents itself as the plane of the computer screen. It is through the manipulation of this digitally simulated camera that cyberspace is presented to the user. Indeed, as soon as the camera is removed, digital space ceases to exist. This temporary existence of digital space emphasizes its virtual condition; the model exists either in relationship to a viewer, or not at all.

Law of Media \#4: "What is the reversal potential of the new form?" 45

Reversal occurs at the point at which, when pushed, a medium takes on characteristics that are somehow oppositional to its original character. McLuhan offers examples of this effect:

Money (hardware), pushed to its limit, reverses into lack of money, that is, credit (software or information), and the credit card. At high speed or in great quantity, the motor car reverts to its nautical form, and traffic (or a crowd) 'flows'. ${ }^{46}$

McLuhan suggests that this reversal is a tendency of all media; furthermore, it is neither by necessity a positive or a negative occurrence. We may hypothesize as to the potential for

\footnotetext{
45 McLuhan, Laws of Media, 99.

${ }^{46}$ McLuhan, Laws of Media, 107.
} 
reversal in a given medium; it can only be fully recognized, however, once it has taken place. Thus the observation of the reversal makes it possible in retrospect.

The reversal of the digital medium has occurred in the development of three-dimensional printing and stereolithographic technology. This technology translates the virtual forms of the digital model into a scaled, physical, real object. Thus a reversion to physical modeling takes place - while this process allows the new physical model to contain complex and accurate geometry, it once again relies on photography in order to communicate ideas of space and

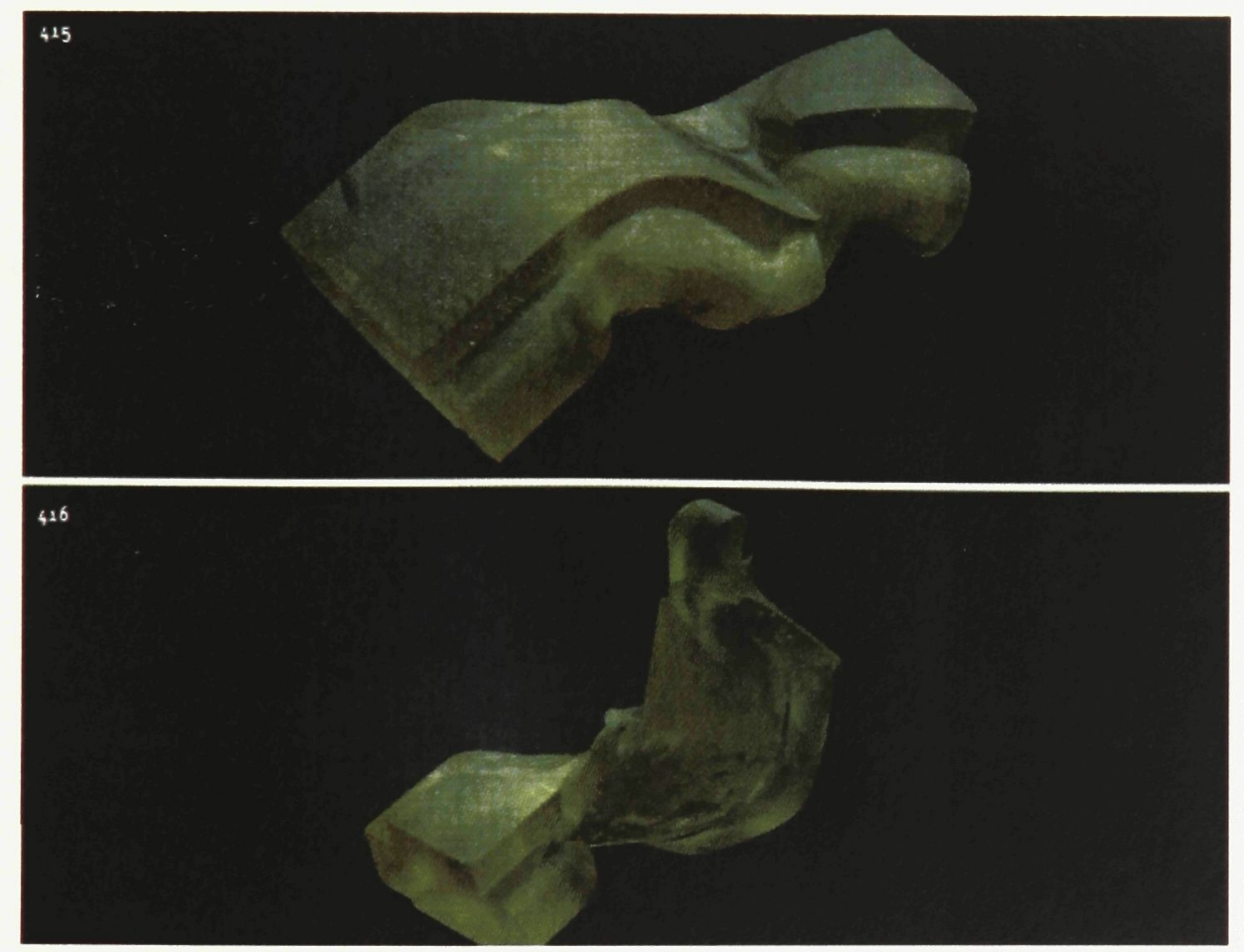

Fig. 4 Stereolithography model of House Prototype in Long Island. From: Lynn, Animate Form, 161.

inhabitation. The enhanced qualities of materiality and scale that the digital model achieves 
resist this translation: because of this, the physical scaled reduction of the digital model does not intensify the synthesis of projected inhabitation. The utility of this reversal, then, applies more to a process of clarification than exploration. It renders explicit the tectonic structure of a design, to the exclusion of all other conditions.

Since we are presently concerned with the process of design and its relationship to experience and duration, this reduction through reversal must be resisted. Indeed, such a formal translation of the spaces of the digital model threatens to leave behind the qualities of experience and duration that are enhanced by this medium. The translation to architectural form of these experiential conditions should involve the architect rather than exclude him; indeed, the subjective nature of the invented experiences requires subjective involvement in their translation. 


\section{Translation}

There must exist at some point in the process of design a moment of translation, where the experiential nature of the concept is transformed into a language of construction. While still in the realm of representation, this translation is the point of the actualization of the virtual. Once the desired experiential condition is represented in animated digital space, the architect must translate the essential elements from this virtual condition and assemble their synonymous alternate into a language of construction. It is at this point that the architectural project transforms from a purely conceptual, experiential representation into realizable, buildable form. This transformation necessarily considers the eventual reality of the architecture; while the representation remains digital, it more directly simulates the conditions of the real. Thus, the virtual experience is actualized into our minds through this translation, allowing a possible reality to become a real possibility.

The translation of digital expression into buildable form is undertaken briefly in Eisenman's 


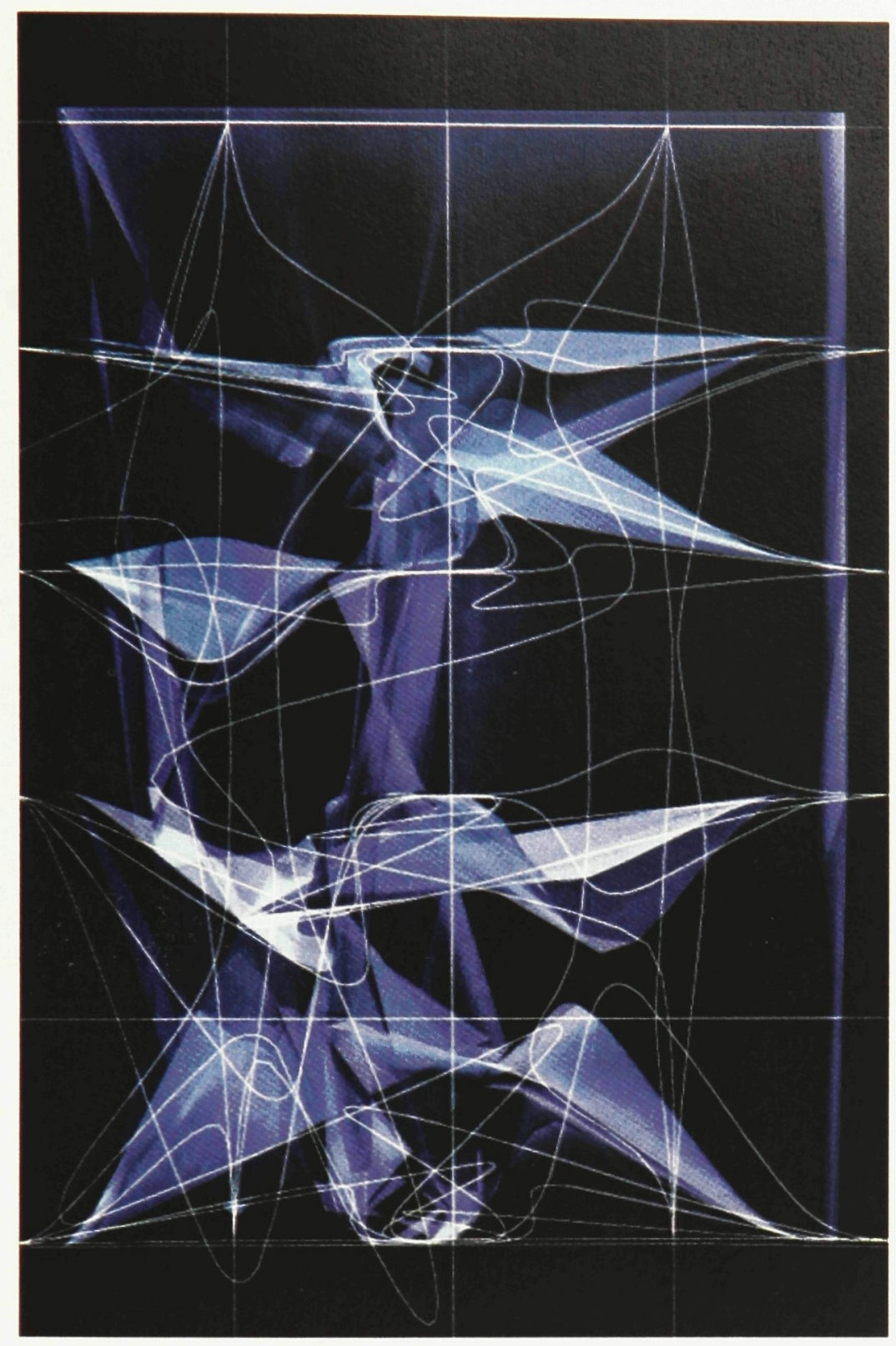

Fig. 5 Eisenman's Virtual House. From: Luca Galofaro, Digital Eisenman: An Office of the Electronic Era, trans. Lucinda Byatt (Boston: Birkhäuser, 1999) 65.

"Virtual House" project. Eisenman's process of deformation, which dictates the final form of the digital model, is subject to much of the same criticism that applies to Lynn's "House Prototype in Long Island"; an absence of site, as well as object-like exteriority, deny the viewer's attempts to project inhabitation. However, Eisenman takes this project a step further 

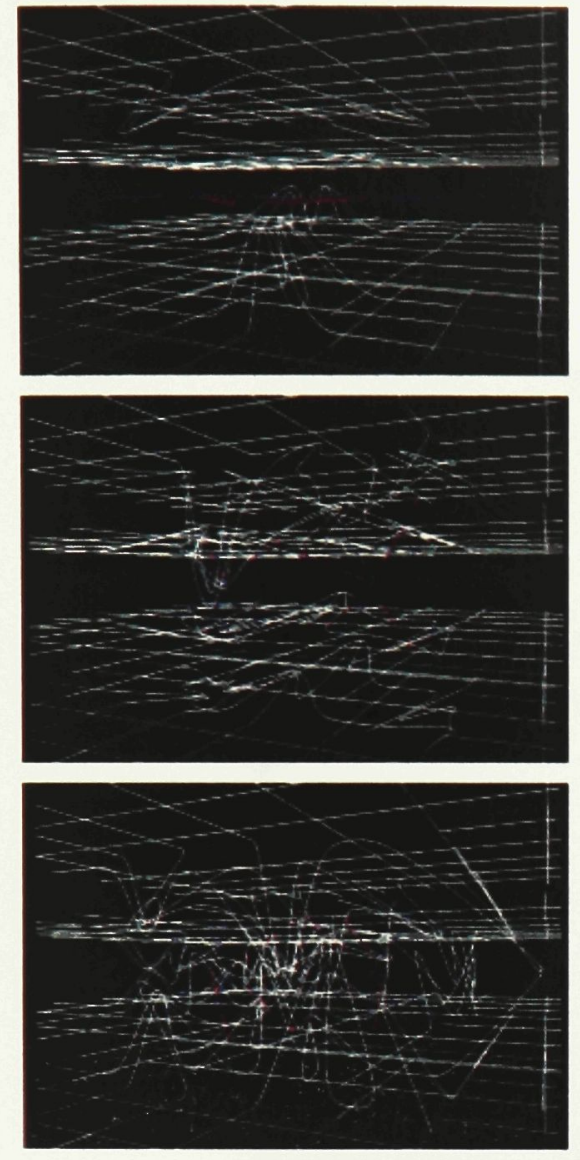

Fig. 6 Genesis of the Virtual House. From: Galofaro, Digital Eisenman: An Office of the Electronic Era, 61.
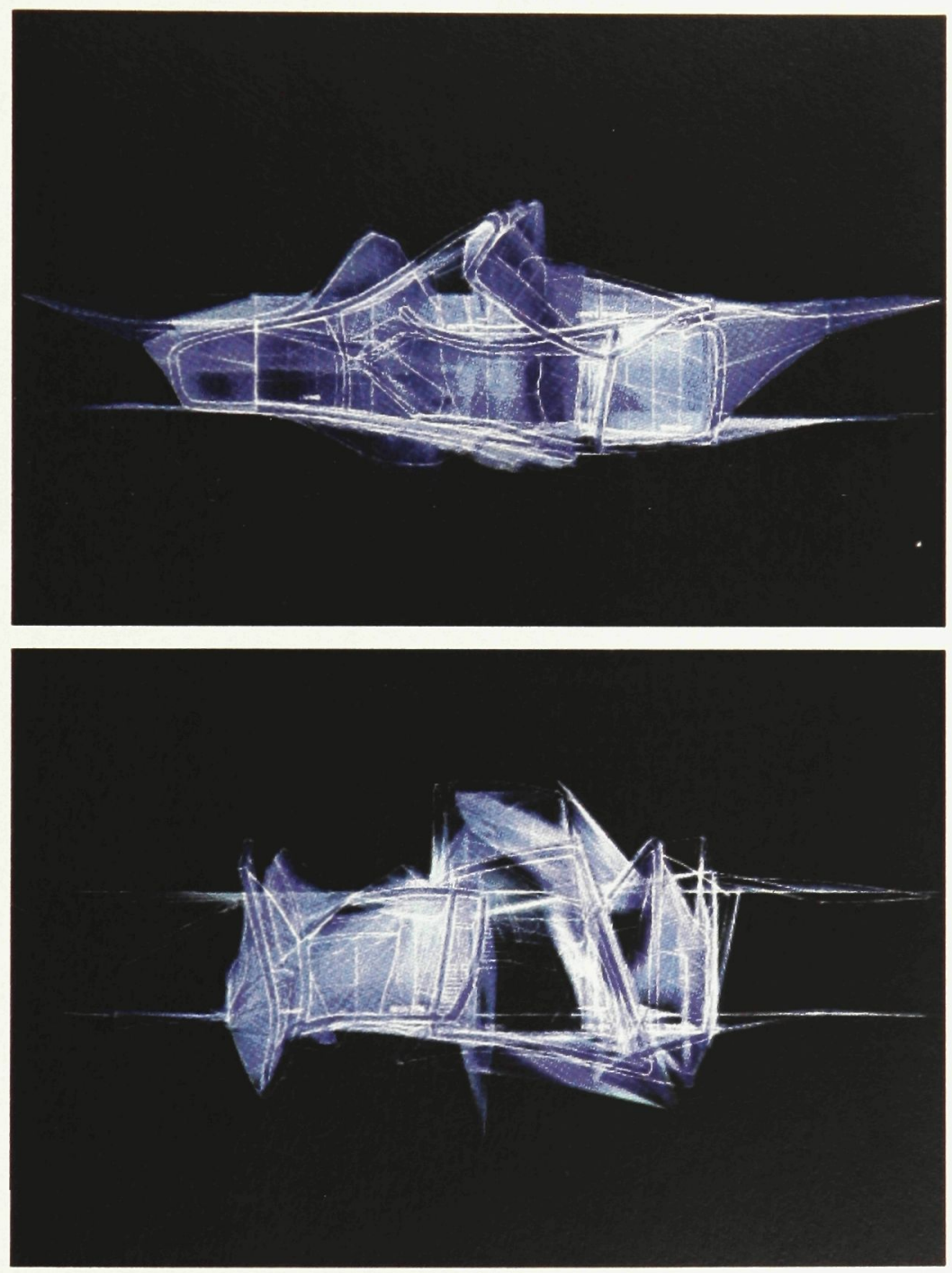

Fig. 7 Sections of Eiseman's Virtual House. From: Galofaro, Digital Eisenman: An Office of the Electronic Era, 68-69. 
than Lynn by applying program and function to the spaces discovered in the final form.

This is accomplished by tracing orthographic plans and sections over rendered images. This

literal transcription at last provides the viewer with some sense of scale, which to some degree
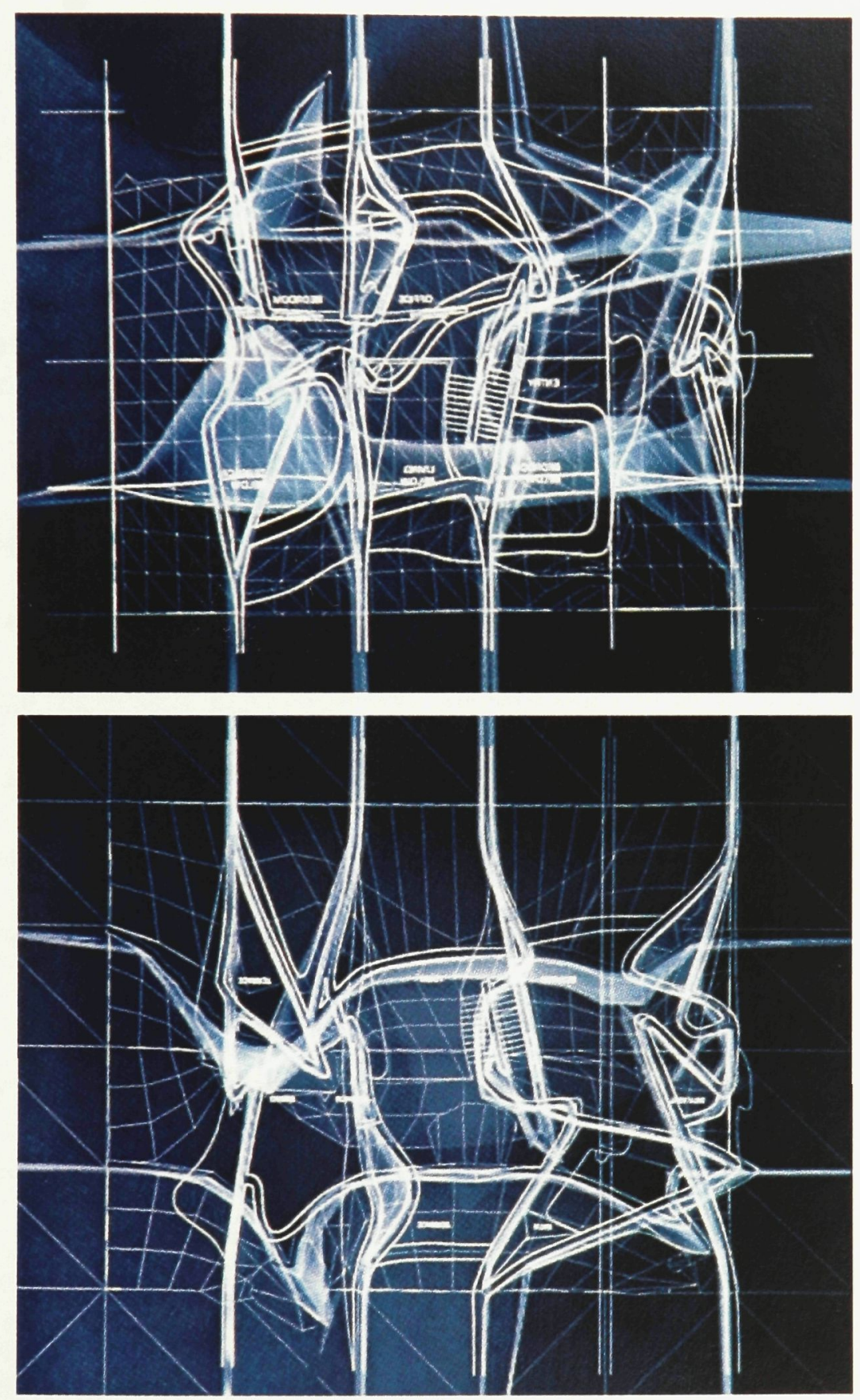

Fig. 8 Plans of Eisenman's Virtual House. From: Galofaro, Digital Eisenman: An Office of the Electronic Era, 68-69. 
assists in the reading of the images that precede them. Still, there is no further suggestion of materiality or the presence of light to convince the viewer of the possible existence of the house in physical space.

Eisenman suggests that the computer model generated for this project resists translation into buildable form, since the medium embodies other priorities:

The electronic paradigm directs a powerful challenge to architecture because it defines reality in terms of media and simulation, it values appearance over existence, what can be seen over what is. ${ }^{47}$

Little emphasis is placed on the realization of this project as built form; the tracing of the plans and sections directly from the generated form is of little importance, and is often excluded from the project in publication. ${ }^{48}$ In resisting a more in depth translation, this project remains purely in the realm of theoretical discussion. To affect the integration of digital technology in modern architectural design, a process that extends beyond this theoretical boundary must be invented.

Zaha Hadid's Lois \& Richard Rosenthal Center for Contemporary Art in Cincinnati, Ohio, embodies different approach to digital technology. Hadid used computer modeling

\footnotetext{
47 Peter Eisenman, "Visions unfolding: architecture in the age of electronic media", Domus (no. 724, 1992) 17.

48 Three primary sources were researched for this project: Peter Eisenman, Diagram Diaries (New York: Universe Publishing, 1999); Luca Galofaro, Digital Eisenman: An Office of the Electronic Era, trans. Lucinda Byatt (Boston: Birkhäuser, 1999); and Ingeborg Rocker, "The Virtual: The Unform in Architecture", ANY (no. 19-20, 1997). Only Galofaro's book included images of the traced plans and sections. Indeed, they were even excluded from Rocker's essay in the Virtual House issue of ANY magazine, where the project originated.
} 
in conjunction with analog media in the development of her scheme; however, unlike the two previous examples, Hadid's project uses computer technology as a vehicle for the communication of concept and spatial condition rather than as a generator of form. An animation that accompanied her competition entry reveals the conceptual transformation of an "Urban Carpet" 49 and its influence on the spatial and programmatic arrangement of the design. The folding forms of the animation begin as representations of abstraction, suggesting certain attitudes towards site and the project itself. As the "Urban Carpet" curls into place, aligning the remaining forms into the recognizable arrangement of the façade, the viewer

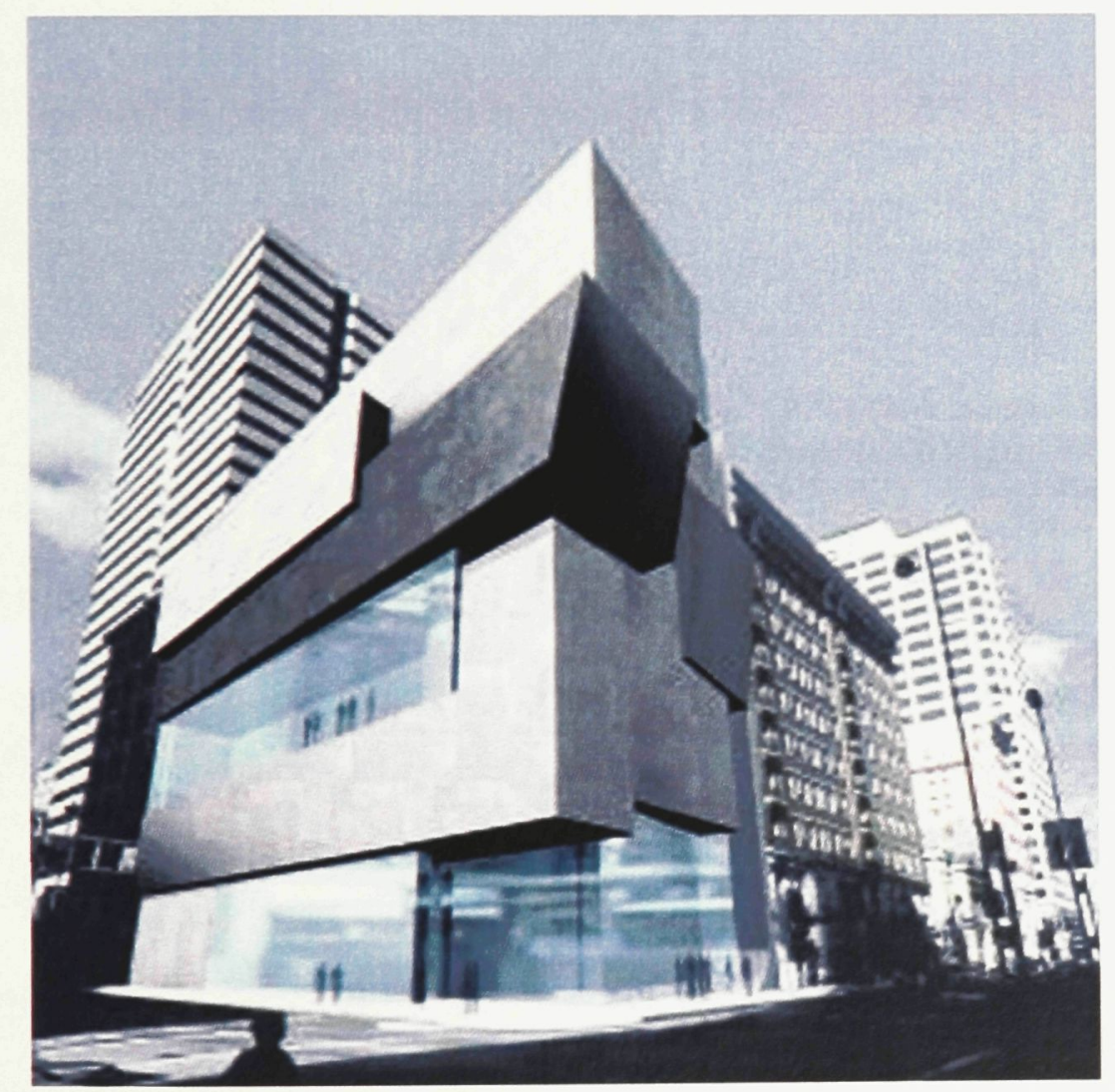

Fig. 9 Digital model of Hadid's Rosenthal Center for Contemporary Art. From: "Building Motion: The Architecture of Zaha Hadid", Place Tower Arts Center - Exhibitions (Sept. 10, 2005) < http://www. pricetower.org/art/Archive/zaha.html>.

\footnotetext{
49 A term used by Hadid to describe the conceptual transformation of the fabric of the horizontal city into a
} vertical expression, one of the projects primary architectural gestures. <http://www.zaha-hadid.com/>. 

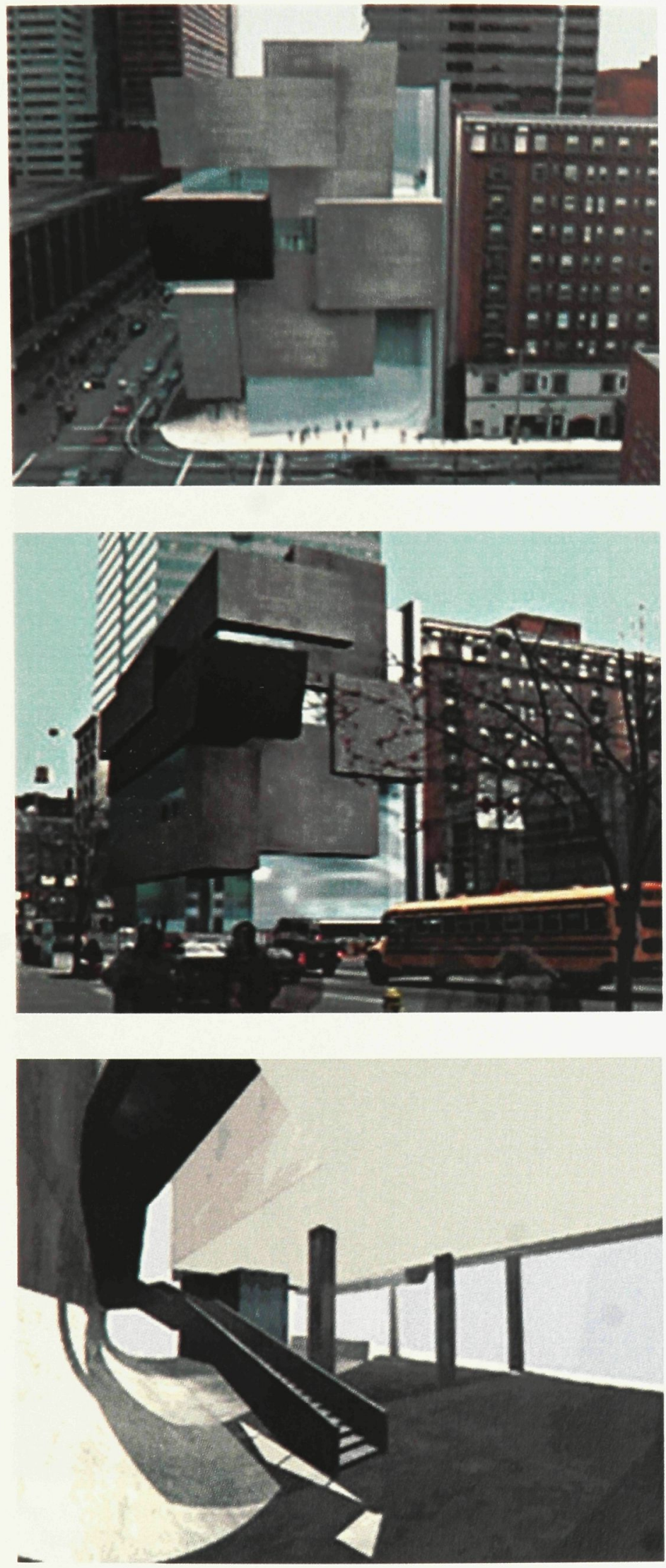

Fig. 10 Digital Model of Hadid's Rosenthal Center for Contemporary Arts. From: "The Lois \& Richard Rosenthal Center for Contemporary Art, Cincinnati", DesignBoom (Sept. 10, 2005) < http://www. designboom.com/portrait/zaha_c.html>.

Fig. 11 Digital Model of Hadid's Rosenthal Center for Contemporary Arts. From: "The Lois \& Richard Rosenthal Center for Contemporary Art, Cincinnati", DesignBoom (Sept. 10, 2005) < http://www. designboom.com/portrait/zaha_c.html>.

Fig. 12 Digital Model of interior of Hadid's Rosenthal Center for Contemporary Arts. From: "The Lois \& Richard Rosenthal Center for Contemporary Art, Cincinnati", DesignBoom (Sept. 10, 2005) < http://www. designboom.com/portrait/zaha_c.html>. 
comes to understand the animated composition as a building. Hadid's images demonstrate a translation from conceptual language to a language of construction; the animation reinforces this translation, revealing the connection between the two.

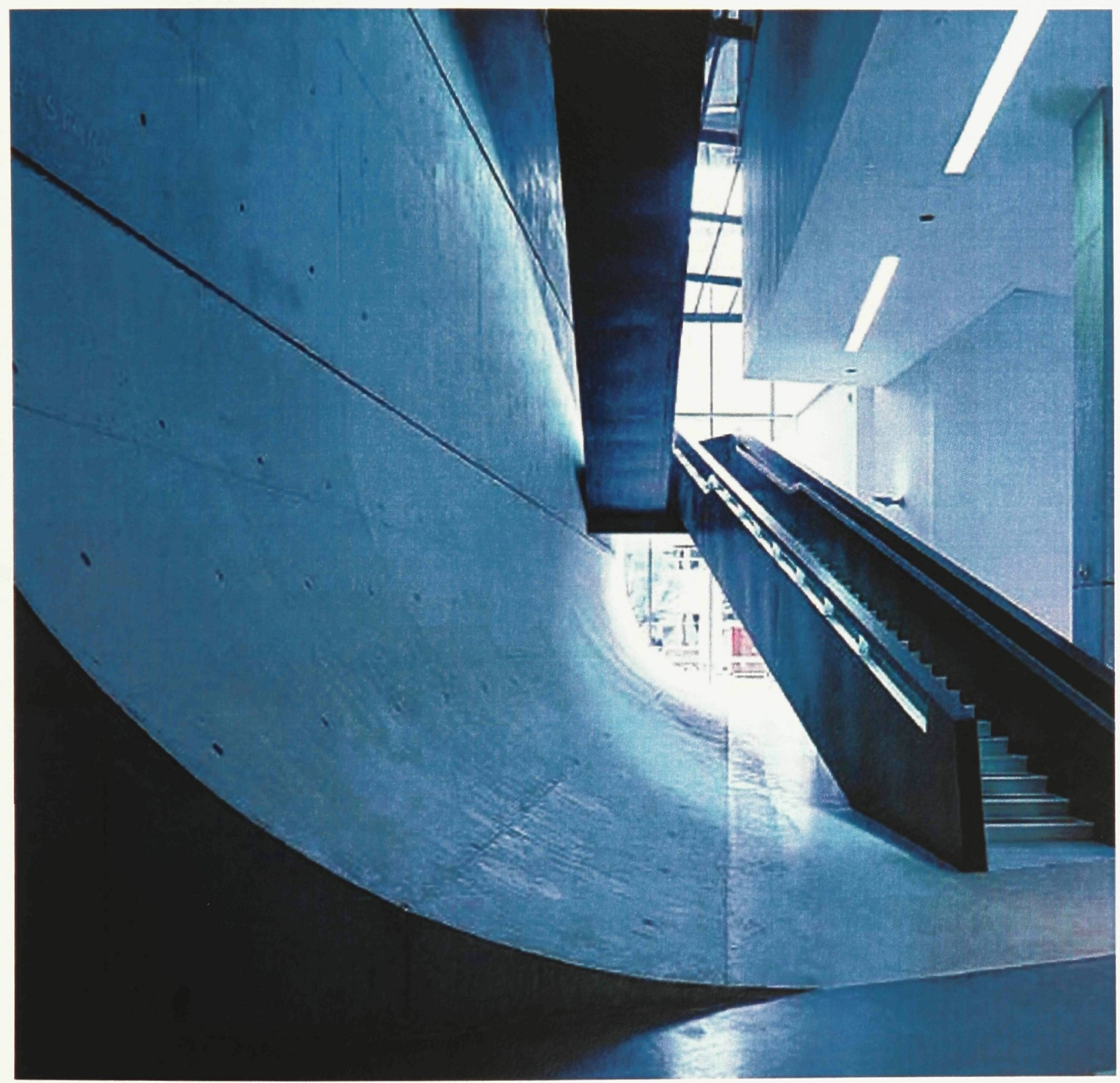

Fig. 13 Interior of Hadid's Rosenthal Center for Contemprary Arts. From: ArcoWeb (Sept. 10, 2005) < http://www.arcoweb.com.br/arquitetura/arquitetura466.asp>. 


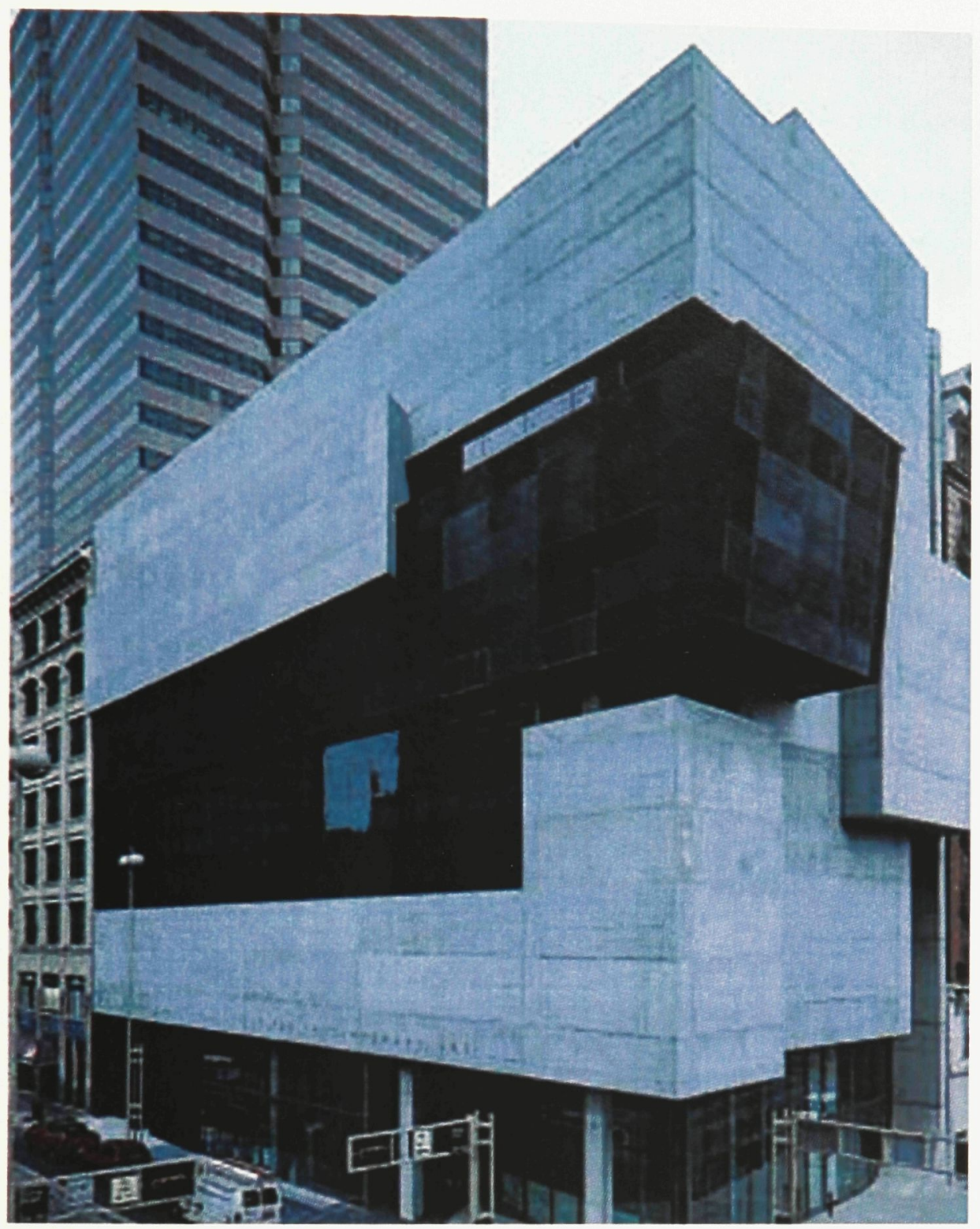

Fig. 14 Hadid's Rosenthal Center for Contemporary Arts, Cincinnati. From: ArcoWeb (Sept. 10, 2005) < http://www.arcoweb.com.br/arquitetura/ arquitetura466.asp>

Because Hadid's animation comes near the end of the design process, it does not explore a mobile virtual experience as an influencing factor in the generation of the architecture.

Nonetheless, the effectiveness of Hadid's translation from concept to built form is a precedent that should be aspired to when considering the realization of a digitally represented experience. 
A possible reality, translated from a virtual experiential representation, must carry the traces of the process through which it was formed. This is not to say that the translation of form must be literal, as in Eisenman's Virtual House, but rather experiential; the spatial qualities of the architectural proposition must inspire in the viewer a common experience as in the digital representation that precedes it. Since this digital representation of experience need not conform to conditions of the real, the challenge for the architect is to capture in solid form the ephemeral qualities of this process. The result is an architecture generated from experience, realized and developed through the application of digital technology. 


\section{The Project}

This design project employs the digital tool in the design of a residential mixed use building sited in Ottawa, Ontario. The digital medium is used to develop the architecture through experience: animation is used in the project as a mode of representation that approximates the viewer's sense of duration. This facilitates the projection of self into the space of design throughout the design process. The final architectural result is translated from this digitally created, animated experience.

The process of design is broken into three successive stages, each of which is accompanied by a digital model and a series of animations. The first stage consists of the translation of the physical site into a digital model; this translation highlights specific elements and phenomena of the real site, and does not seek to simulate or recreate its actual condition. The emphasis is placed on the activities, movements, and textural qualities that surround the site and contribute to its character. In the second stage, this digital site model is used, along with its 
animated paths and objects, as the canvas for the design of an architectural experience. The elements of the digital site inspire the addition of form, texture, and lighting. These additions are developed in conjunction with further animation, which, through its composition, creates a representation of a transforming experience. The qualities of this experience are the conceptual basis for the translation into building that occurs in the third stage. This translation results in an architectural model that incorporates considerations of program and construction into the experiential concept. 


\section{Site}

The site is located at 125 Hickory Street in Ottawa. Bordering the site directly on the east side is the O-train line, to the north, the Ottawa Humane Society, and to the east, a large parking lot used by Ottawa Civic Hospital staff. Other surrounding features of note are Dow's Lake, which is located approximately one half kilometer southeast of the site, the Queensway, which runs east/west one and a half blocks to the north, and Preston street, one

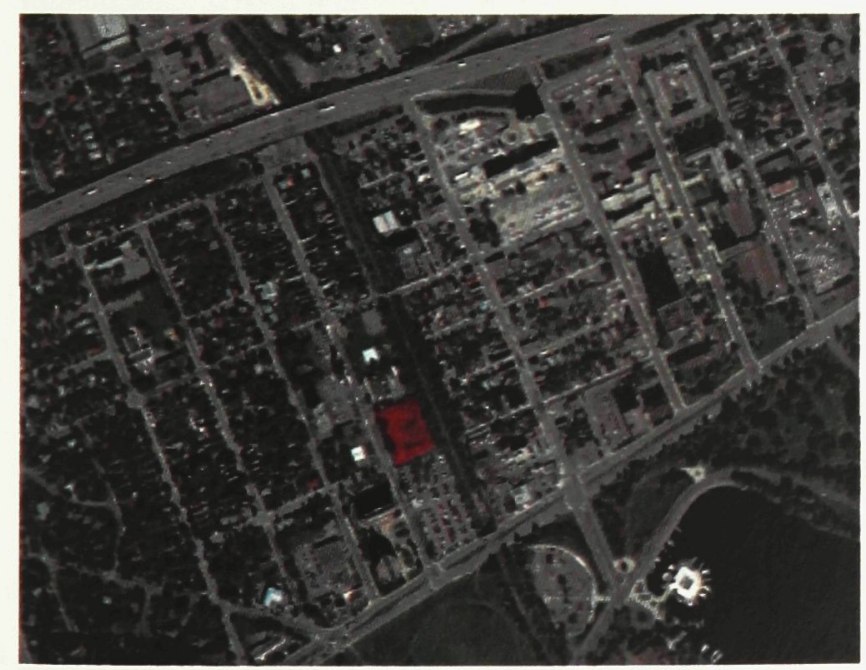

Fig. 15125 Hickory Street, Ottawa, Ontario, Canada. From: Google Earth, Sept 10, 2005. block east across the $\mathrm{O}$-train track, which runs through the heart of Ottawa's Italian district. The surrounding area is also characterized by a significant amount of open, green space, especially on the south side of Carling avenue, but including a small yet busy park at the corner of Beech street and Champagne avenue. 


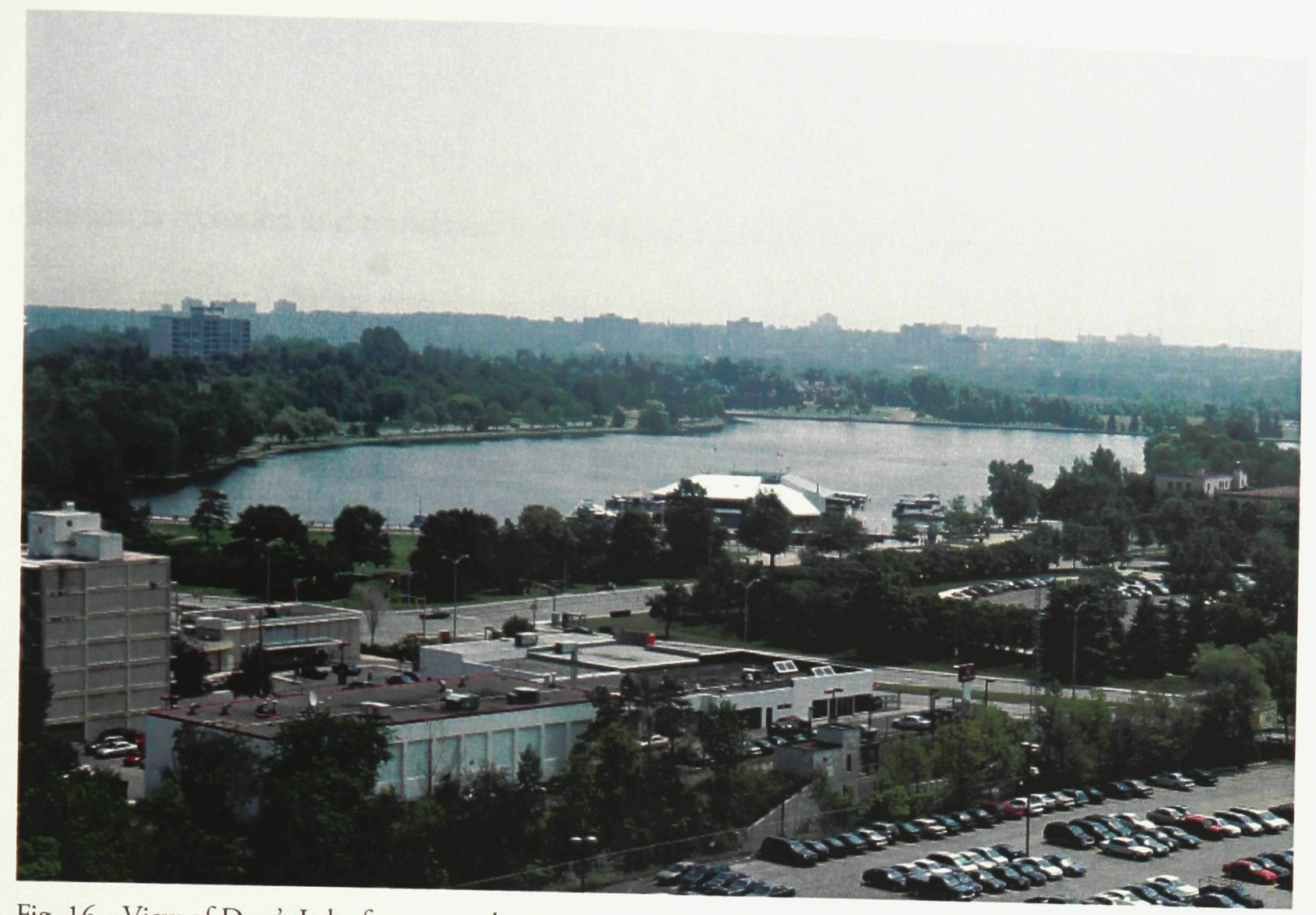

Fig. 16 View of Dow's Lake from near the site.

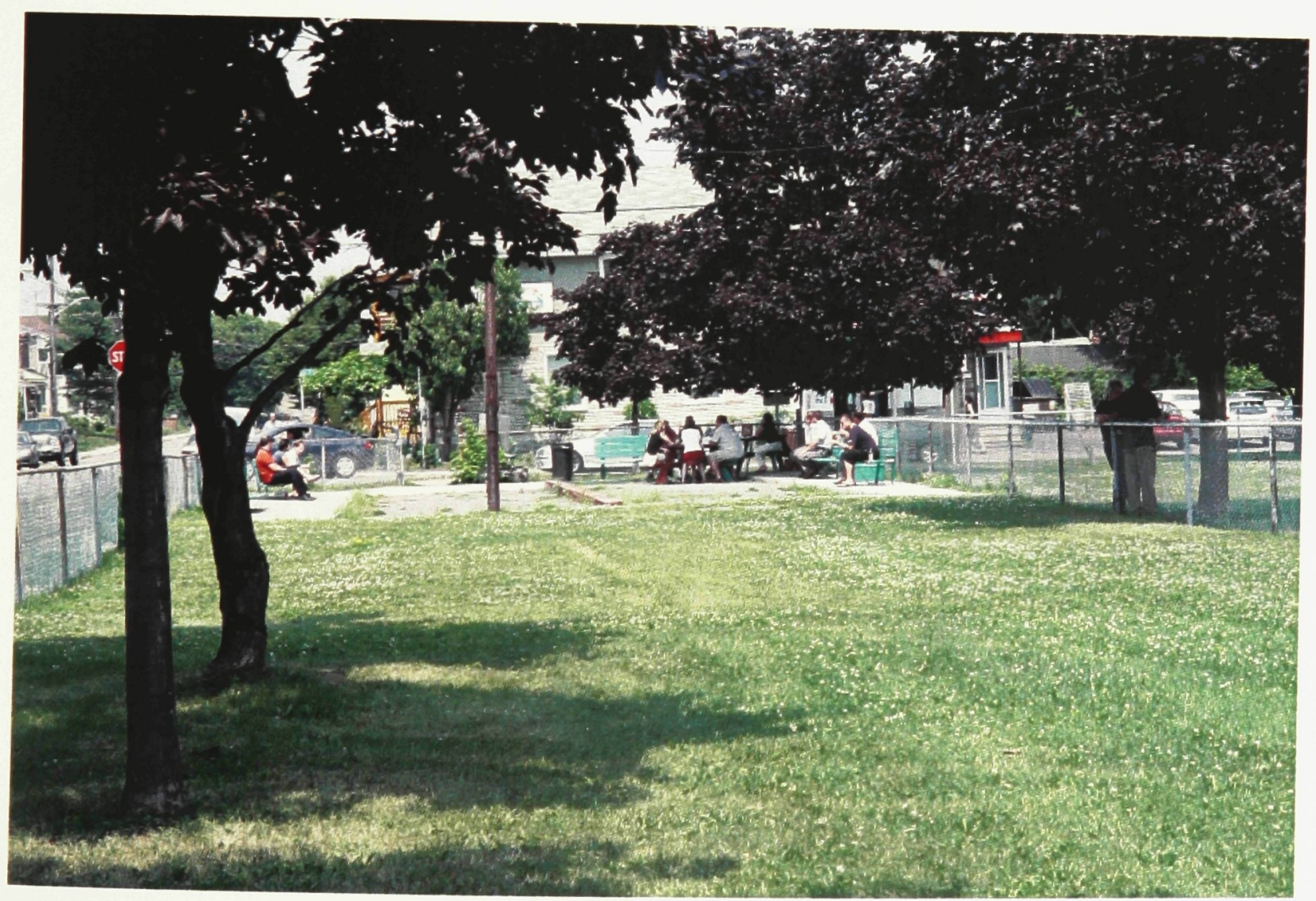

Fig. 17 Park at the corner of Champagne avenue and Beech street. 
The site is recorded and translated into a digital model with an emphasis placed on the mobile, creating a digital environment that conveys the qualities of the site that give it "life". For example, the two major traffic arteries in the area, Carling avenue and the Queensway, are mapped with animated textures that define their high speed motion, and establish them as threshold conditions in the urban context. The O-train assumes the form of a streak of color, which flashes momentarily, and highlights the void space that exists in its absence. The trees that define much of the surrounding green space form a semi-transparent canopy of texture. These types of ephemeral representation are combined with stable elements that orient the viewer to the site. The surrounding buildings are represented as translucent masses, and the contours, which are in fact already an abstraction of ground, suggest the landscape.

A series of camera paths is introduced during the creation of this model, which focus on revealing these various site conditions. The animation of the camera along the path creates a moving viewpoint that presents the space of the model in perspective and in time. The combination of the moving viewpoint and the animated site phenomena forms the basis for the creation of a virtual experience of the site model. Perceived in duration, elements of the animations draw on the viewer's memory of past experiences in order to allow for the mental synthesis of a current virtual experience. The subjective reading of the site model through the animations establishes the important events and moments that the second model is founded upon. 


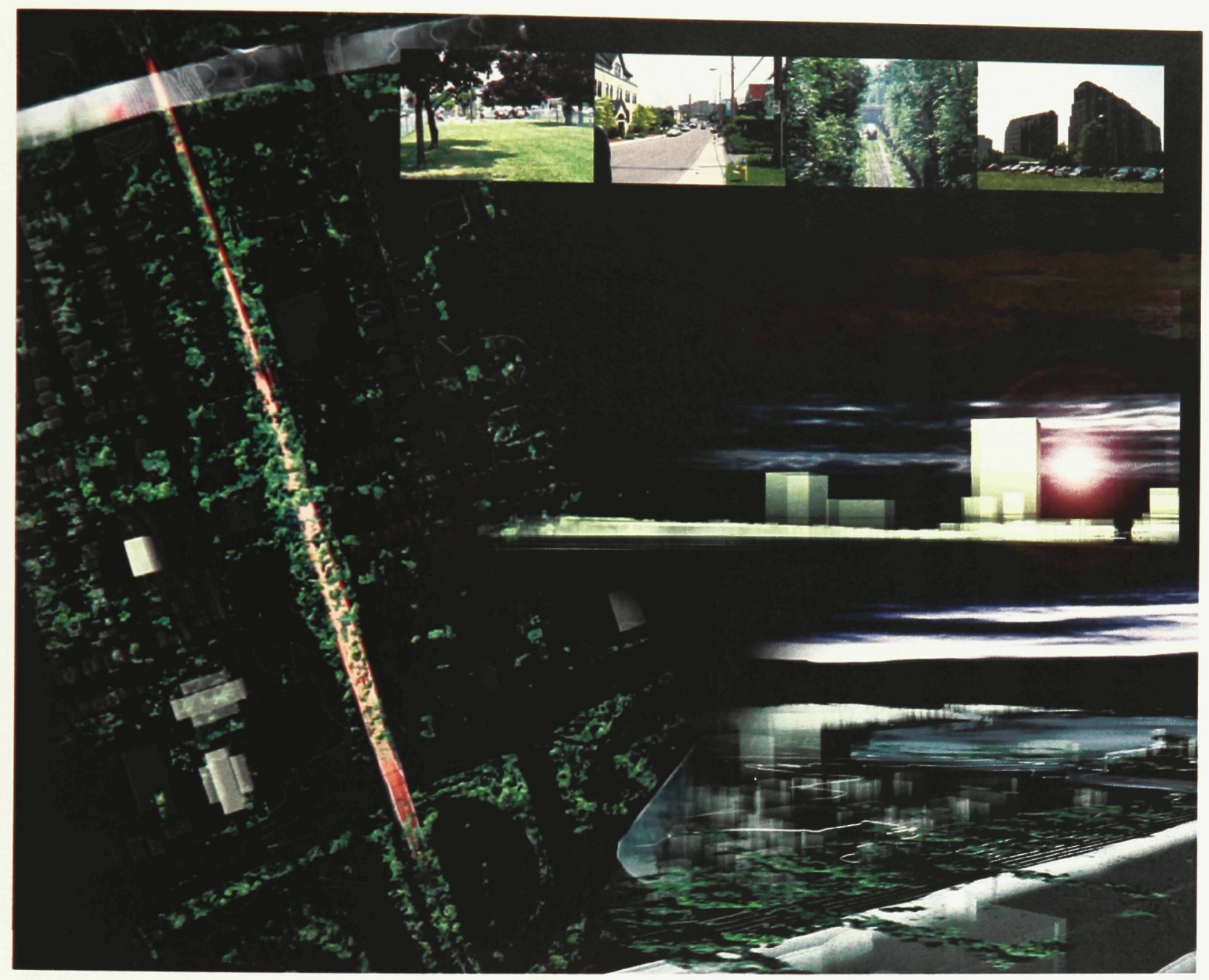

Fig. 18 Digital site model. 


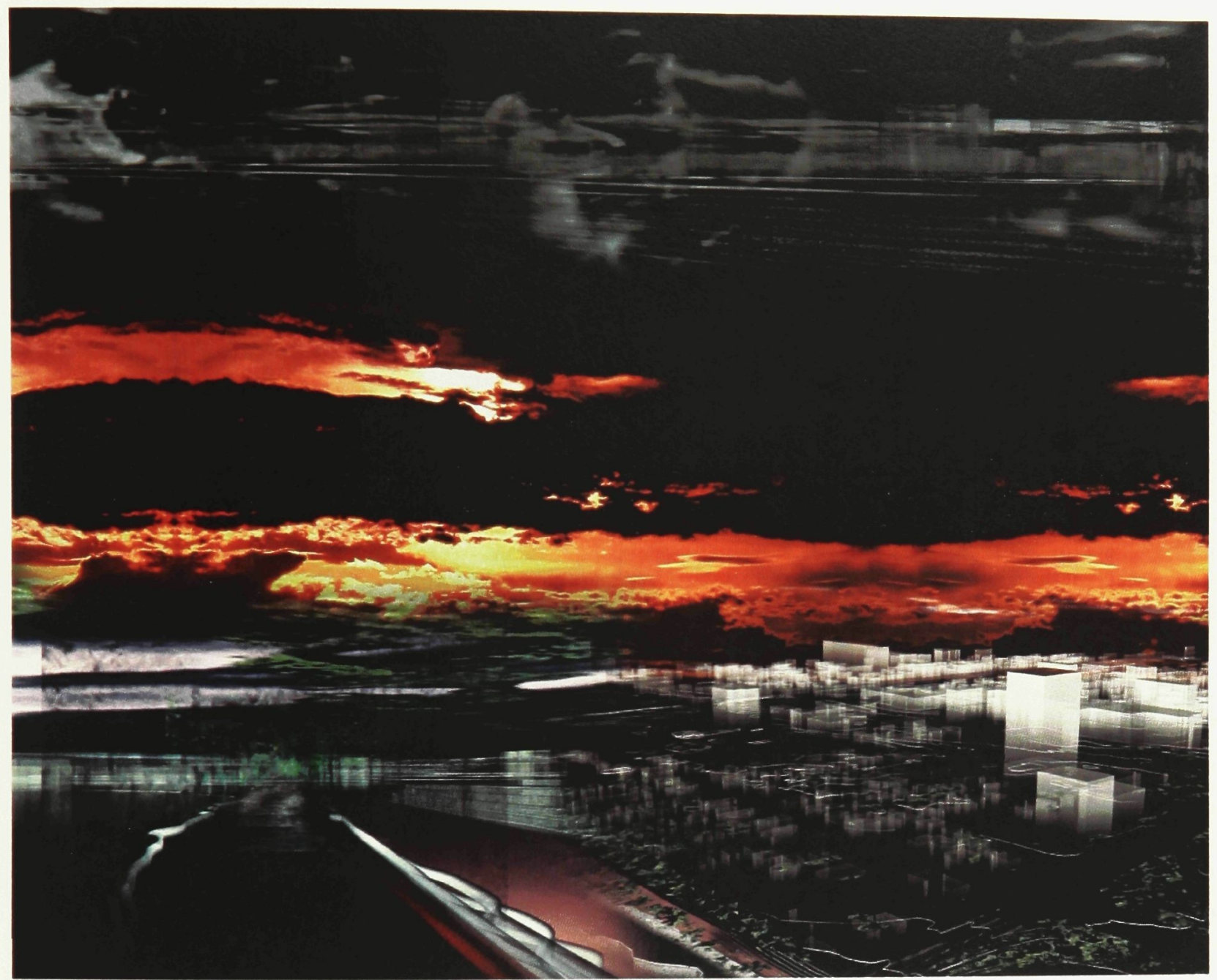

Fig. 19 Digital site model. 


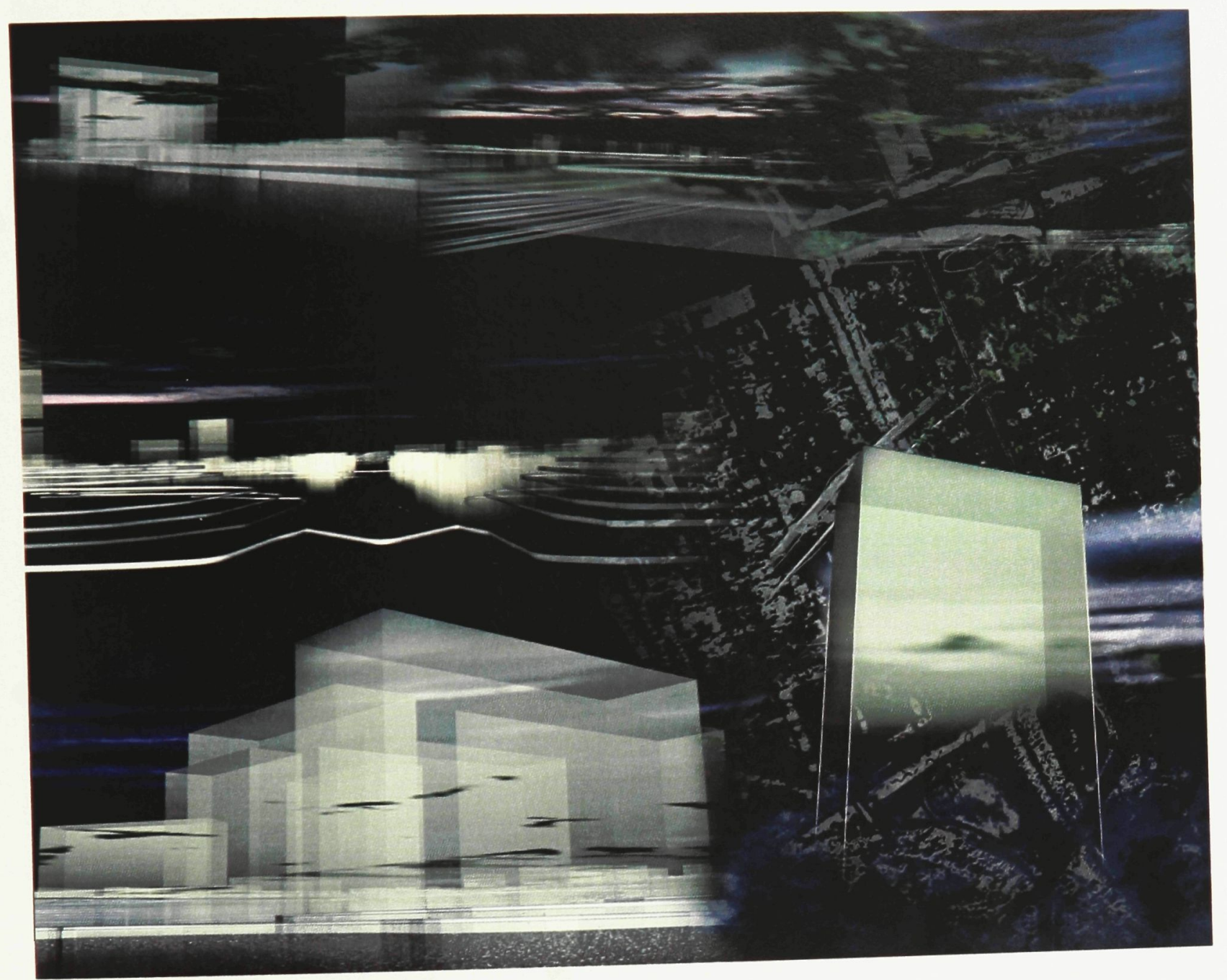

Fig. 20 Digital site model. 


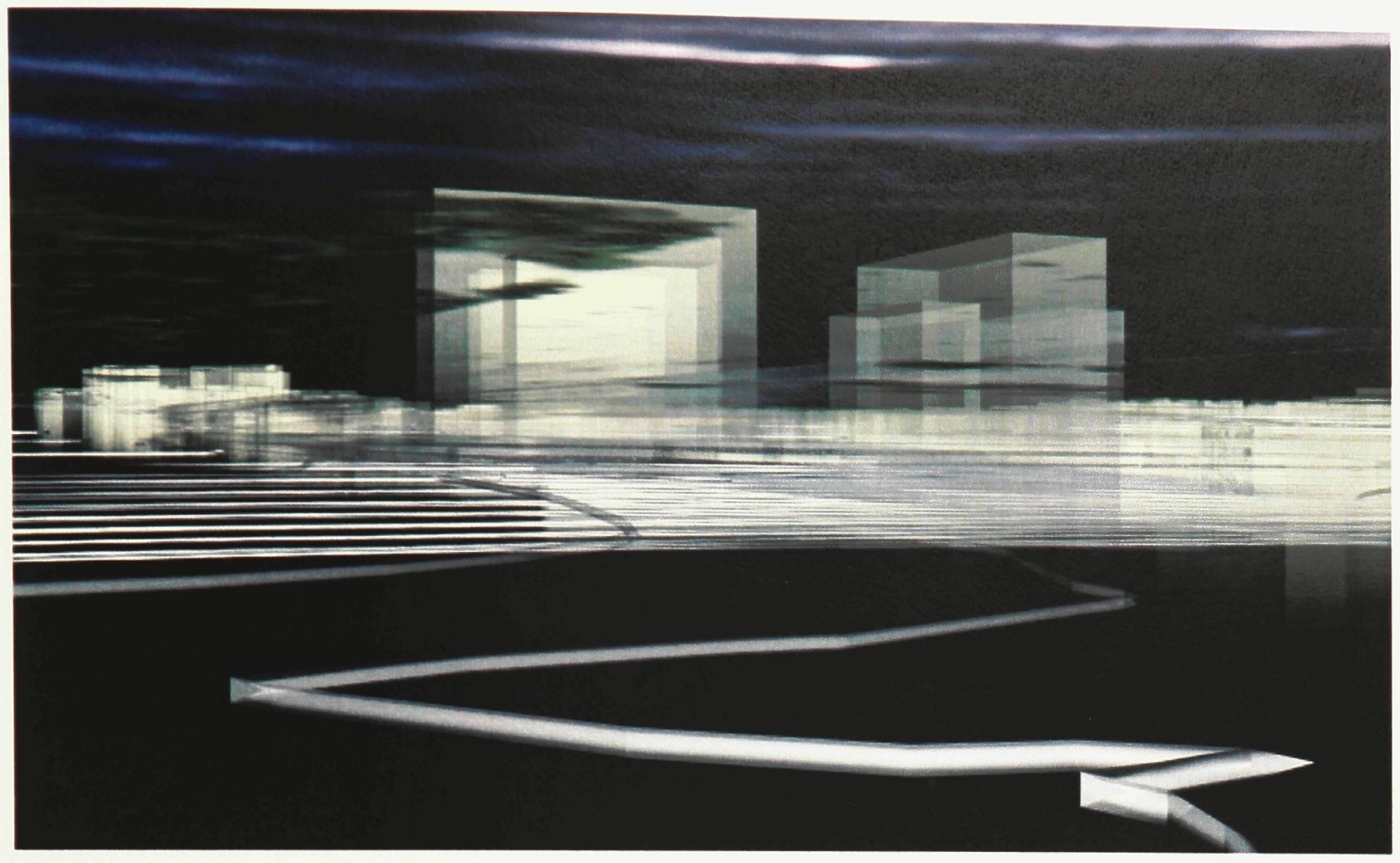

Fig. 21 Approaching Carling avenue. Digital site model.

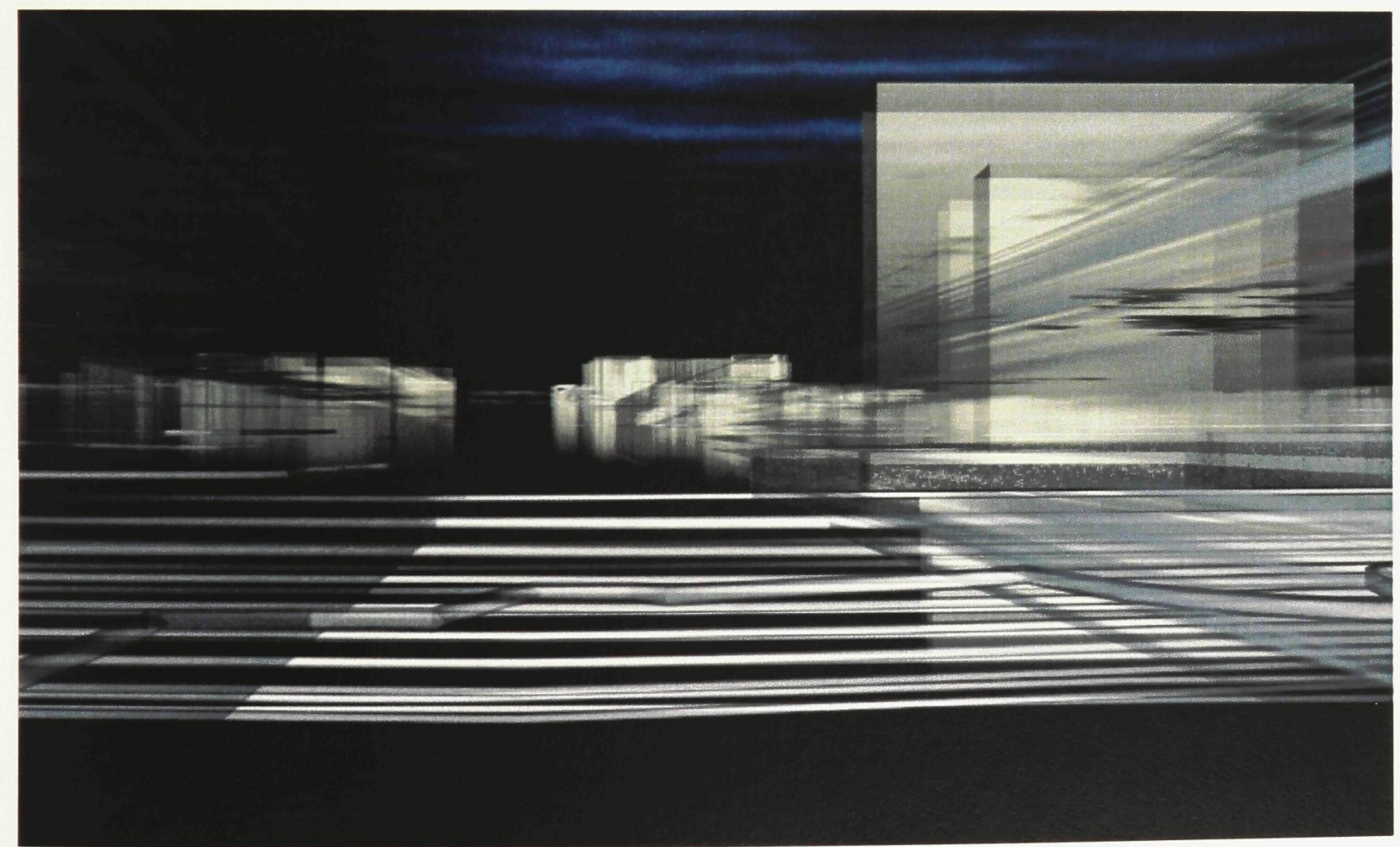

Fig. 22 Carling avenue. Digital site model. 


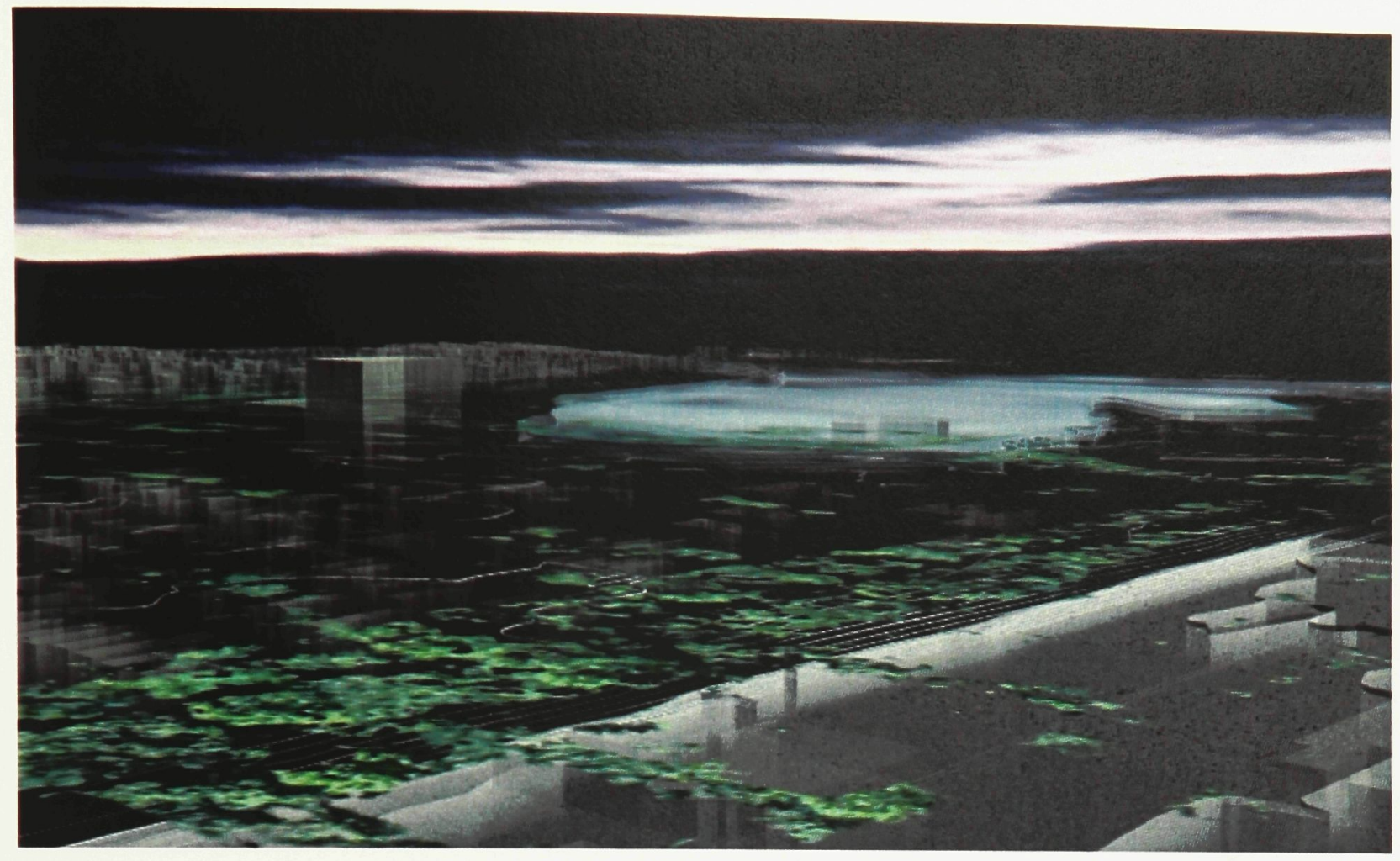

Fig. 23 View towards Dow's Lake. Digital site model.

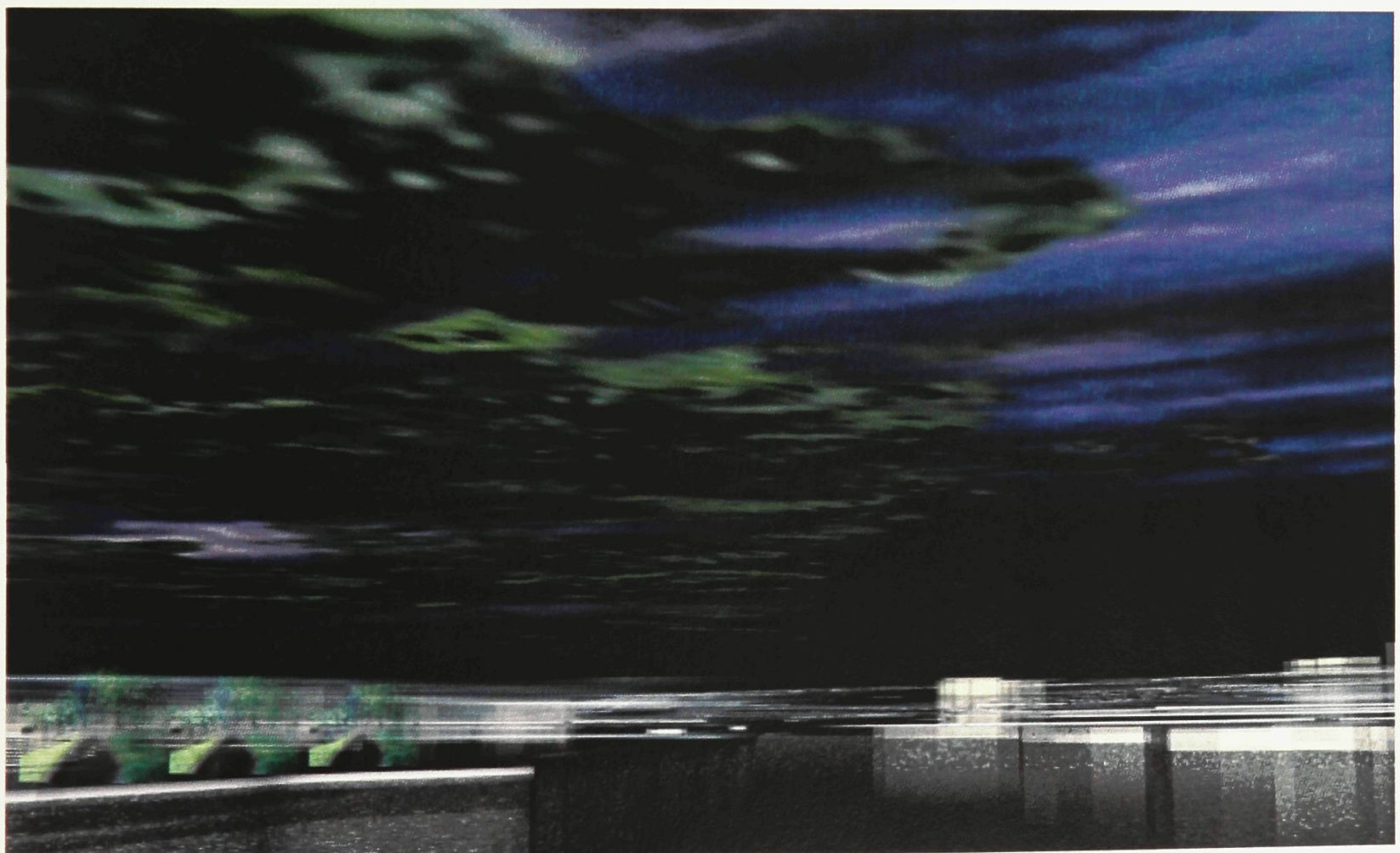

Fig. 24 Looking towards Carling avenue. Digital site model. 


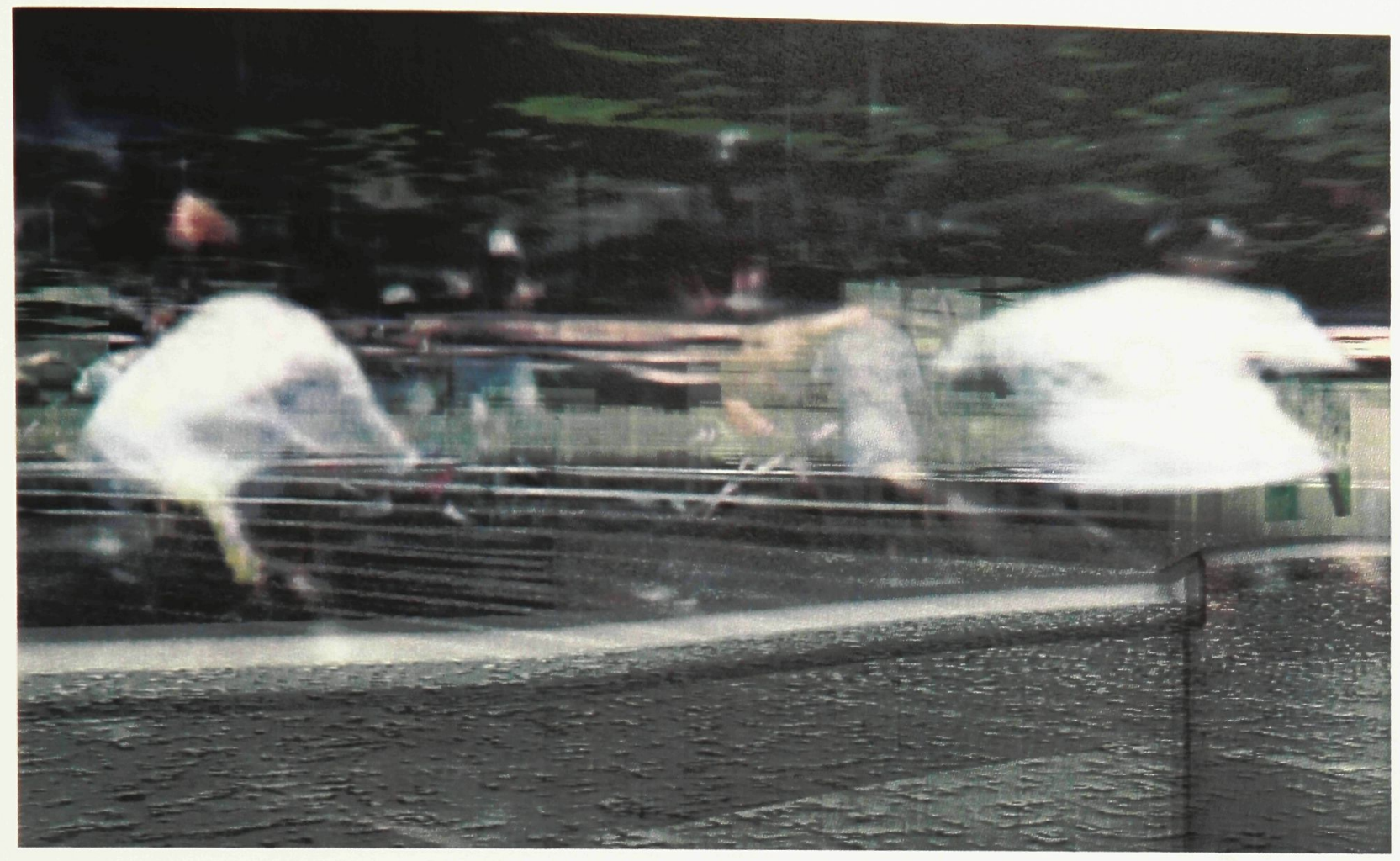

Fig. 25 The park at Champagne avenue and Beech street. Digital site model.

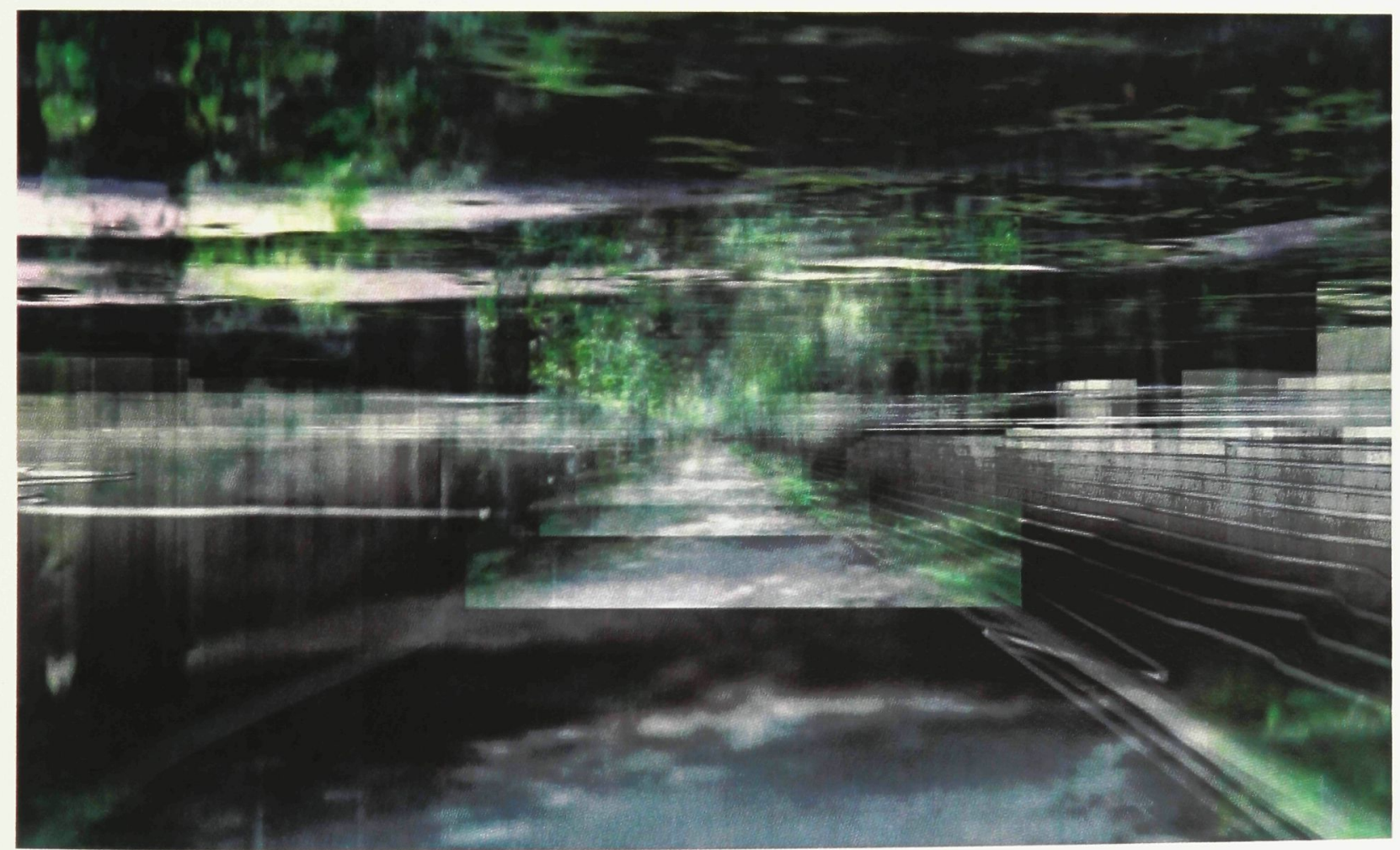

Fig. 26 Pathway on the east side of the O-Train track. Digital site model. 


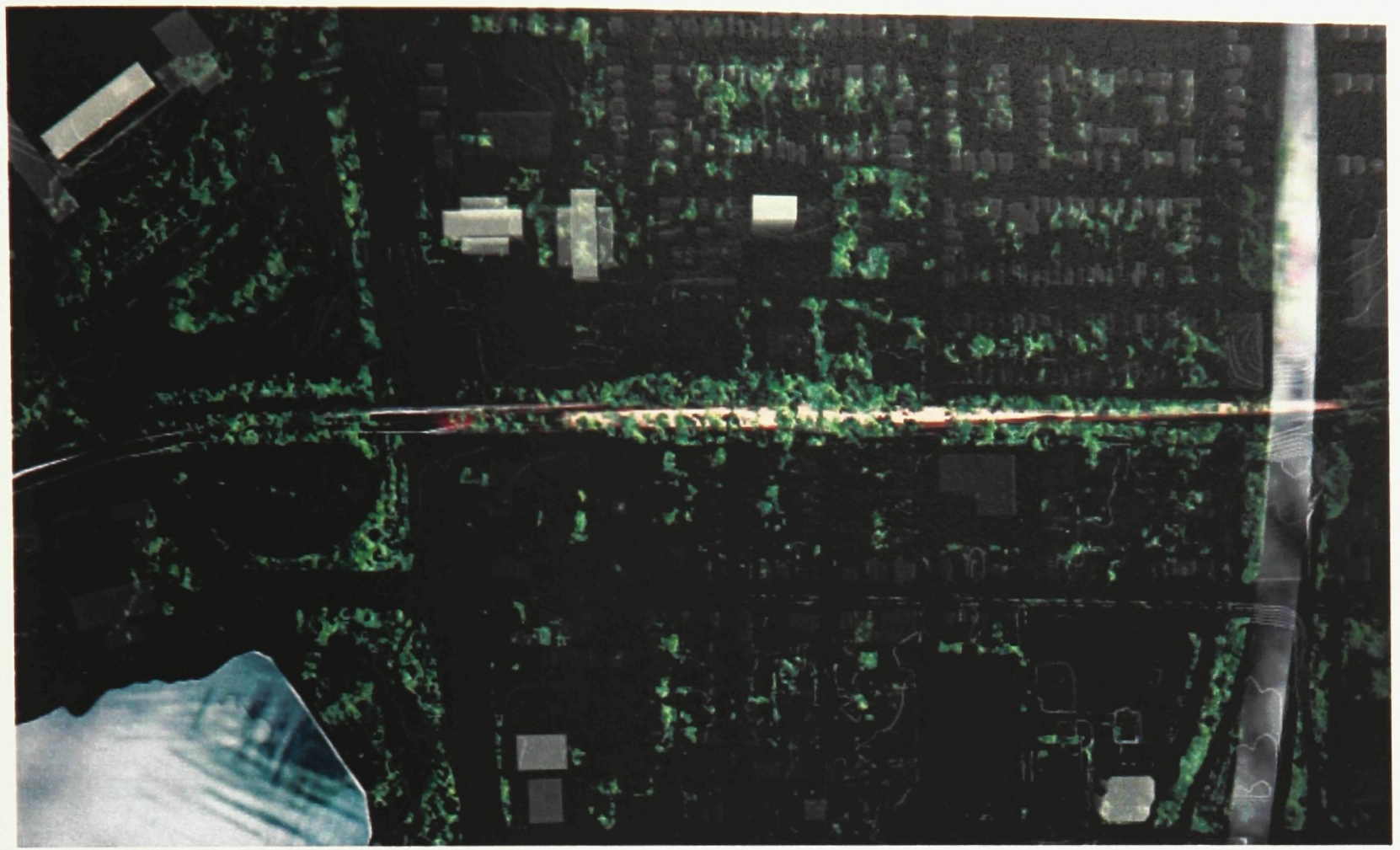

Fig. 27 Plan view of site. Digital site model.

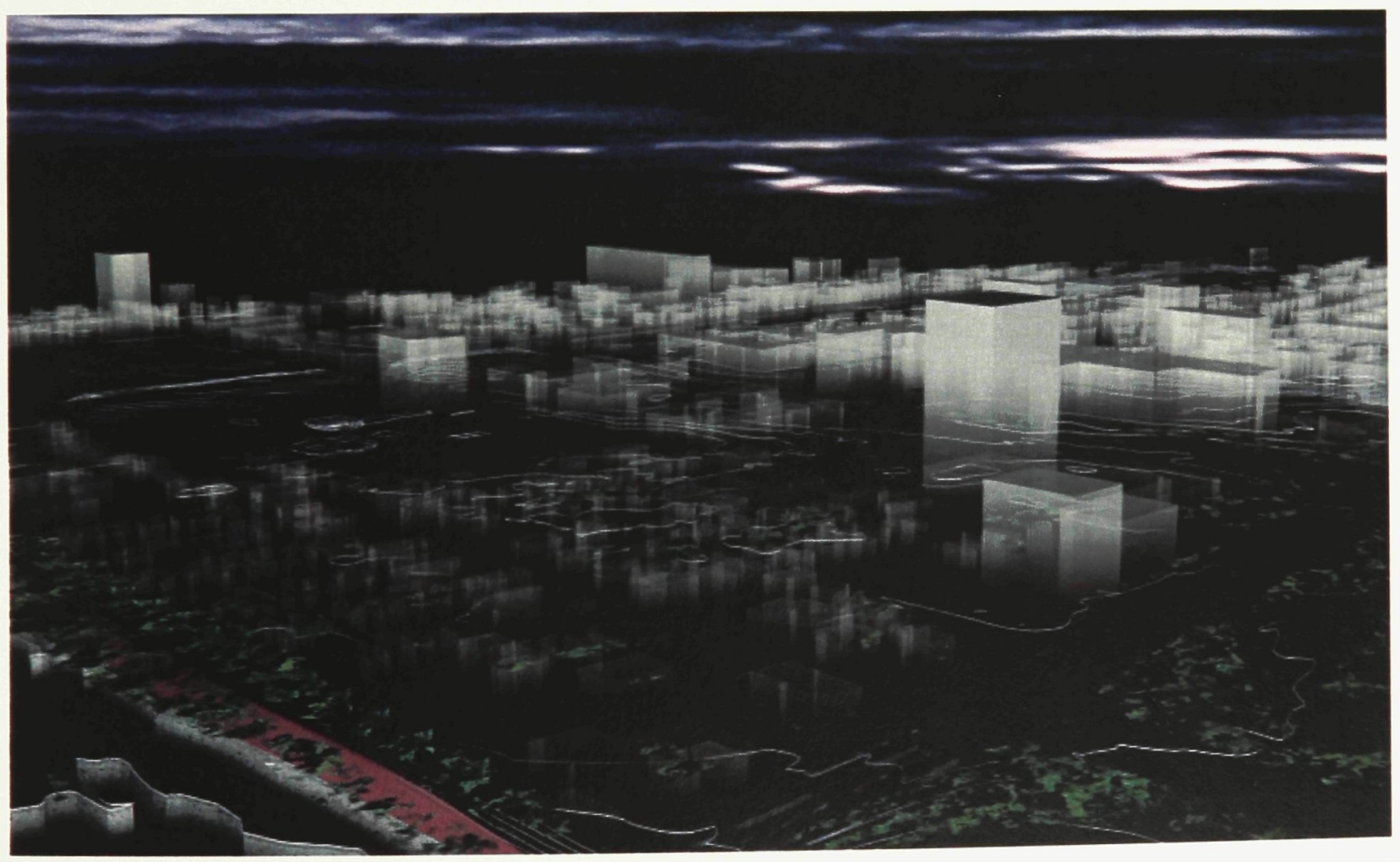

Fig. 28 View towards the city to the northeast. Digital site model. 


\section{Experience}

The second phase of the project consists of the construction of a digital model that responds to the animated site condition established in the first model. While architectural qualities of light, space and materiality play a key role in the development of this second model, none of the restrictions of the physical site are placed on this process. Rather, the digital site is considered to be the only generator, or "ground", on which a representation of experience is developed. The goal of this intervention is the exploration of a response to the site model's phenomena, with specific emphasis placed on framing, interruption and accentuation. The site model is explored and altered through the creation of new animations, which establish a virtual experience for the viewer.

Taking the site model as the inspiration, I began by watching the animations of the site. The first move in the construction of the second model was the selection of a camera path from the first model. A path was chosen that surveyed the surrounding area from the center of 
the site. Forms were placed around the camera path in order to frame out the surrounding buildings and highlight the canopy of texture and the sky beyond. The forms were built looking through the camera as it moved. This created an array of distorted forms generated in reaction to a desired experience of the site. A second camera path was chosen, which now surveyed the site model as well as the interventions added to it. The subsequent constructions began to focus on the relationship of each successive layer to its predecessor as well as to the original site model.

During the construction of this second experiential model, I worked exclusively through perspective in the software interface. By restricting my interaction with the software in this way, I was able to perceive the model at all times as an environment, rather than as a distinct object. Because the perspective viewpoint is sympathetic to the function of my own eye, this method of working allowed me to construct the environment around myself, encouraging an experience of space and inhabitation even prior to attaining the rendered animation.

Because the conventions of orthographic projection were ignored, the resulting model is not intended to be viewed as an object from an exterior vantage point. Rather, it is presented as it was conceived: from within. The spatial and experiential qualities evoked by the images and animations generated through this model then served as the conceptual basis for the architectural resolution that the third step requires. In essence, this second model has achieved the status of a conceptual sketch which the viewer can inhabit through the digital medium. 


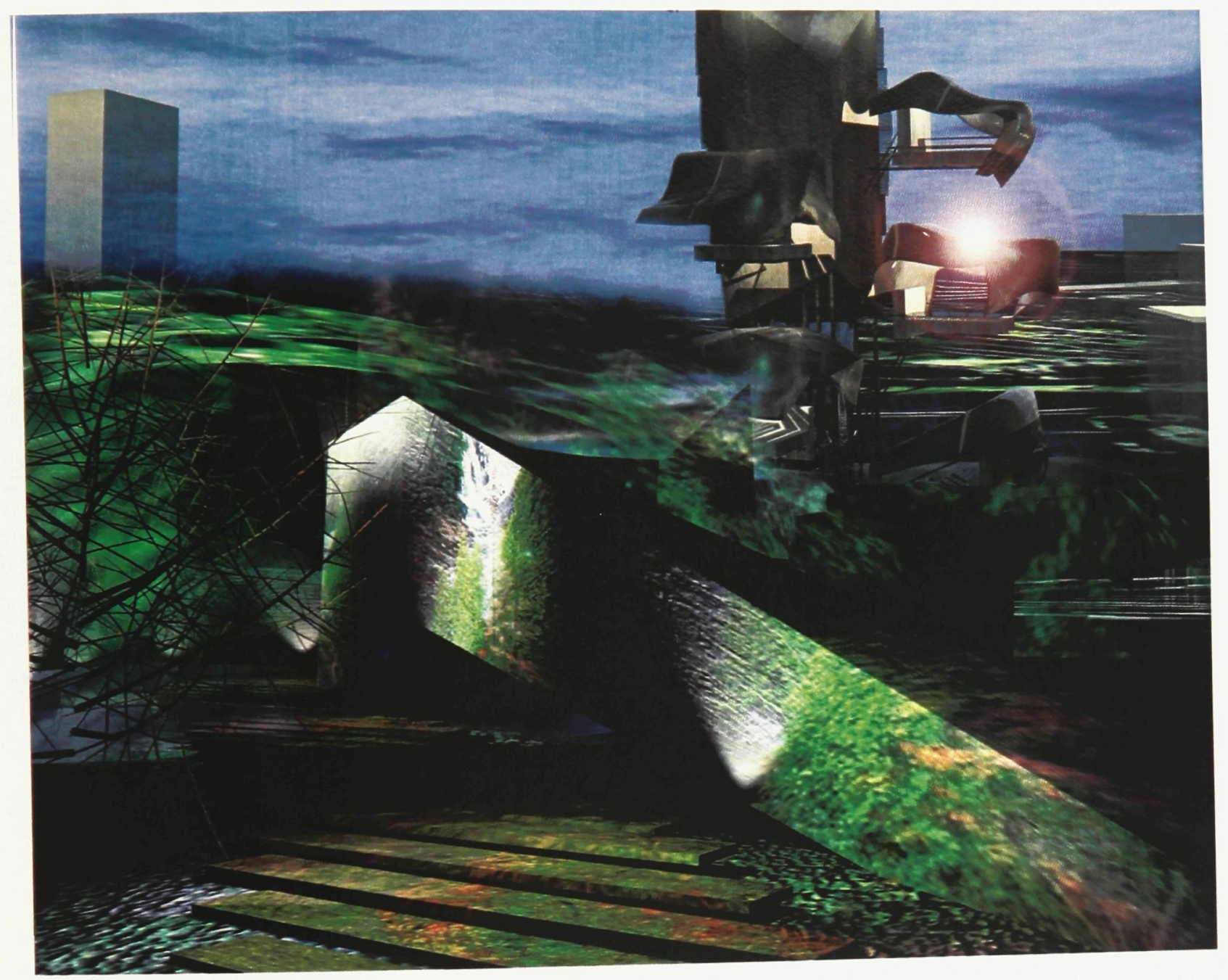

Fig. 29 Digital experiential model. 


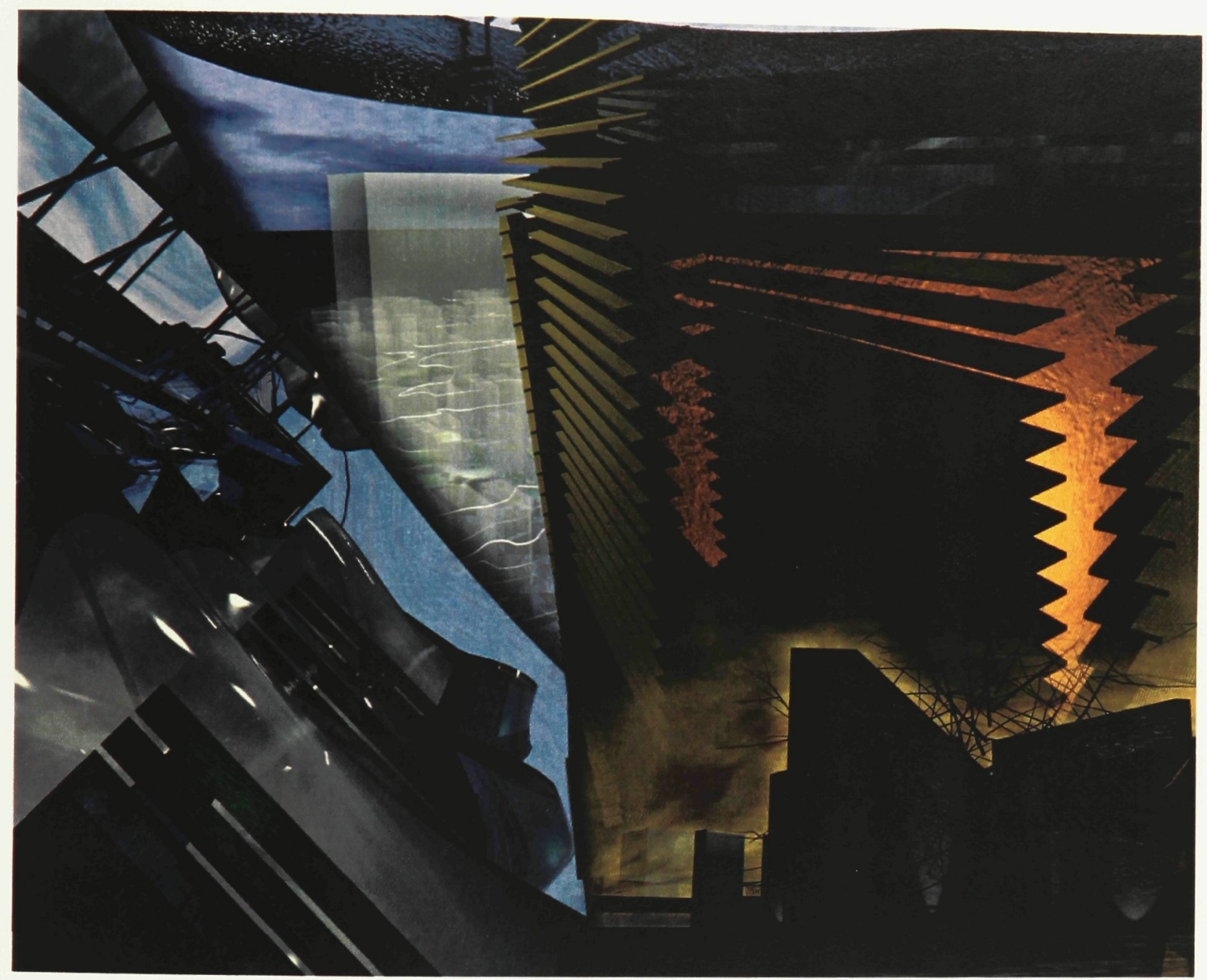

Fig. 30 Digital experiential model. 


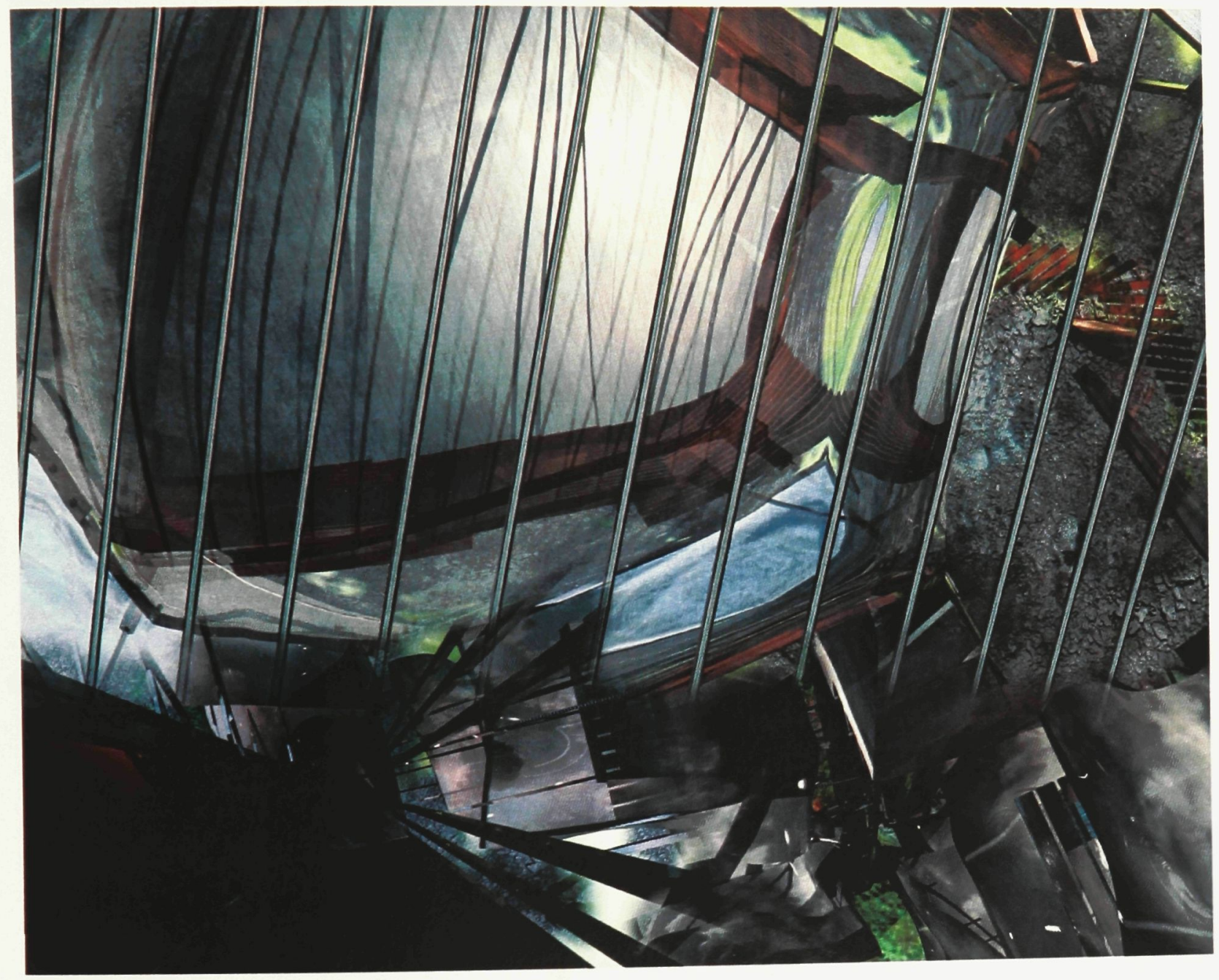

Fig. 31 Digital experiential model. 


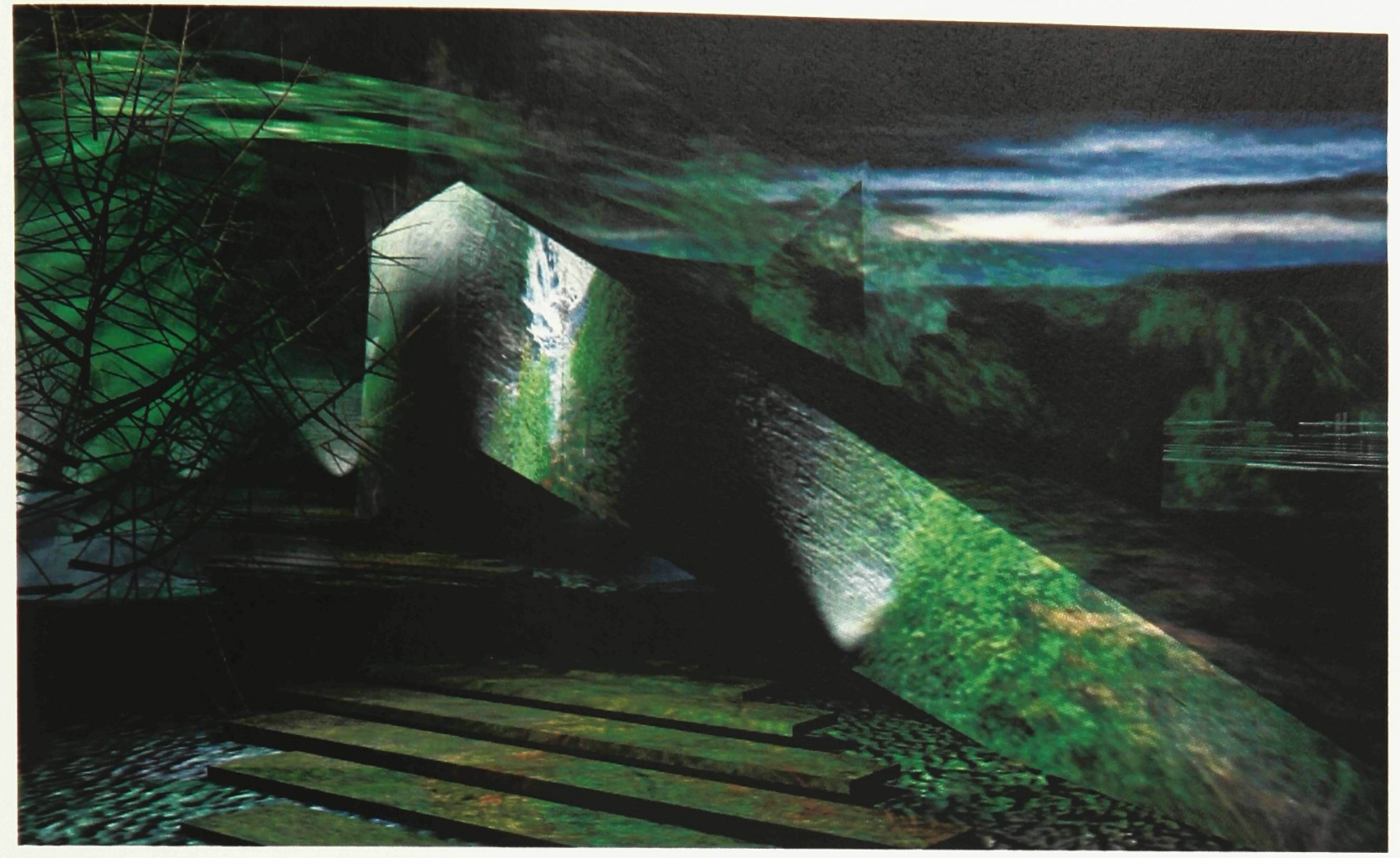

Fig. 32 Digital experiential model.

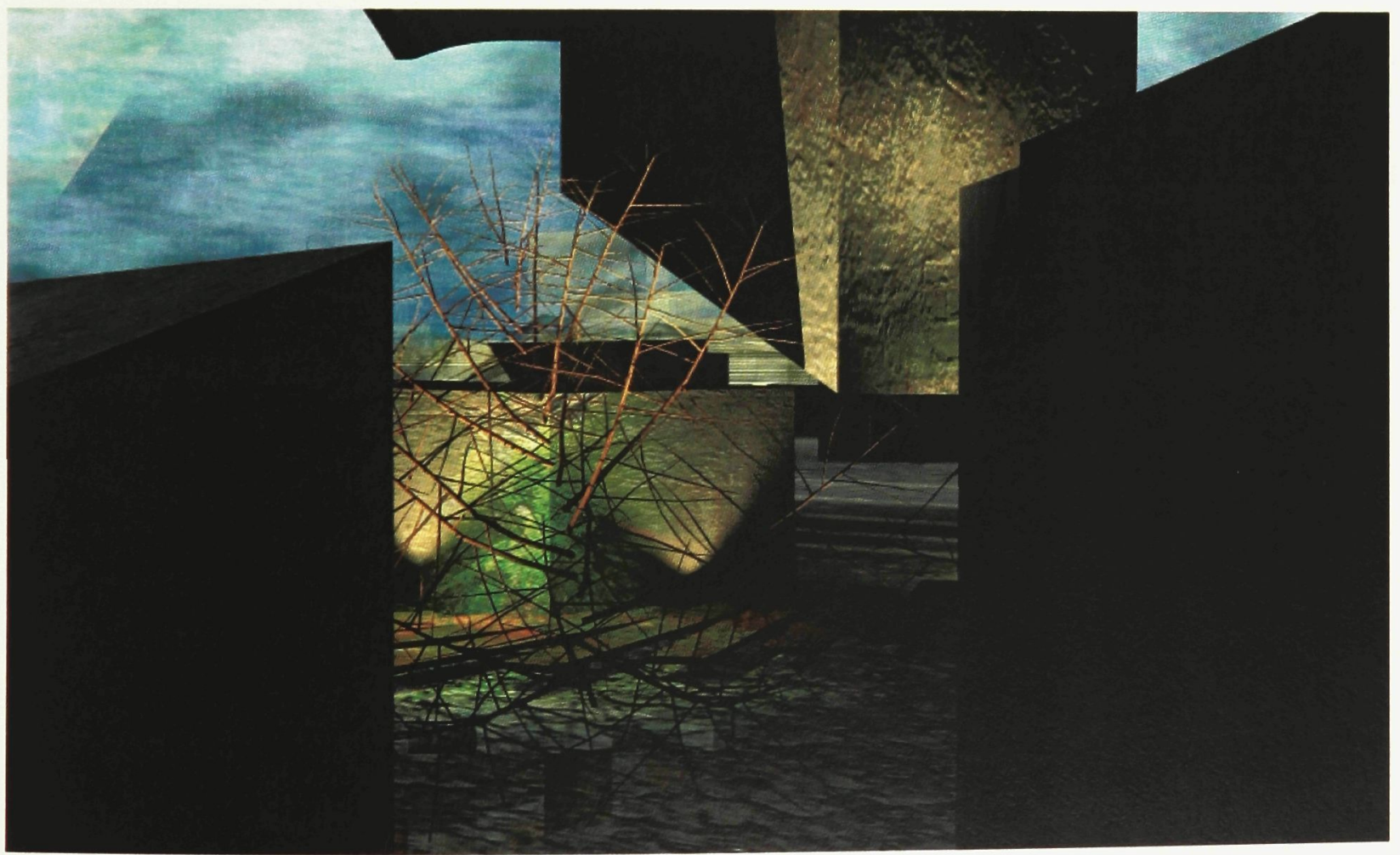

Fig. 33 Digital experiential model. 


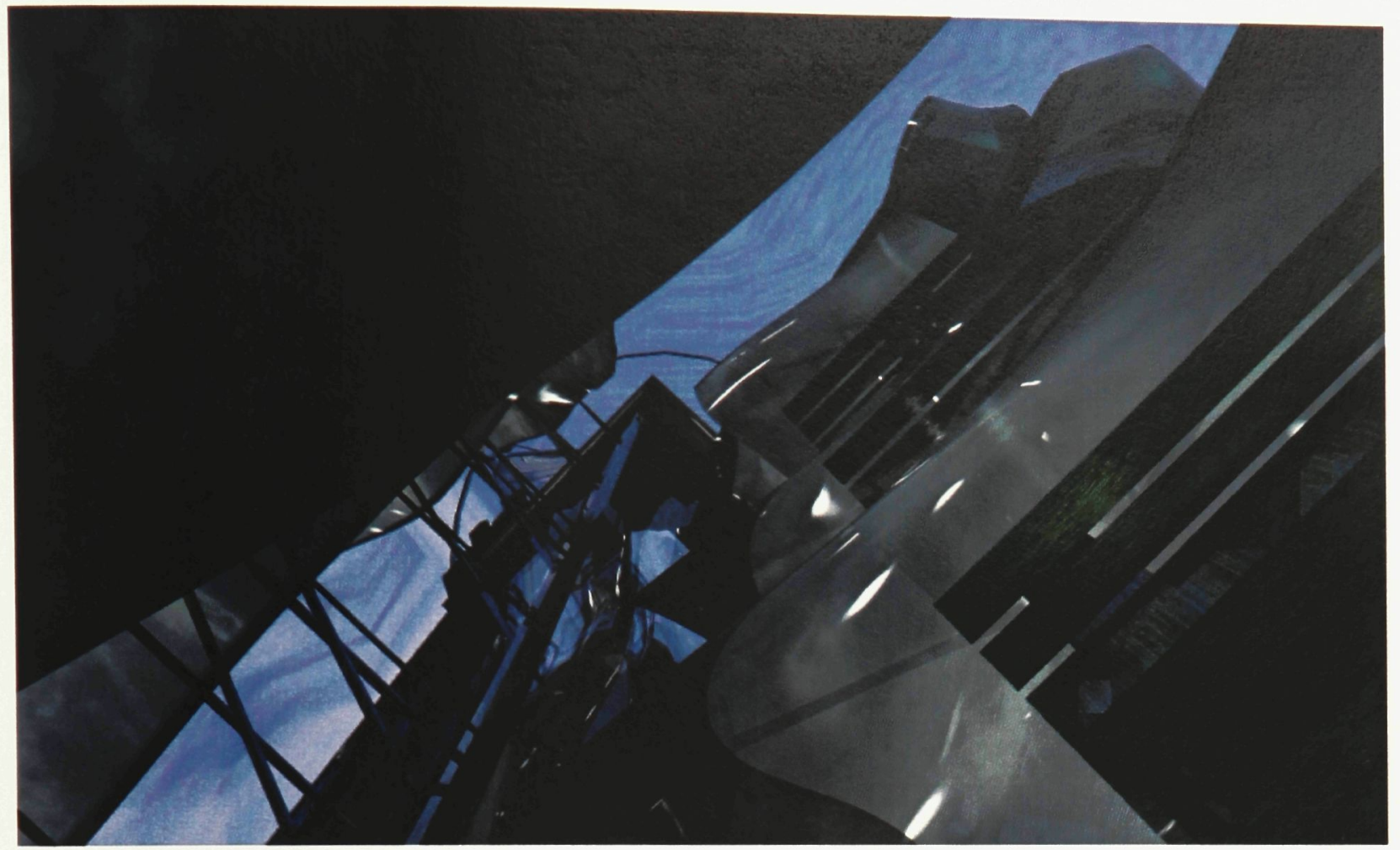

Fig. 34 Digital experiential model.

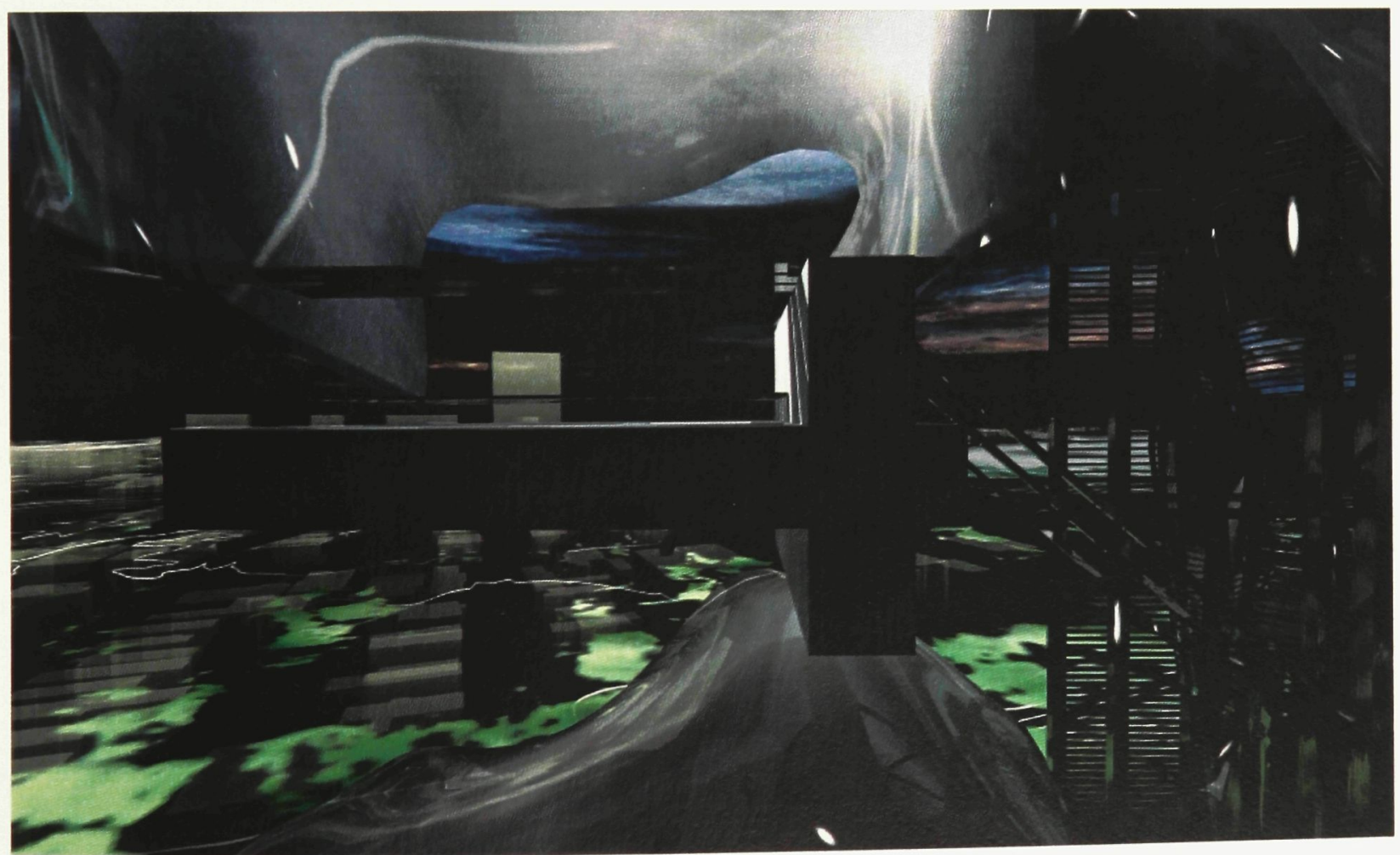

Fig. 35 Digital experiential model. 


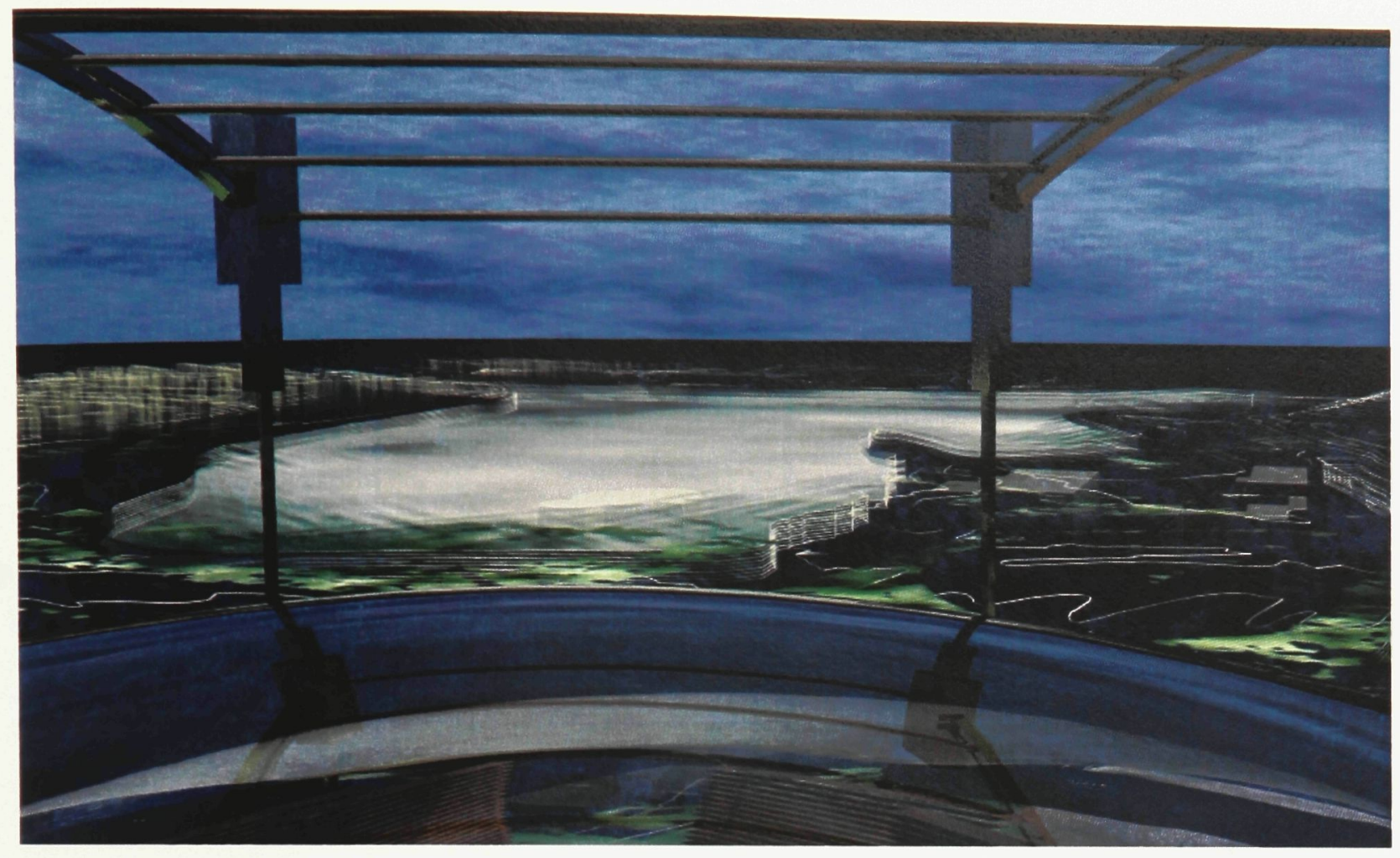

Fig. 36 Digital experiential model.

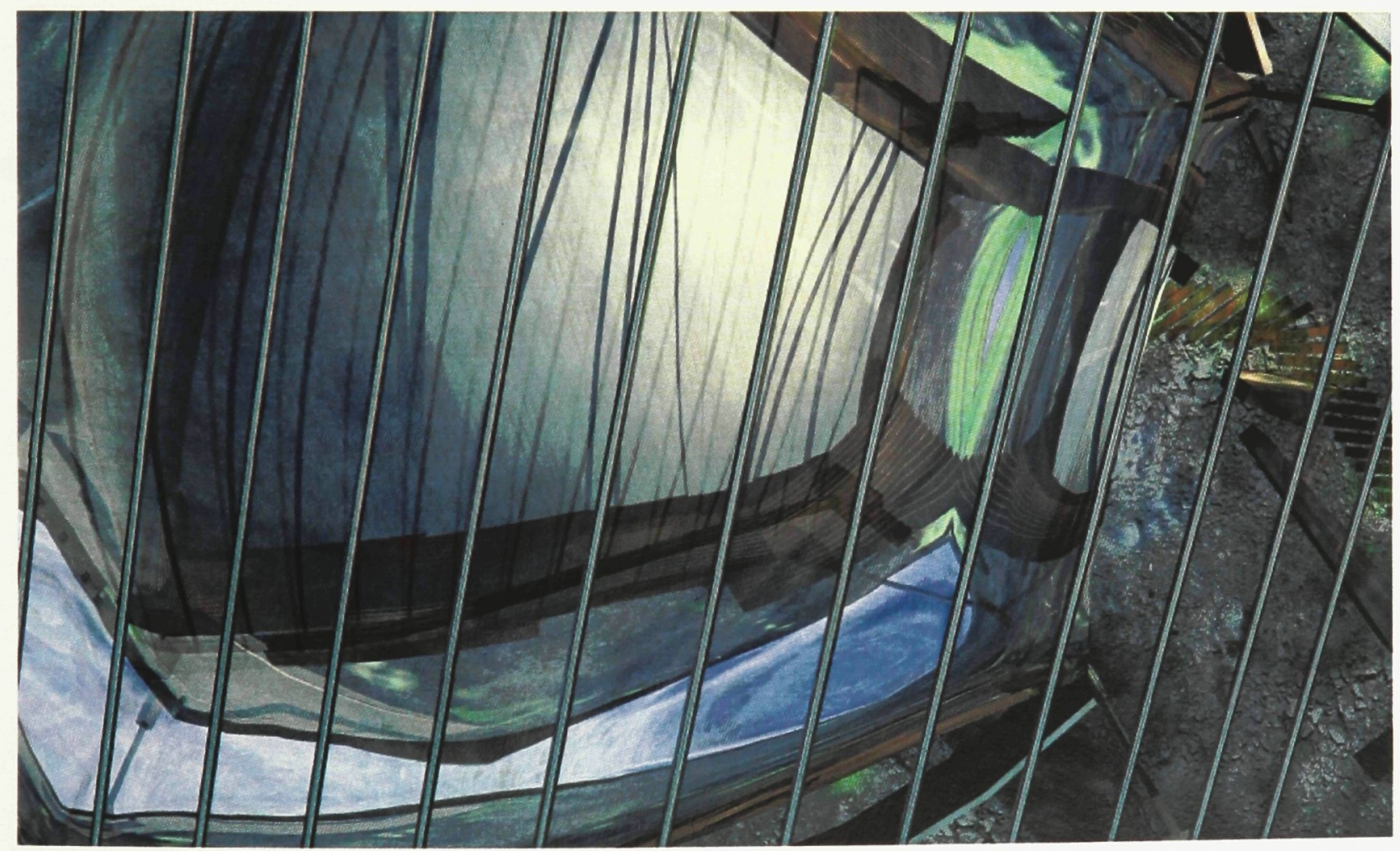

Fig. 37 Digital experiential model. 


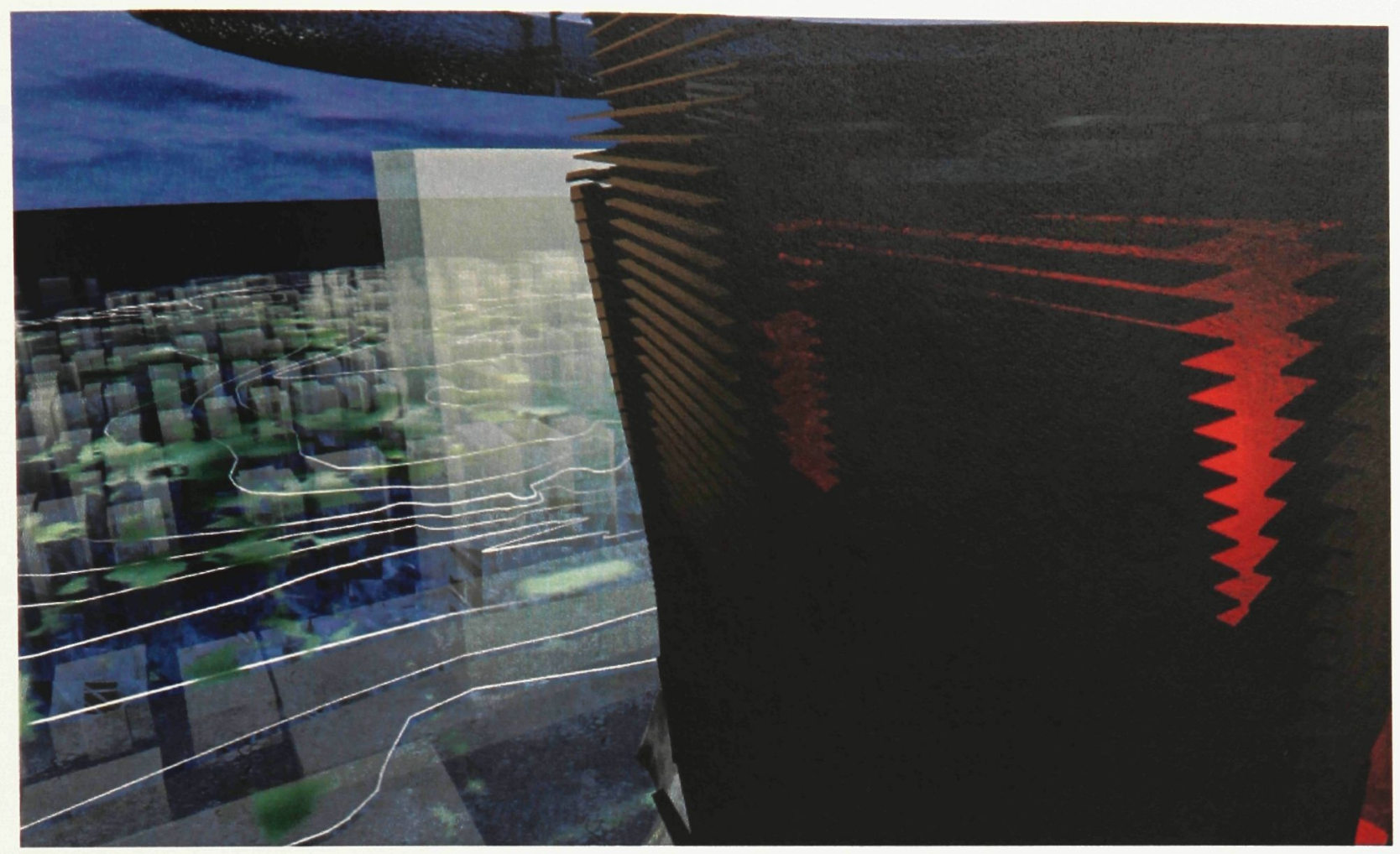

Fig. 38 Digital experiential model.

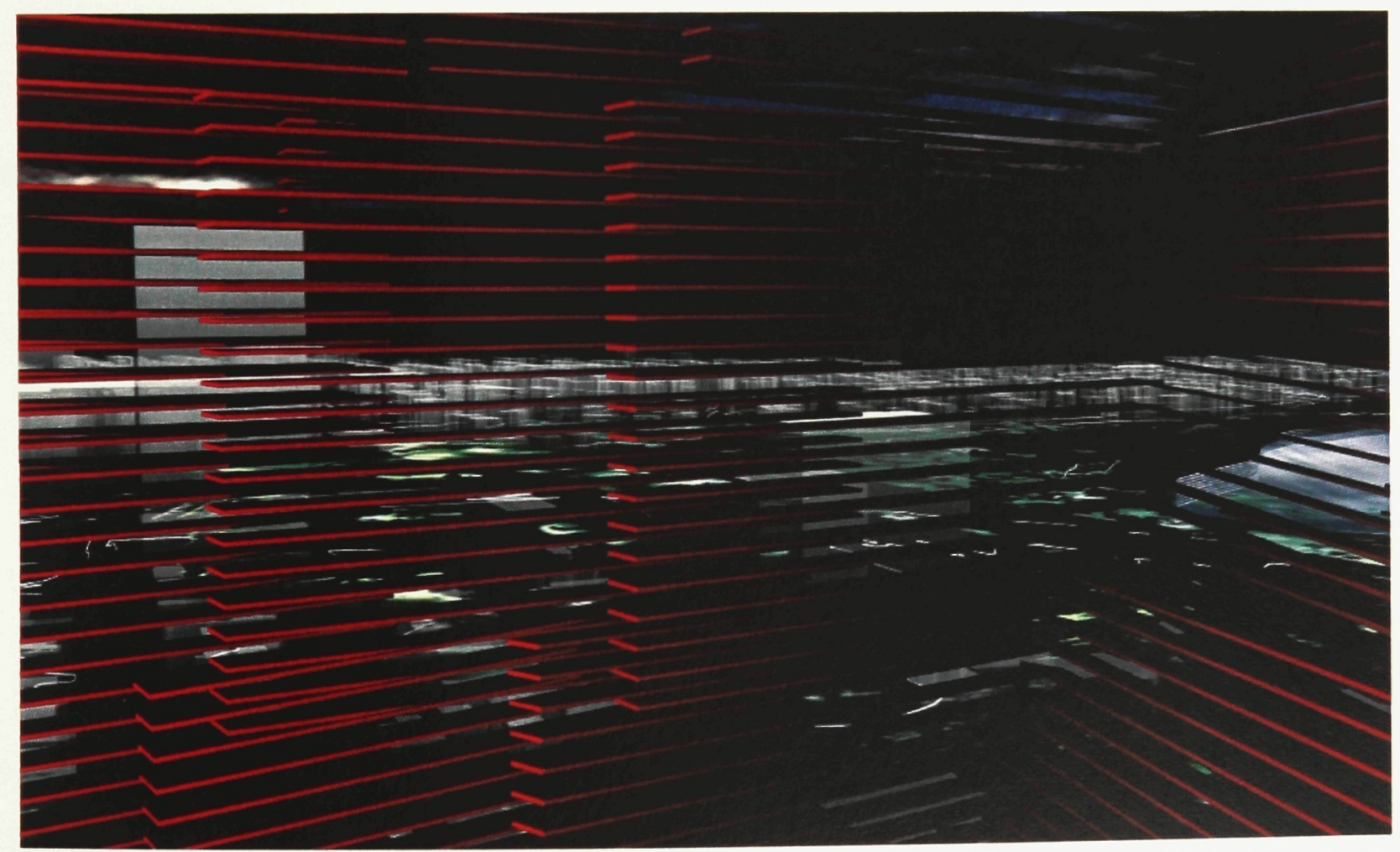

Fig. 39 Digital experiential model. 


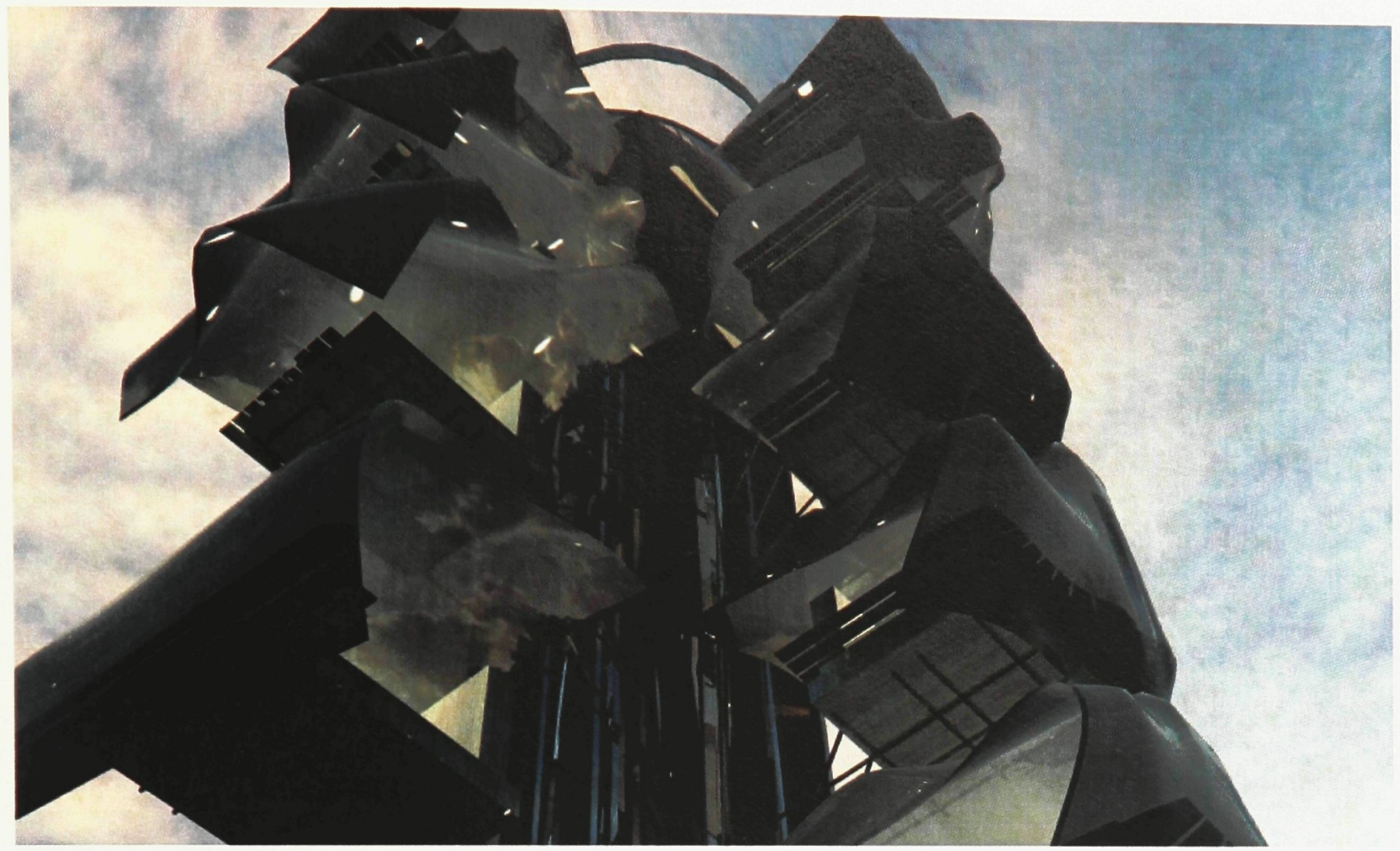

Fig. 40 Digital experiential model.

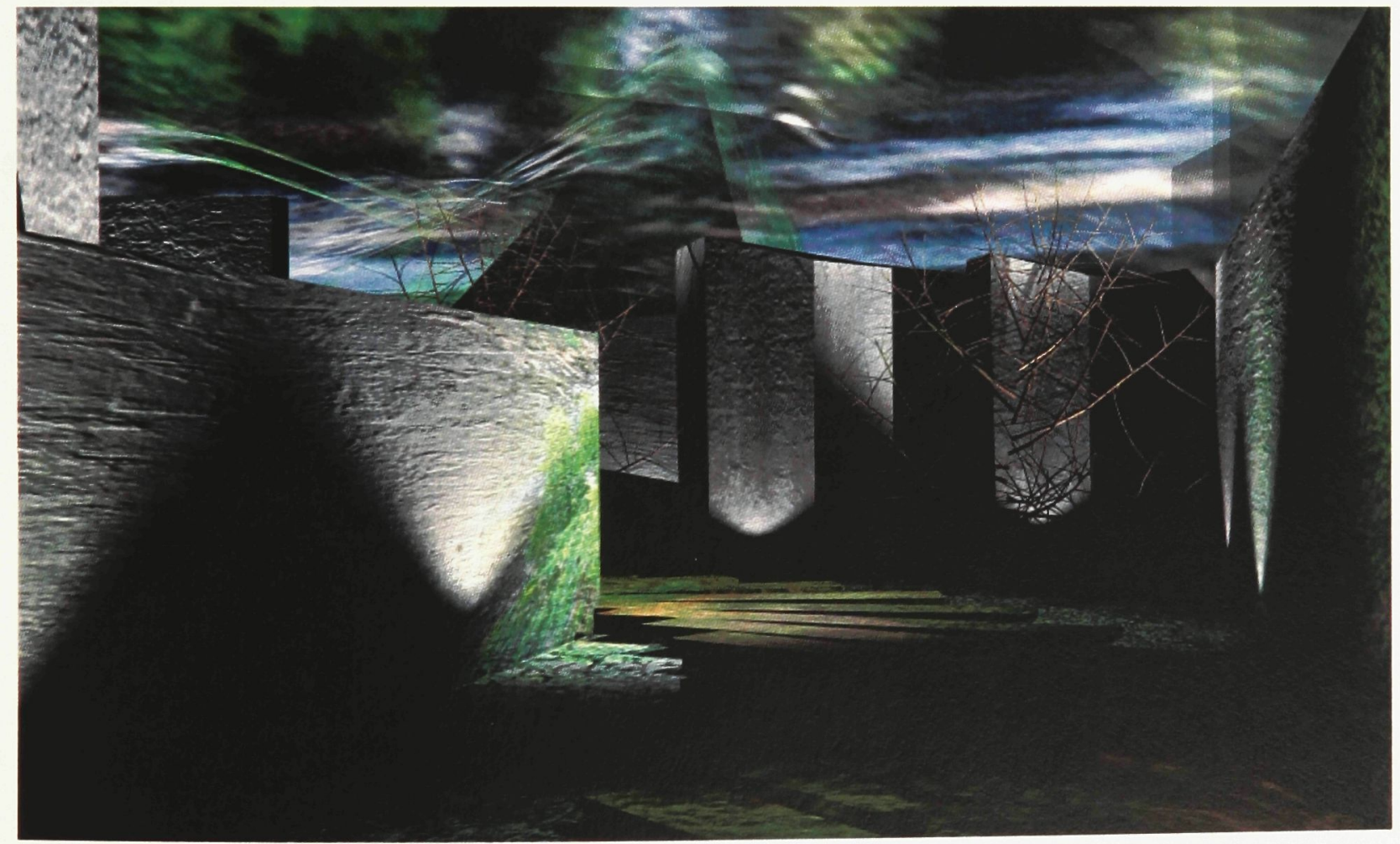

Fig. 41 Digital experiential model. 


\section{Architecture}

The final stage of the project is the translation of the digital conceptual sketch into an architectural project. Ultimately, what is sought is a design that balances the conceptual imaginings of the experiential model with the practical requirements of program, structure and constructability. The translation that takes place through the digital medium is not the structural resolution of complex geometries and anamorphic forms; rather, the digital tool is used to investigate the qualitative spatial properties of the model as it is designed.

As such, the goal is not to design a building that necessarily "looks like" it was designed on a computer. The digital tool is used here as a tool for exploring the interaction of light and material, of texture and form, and of spatial inhabitation, rather than as a tool for the structural resolution of complex geometry. Throughout the design of the architectural project, walkthrough viewpoints were used to explore the spaces as they took shape.

Furthermore, texture and materiality were added and constantly altered at every step. This 
ensured that the method was focused on the development of an architectural experience of space and not simply on the resolution of digital form.

The transformation of possible reality into real architectural possibility includes the incorporation of program and the restrictions of the physical site. The final form of the project is an apartment tower that also includes public functions and commercial space. The program is as follows:

\section{Residential:}

-60 apartment units

- lobby

- storage lockers

- laundry facilities

\section{Commercial:}

- café and convenience store

\section{Public/Cultural:}

- park space

- studio/gallery

The experiential conditions of the second model were taken as a qualitative program. The 
goal of this final stage was the integration of the quantitative program with these less tangible experiential elements. This experiential program focused on extracting various moments or conditions from the second model and resolving them architecturally in the project. The following qualitative program was developed from the experiences of the second model and their potential relationship to key elements of the quantitative program:

\section{Park:}

Spaces near the ground of the second model took on a very earthy, natural quality in the animations of that space. The extension of a natural element across the entire site is realized in the building in the form of a landscaped terrace that raises nature off of the ground plane of the site. Sculptural concrete masses separate the entrance of the building from the street, and are similar in texture and form to the elements in the experiential model from which they are inspired.

\section{Sky:}

The importance of the sky in the second model is evident in the many surfaces that either reflect or take on sky-like qualities. The animation of the sky gives life to these materials. In the apartments, windows are oriented primarily east an west, with solid walls oriented to catch the sky's early morning and late evening colors. The texture of the surface is animated naturally by the progression of the sun across the surface. 


\section{Solitude:}

A sense of quiet reflection permeates the experiential model. The building creates quiet, guarded corners for solitude in the gallery and on the accessible rooftop.

Further inspiration was drawn from other qualities of light and texture in the second model that inform the application of materiality in the final building. The animation of movement through the architectural model highlights several of these moments, which resist communication through static image. The final design is achieved by accessing the spaces of the model in duration. 


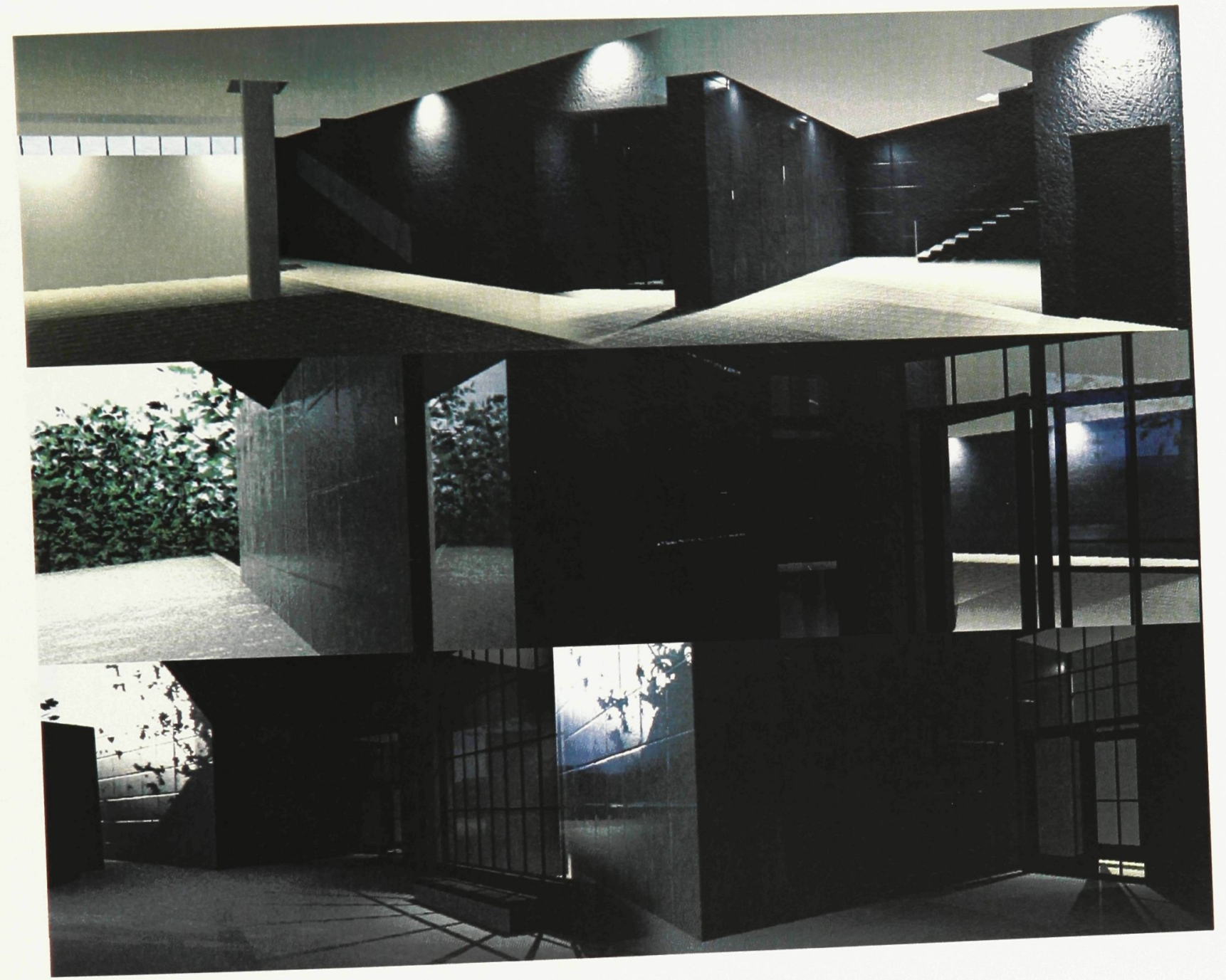

Fig. 42 Digital architectural model. 


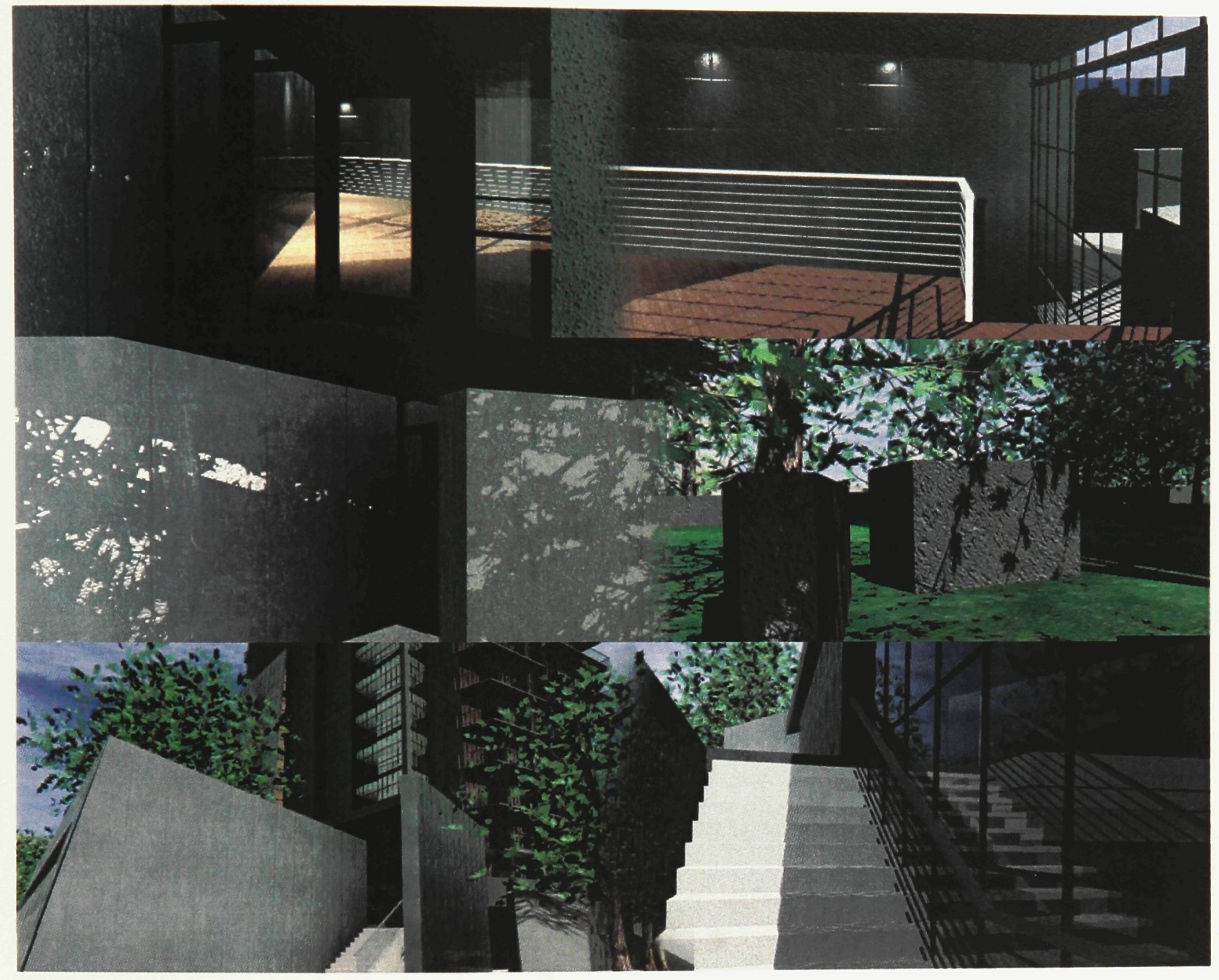

Fig. 43 Digital architectural model. 


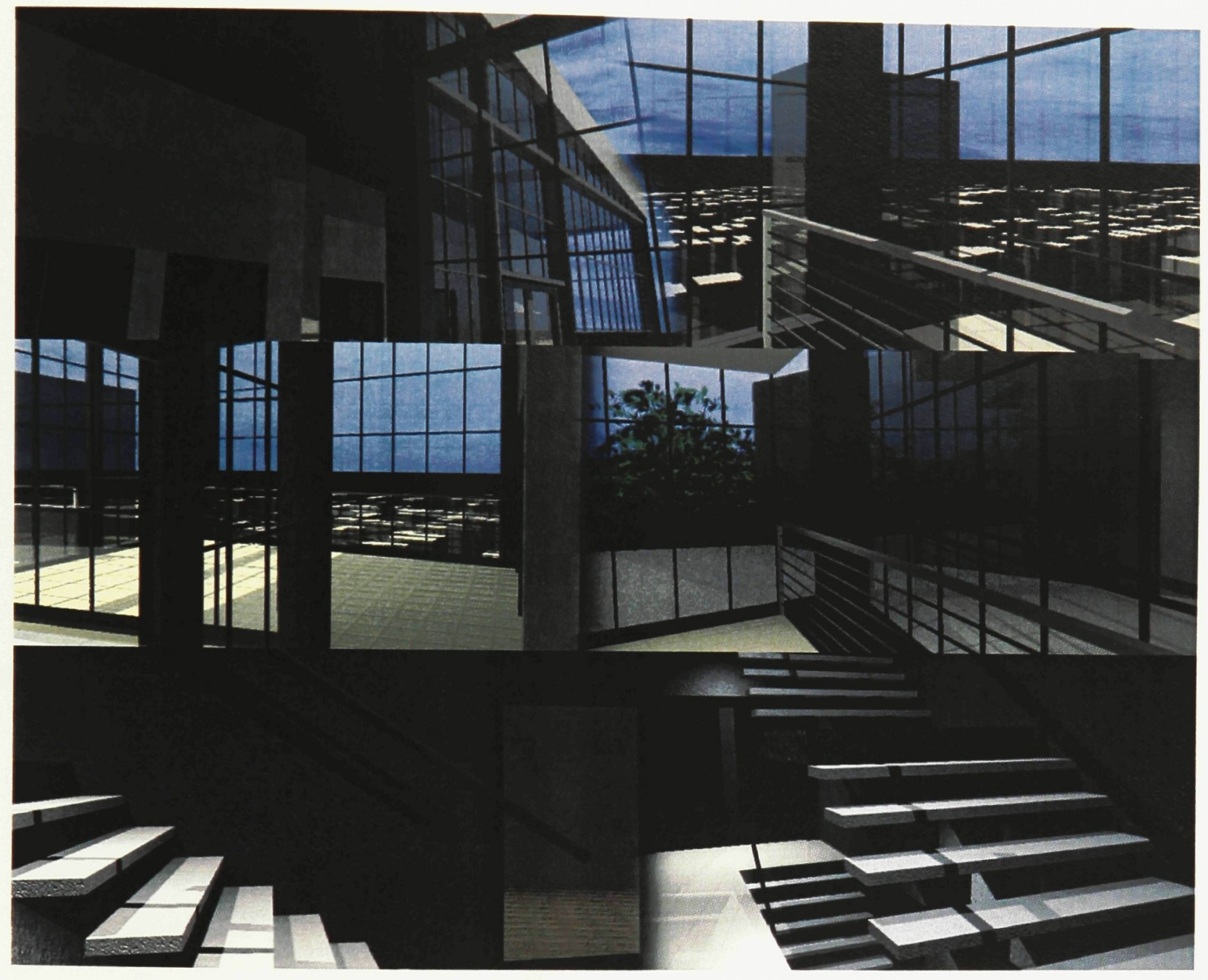

Fig. 44 Digital architectural model. 


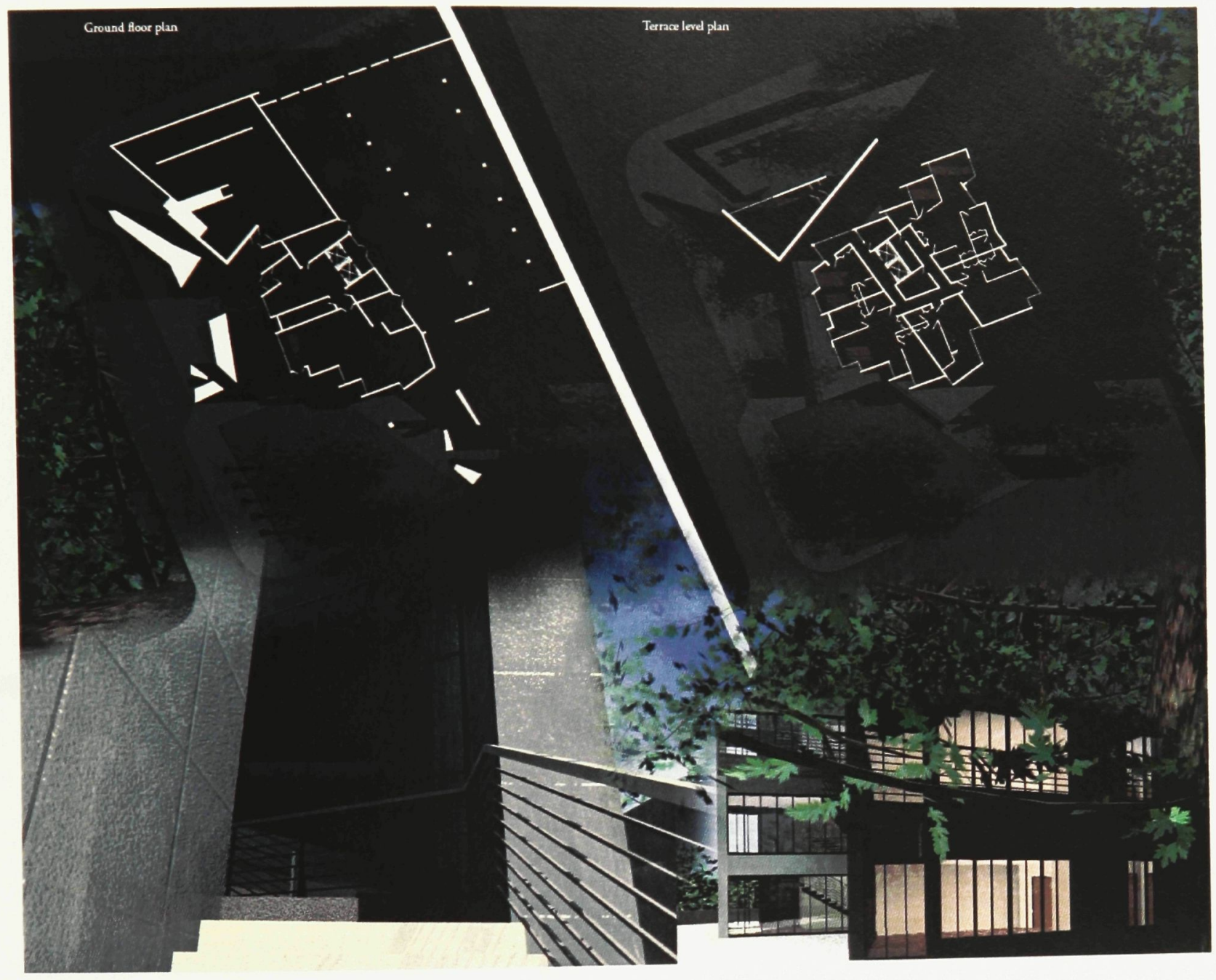

Fig. 45 Digital architectural model. 


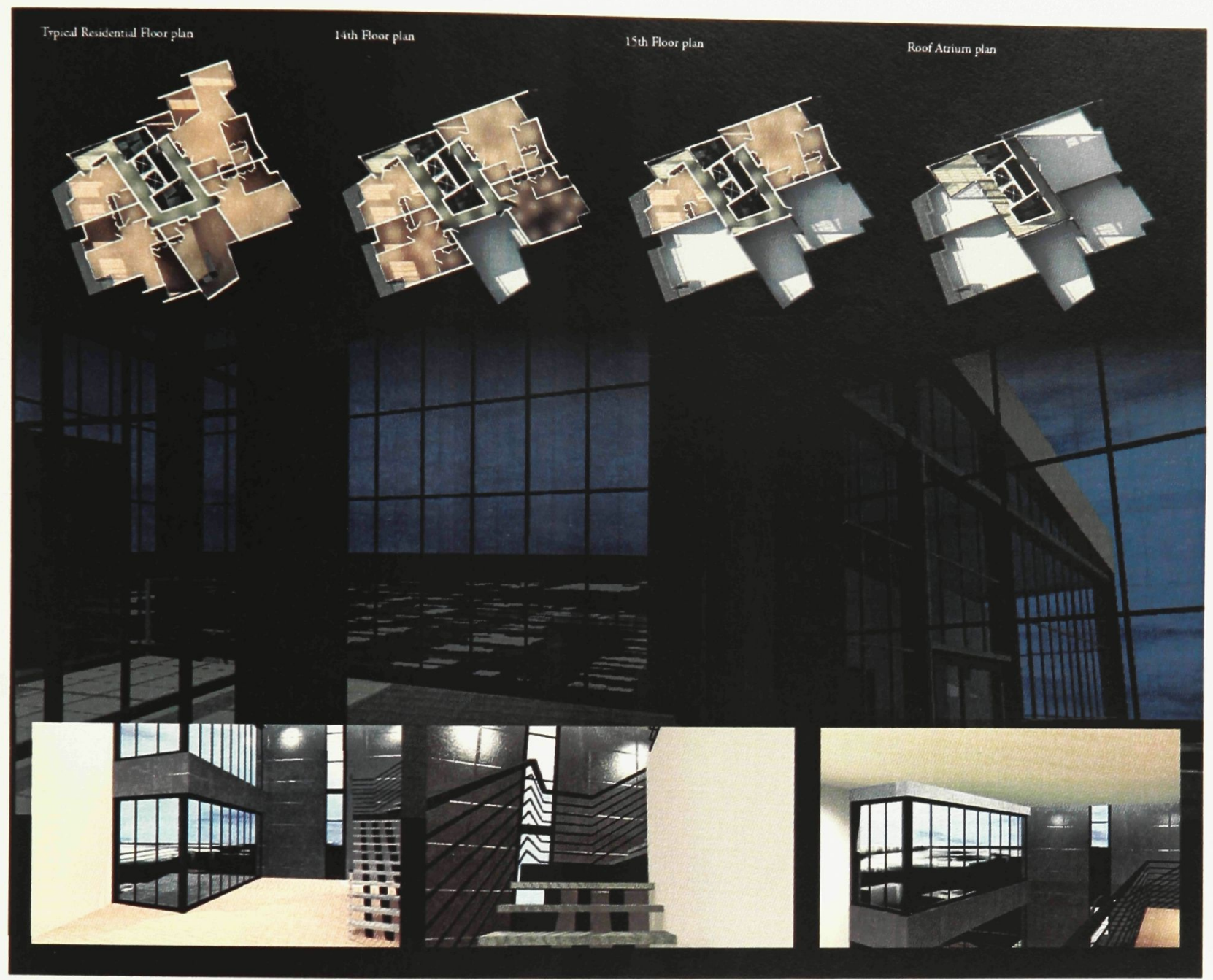

Fig. 46 Digital architectural model. 


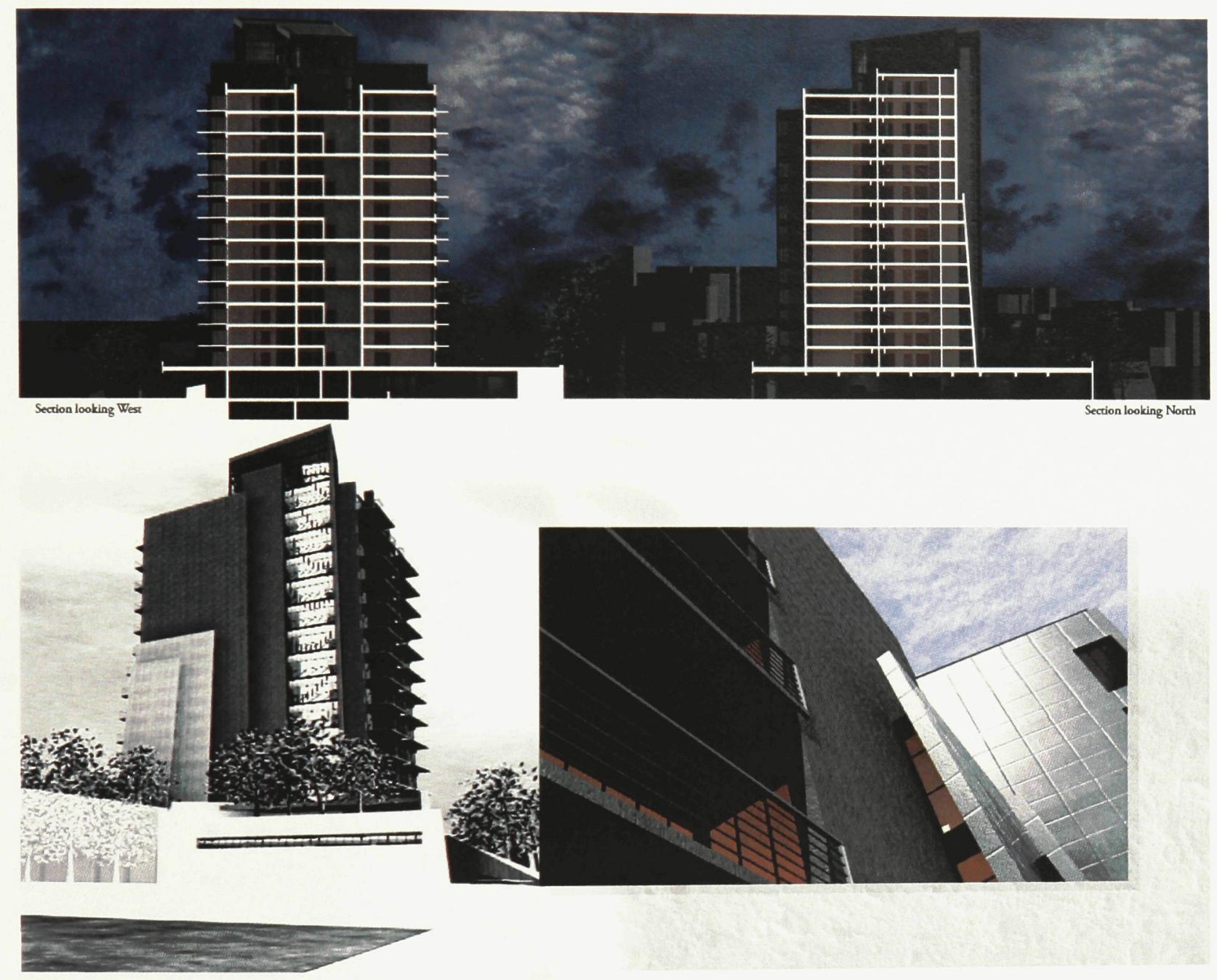

Fig. 47 Digital architectural model. 


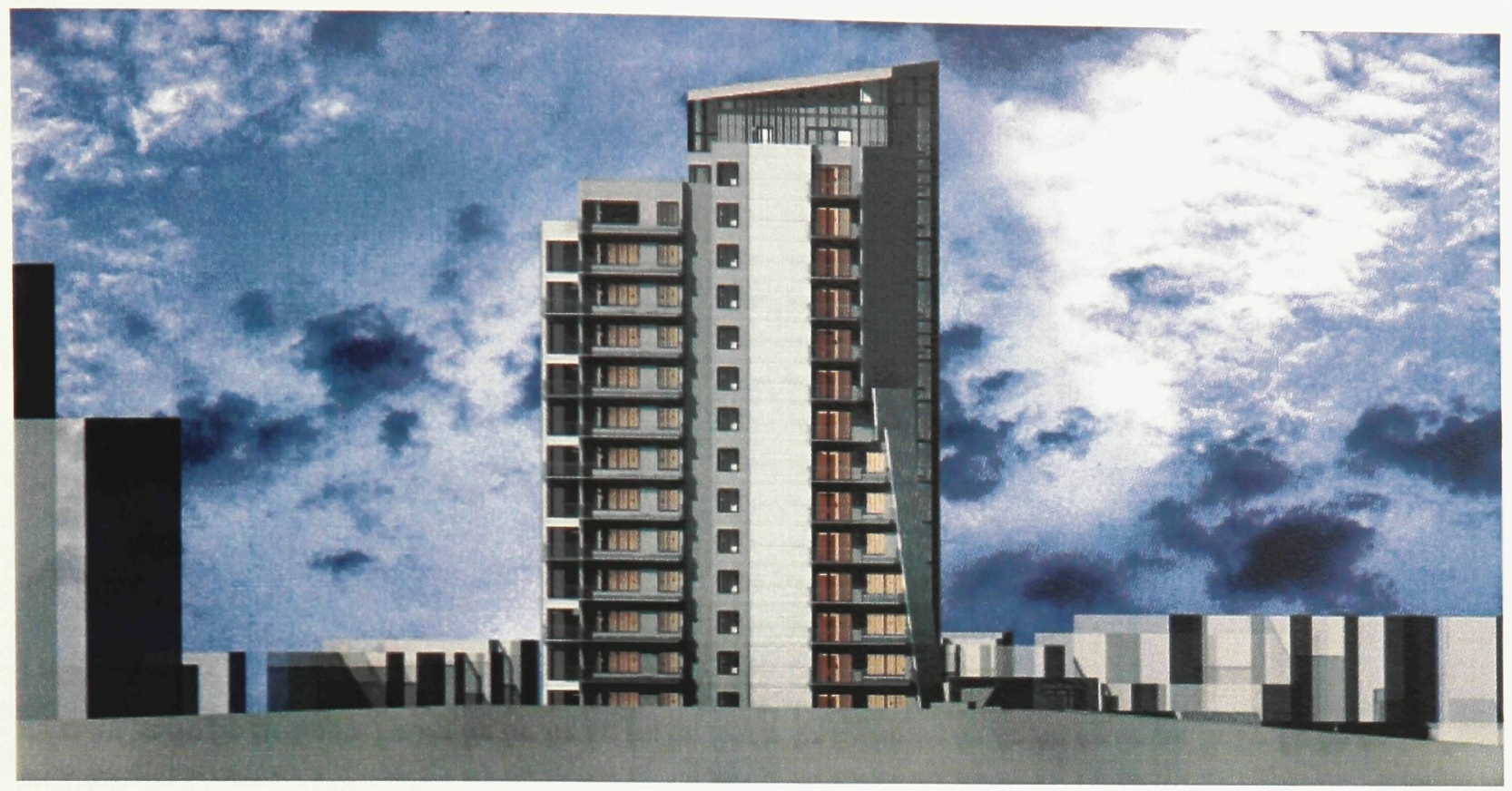

Fig. 48 East elevation. Digital architectural model.

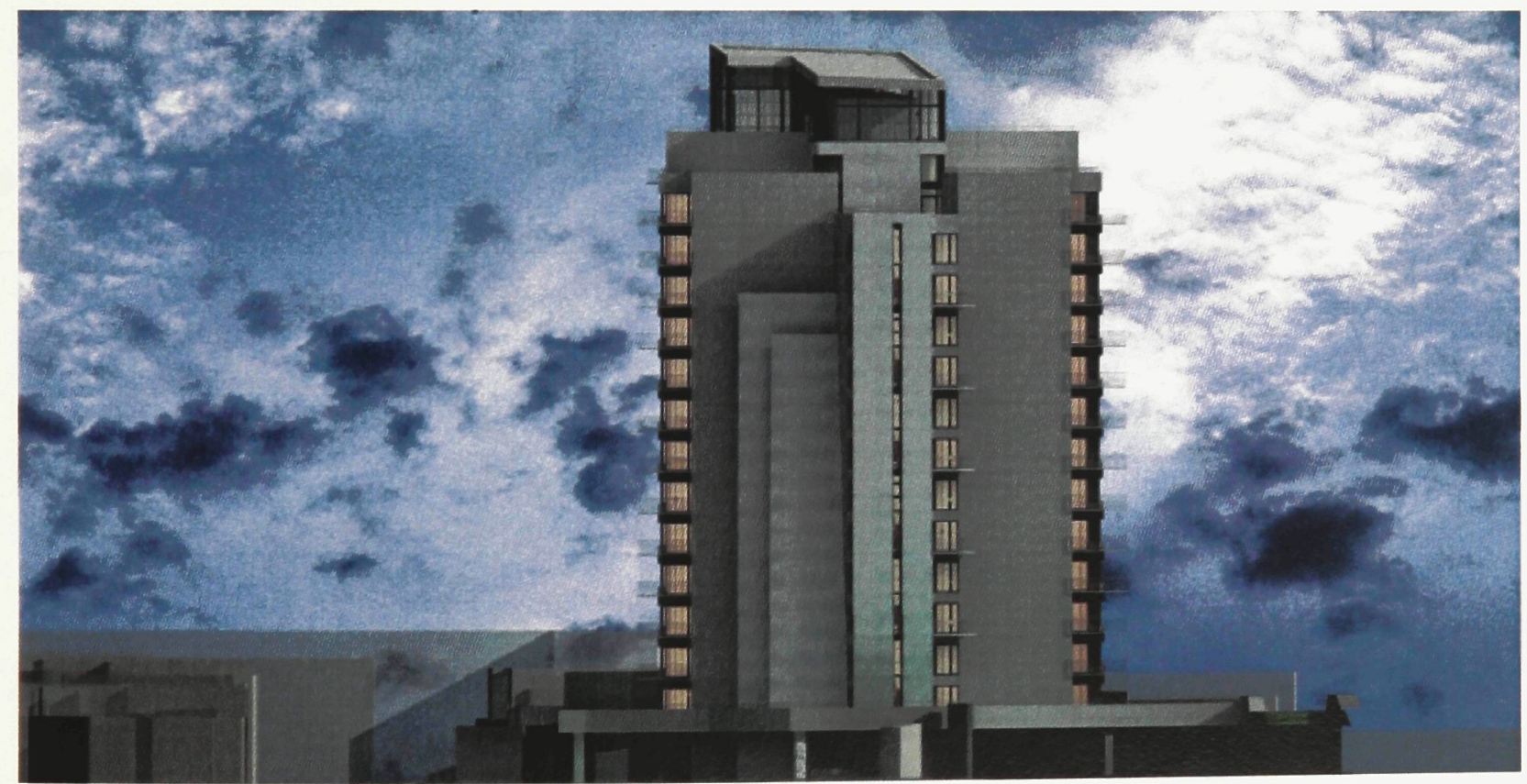

Fig. 49 South elevation. Digital architectural model. 


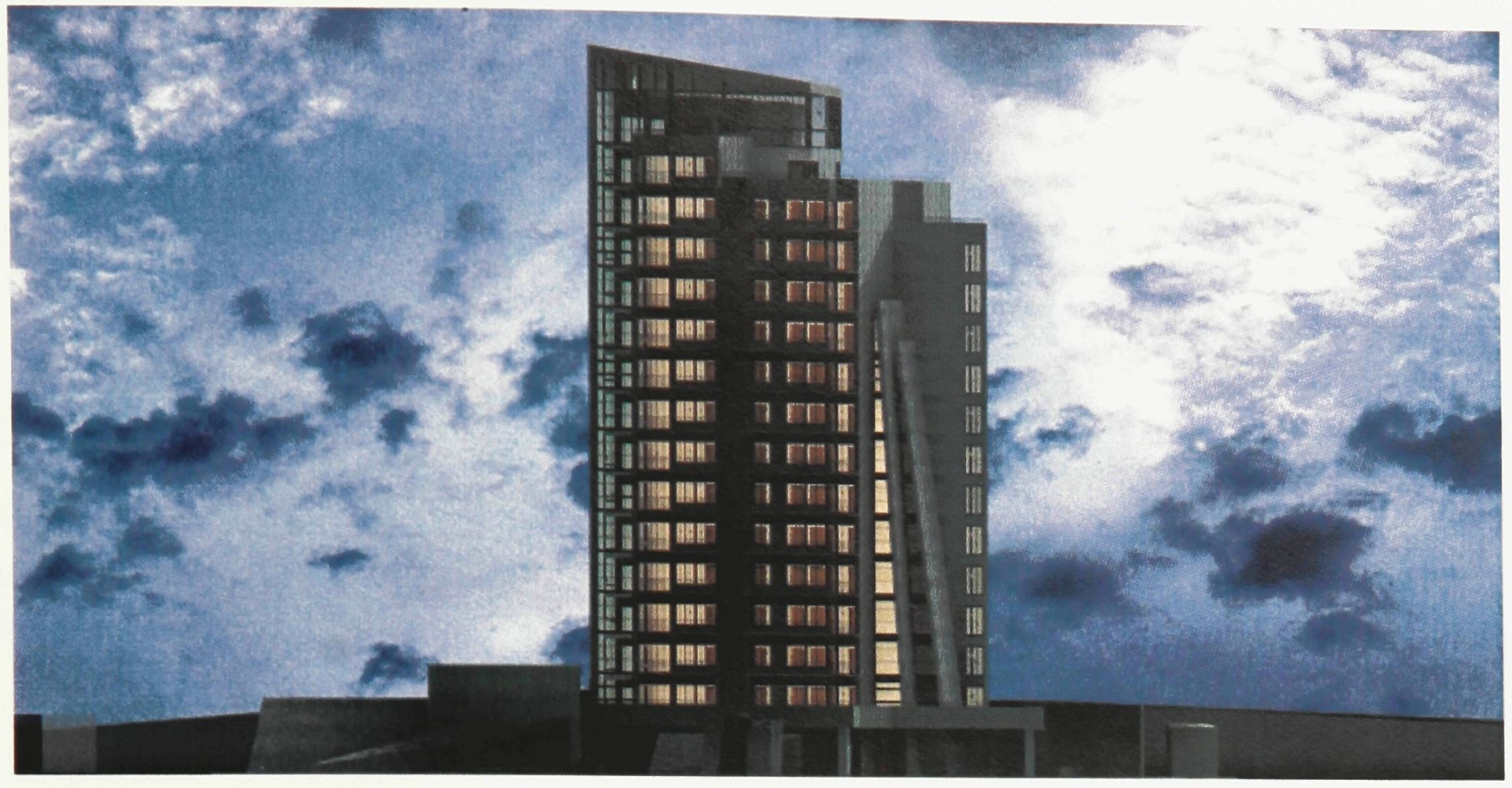

Fig. 50 West elevation. Digital architectural model.

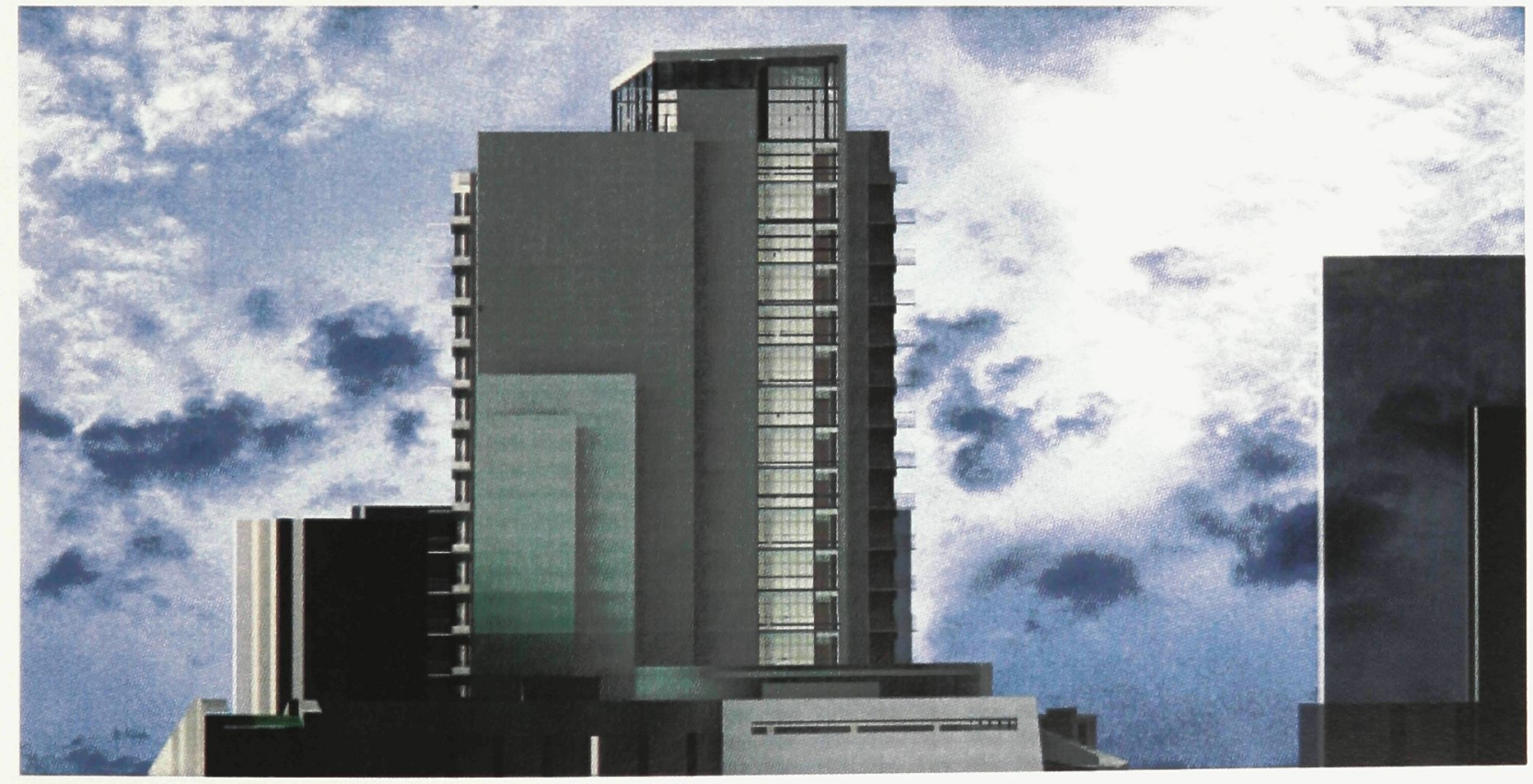

Fig. 51 North elevation. Digital architectural model. 


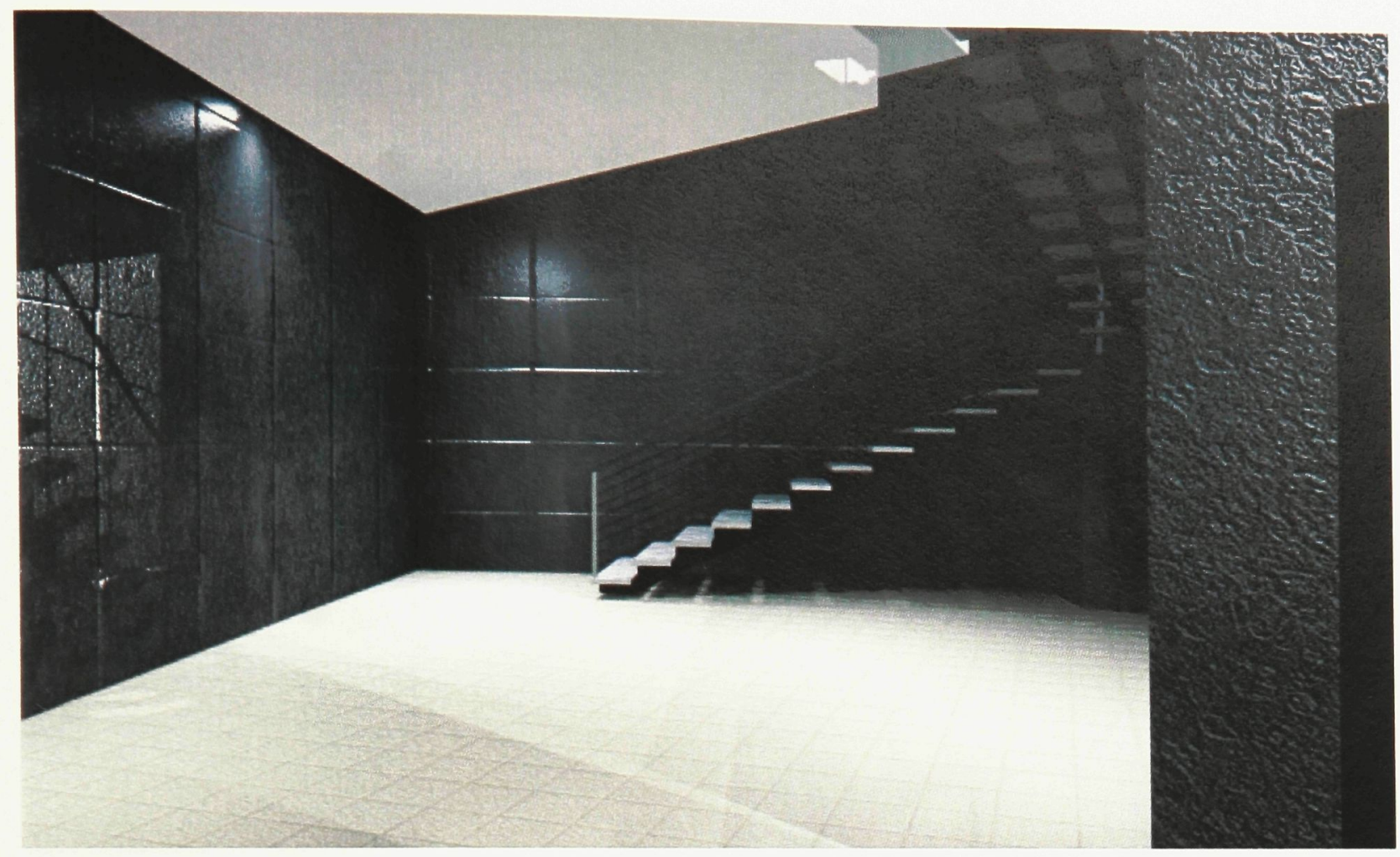

Fig. 52 Gallery interior. Digital architectural model.

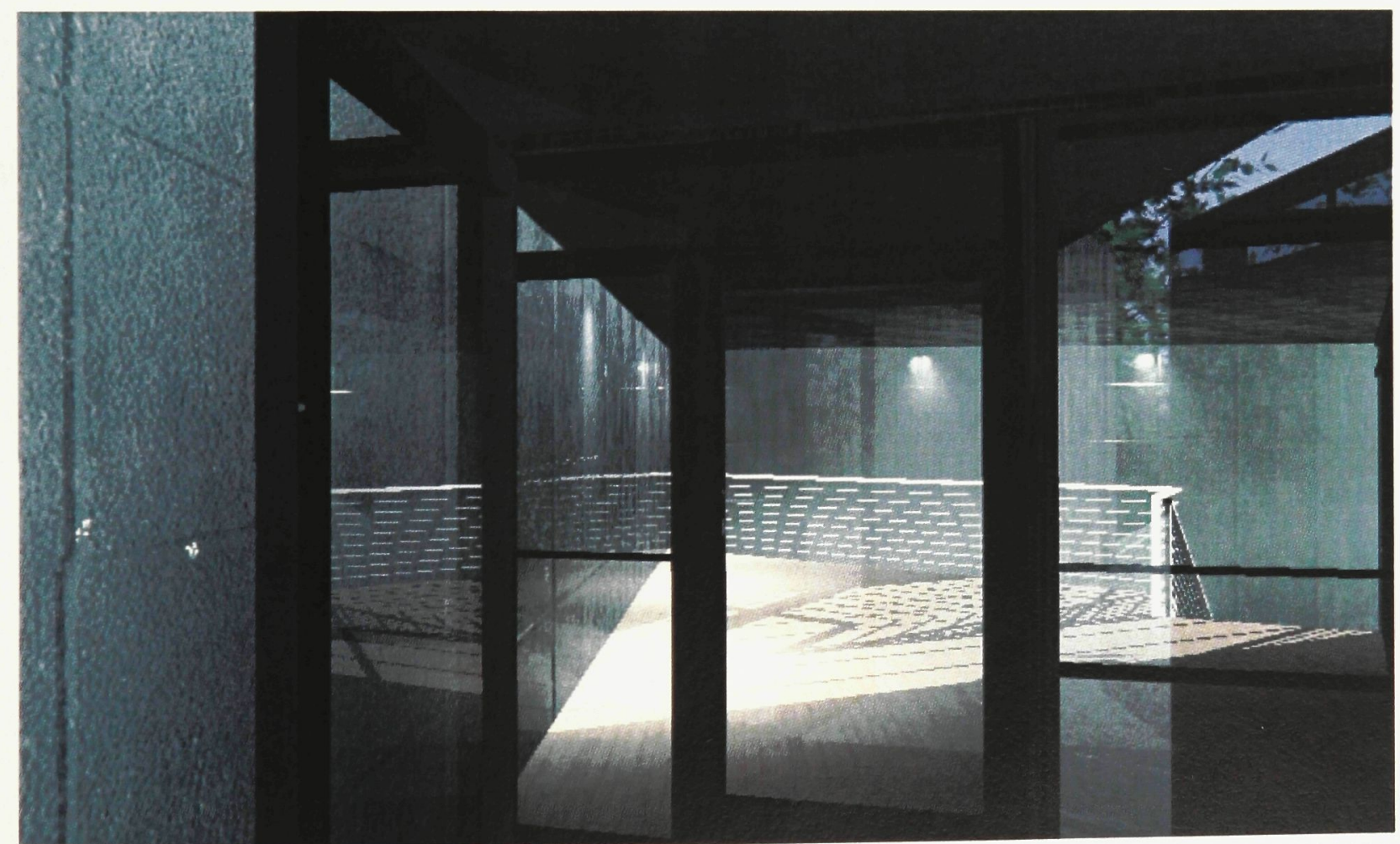

Fig. 53 Terrace level gallery entrance. Digital architectural model. 


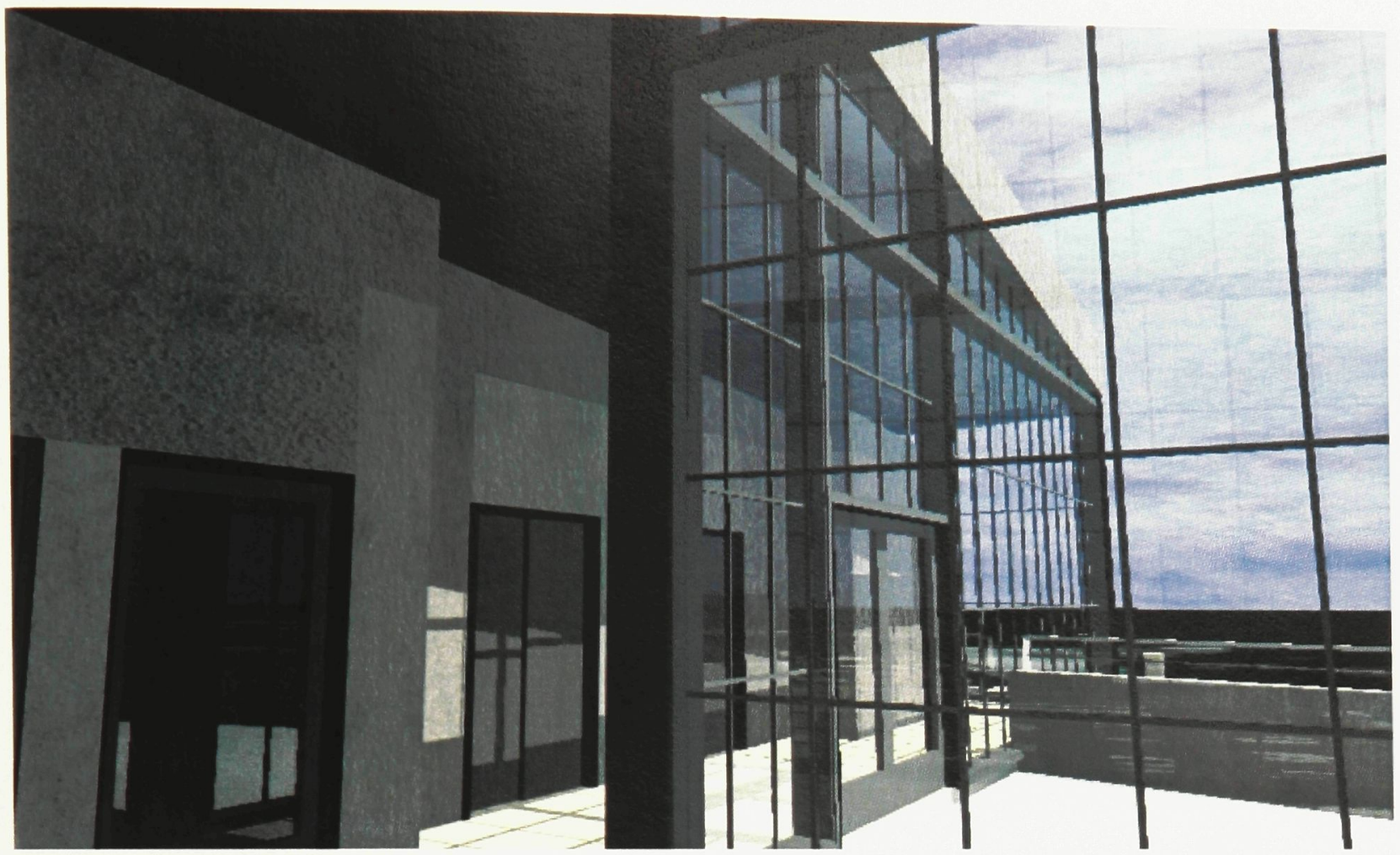

Fig. 54 Rooftop atrium. Digital architectural model.

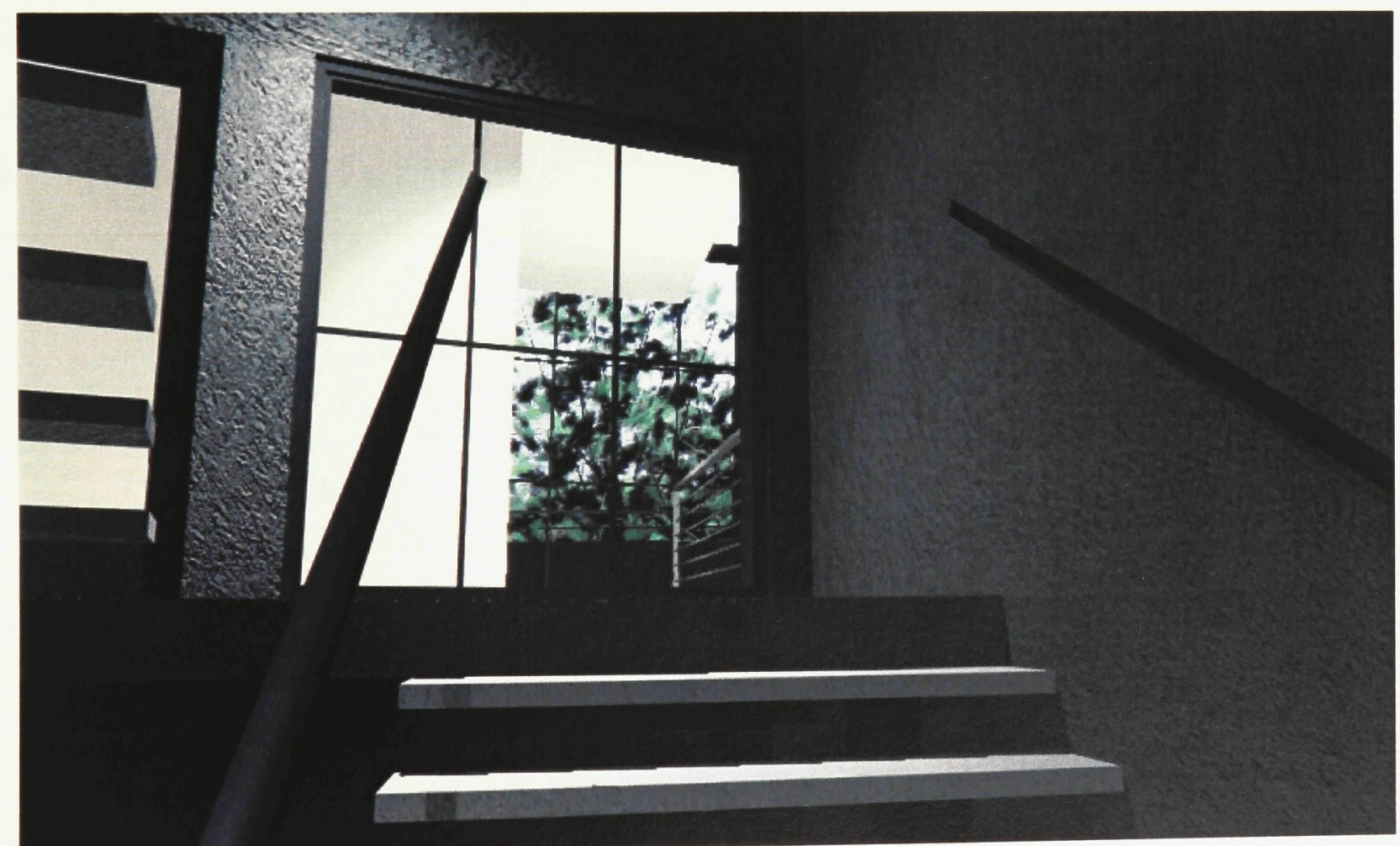

Fig. 55 Stairwell. Digital architectural model. 


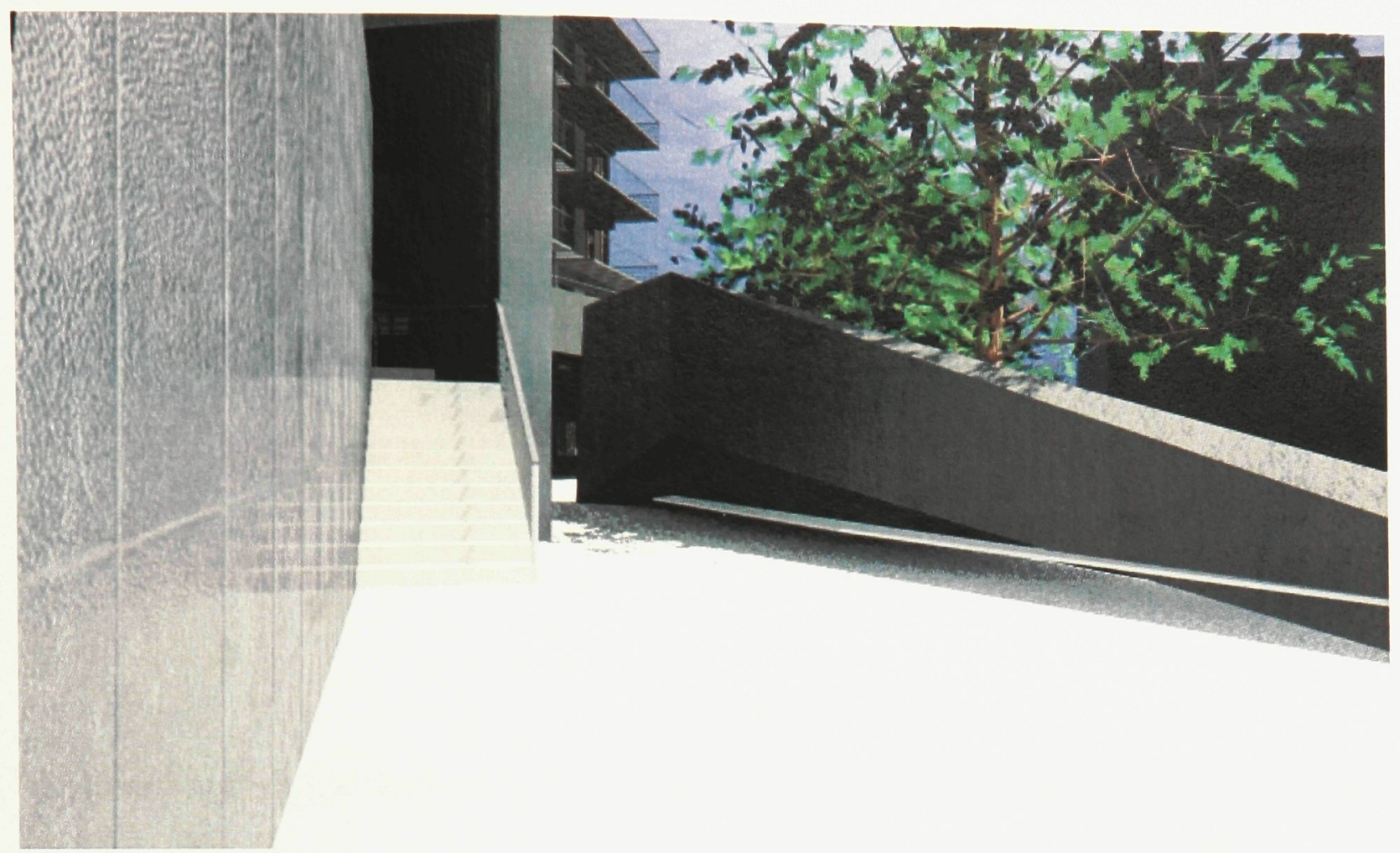

Fig. 56 Stair to terrace. Digital architectural model.

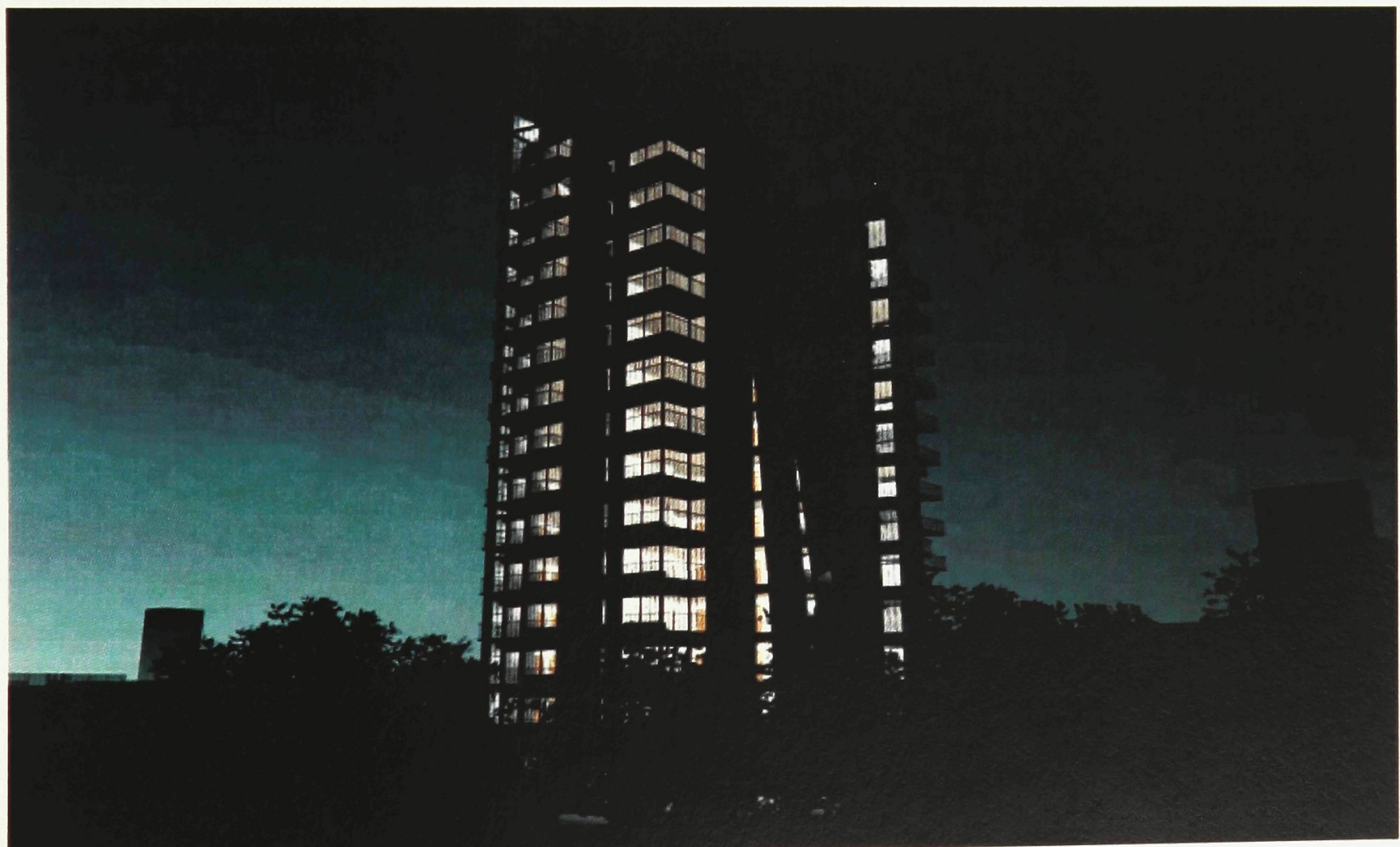

Fig. 57 Building at dusk. Digital architectural model. 


\section{Conclusion}

The insertion of digital media in the process of design as a tool for experiential exploration provides new ground for innovation in architecture; Grosz observes this as one of the fundamental effects of technological invention:

Although technology is in a sense made by us and for our purposes, it also performs a transformation on us: it increasingly facilitates not so much better action but wider possibilities of acting, more action. Technology is the great aid to action, for it facilitates, requires, and generates intelligence, which in turn radically multiplies our possibilities of action, our instrumental and practical relation with the world... ${ }^{50}$

Further to this, McLuhan's Laws of Media allow us to recognize those aspects of the design process that are augmented, enhanced, obsolesced, or retrieved through the digital medium; this enables us to explore the qualities of acceleration, simulation and animation that most significantly impact architectural representation.

\footnotetext{
50 Grosz, Architecture from the Outside, 177-178.
} 
The processes and results achieved in the theoretical projects of Lynn and Eisenman differ greatly in process and result from the exploration undertaken here, primarily because the digital tool in each case plays a slightly different role. In Lynn's work, for example, the space of the computer model is understood to be dynamic and reactionary, a field of forces and resultant deformation that takes its cues from application of digital modeling in other fields. Lynn explains:

Rather than as a frame through which time and space pass, architecture can be modeled as a participant immersed within dynamical flows. In addition to the special-effects and animation industries, many other disciplines such as aeronautical design, naval design, and automobile design employ this animate approach to modeling form in a space that is a medium of movement and force. ${ }^{51}$

Lynn uses the digital medium to investigate movement as it applies to the generation of form, and animation serves in his work to generate and reveal the transformations that occur. In Eisenman's "Virtual House" project, emphasis is placed on the recognition of progression that occurs in the deformation of the architecture. The computer is used to create this deformation, but the process is furthered through the assignment of program to the form that results. This final step in the "Virtual House" project is not dependant on the computer as a medium of representation, which distinctly separates it from the transformations that take place up to that point. Eisenman places little emphasis on the resolution of the virtual model into architectural convention. Because of this, the true project resides solely in the

\footnotetext{
${ }^{51}$ Lynn, Animate Form, 11.
} 
digital progression from cube and plane to the final shape of the house. Eisenman's focus on the digital medium in this project is inspired by Deleuze, who writes that "the virtual acquires full reality through being virtual." ${ }^{2}$

In this thesis exploration, the space of the computer model was not defined as a dynamic, force-driven environment, nor was it employed for the delineation of formal virtual transformations. Rather, the present focus emphasizes the qualities of animated virtual movement through cyberspace that facilitate projected inhabitation. The architectural experience is shaped through this inhabitation; because of this, the representations that result from the process of design are perspectival and interior in nature, unlike the exterior objectified vantage point given priority in Lynn and Eisenman's projects. The digital medium augmented my own ability to design in this manner, which proved to be a markedly different approach to my own past projects. What resulted was a project not radically different in form from my previous work, but much more refined in spatial and material quality. The privilege that I gave to the perspectival viewpoint allowed me to construct each space from within rather than from without. This mobile perspective emphasized resolution on a much smaller scale, and resulted in an architectural project that is richer in detail than in overall composition.

Since the primary goal of the thesis investigation is to insert the digital tool into the process of architectural design and discover its potential contributions, the selection of site and

\footnotetext{
$\overline{52}$ Deleuze as quoted in Galofaro, Digital Eisenman: An Office of the Electronic Era, 70.
} 
program seems at first to be an arbitrary condition. In selecting a mixed use residential program, the challenge was to apply the digital design process to a project type that, in practice, is constrained by considerations of cost, practicality and constructability. In retrospect, this program selection reveals an underlying, secondary agenda that is concerned with bridging the gap between theoretical architectural exploration and practical architectural construction. While this topic merits investigation in and of itself, it detracts in this instance from the main objective by introducing too many variables into the experiment. A large leap is made between the second and third models, which is a direct result of the inflexibility of a program based on typical floor plans and unit layouts. The rigid application of this program limited the expression of the experiential translation to the ground floor and roof of the building, and overshadowed a true investigation of the potential of the digital tool to affect architectural form. Future explorations should consider a program that is more flexible in its execution, which would allow it to be shaped by the application of the digital tool and the theories of virtual experience presented here, rather than acting as a restraining element on the same.

Program and site considerations aside, an evaluation of the use of the digital tool in the design process still highlights the three facets of the tool which are of interest. First, acceleration, which allows for the consideration of a great quantity of variation rapidly; indeed, the old adage that "quantity has a quality all its own" 53 lends credibility to this exercise. Secondly, simulation, which in the computer model describes a capacity for the

\footnotetext{
53 This phrase has been attributed to many sources, including Lenin, Stalin, and Mao.
} 
approximation of the real. Through experimentation with materiality and light as simulated entities, subtleties that evade recognition in traditional media are recognized and emphasized. Finally, animation has the greatest contribution to make to architectural expression because it facilitates the viewer's projected inhabitation in duration. Thus, it more effectively conveys a representation of experience to the viewer.

The integration of computer modeling into the architectural design process brings all of these enhanced qualities to bear on the final proposition. The translation of experiential representation into possible architectural reality is the final step in this integration.

Throughout the process, the virtual inhabitation of the space of the digital model allows for the development of an experience that is viewer oriented. In the end, the resulting architecture is enriched by the consideration throughout of this experiential potential, and the actualization of the virtual animated conditions in built form provides a point of reflection that further widens the scope of possible action. 


\section{Bibliography}

Alliez, Eric. “The Virtual Deleuze.” ANY, no. 19-20, 1997.

Barthes, Roland. "The Photographic Message". A Barthes Reader. Ed. Susan Sontag. New York: Hill and Wang, 1982.

Bergson, Henri. An Introduction to Metaphysics. United States: The Liberal Arts Press Inc., 1955.

---. Creative Evolution. Trans. Arthur Mitchell. London: MacMillan and Co. Limited, 1913.

---. Matter and Memory. Trans. N. M. Paul and W. S. Palmer. London: George Allen and Unwin Ltd., 1911.

---. Time and Free Will. Trans. F. L. Pogson. London: George Allen and Unwin Ltd., 1959.

Deleuze, Gilles. Bergsonism. Trans. H. Tomlinson and B. Habberjam. UrZone Inc., 1988

---. CINEMA 1, The Movement-Image. Trans. Hugh Tomlinson and Barbara Habberjam. Minneapolis: University of Minnesota Press, 1986.

Ednie-Brown, Pia. "The Texture of Diagrams". Daidalos. No. 74, 2000.

Eisenman, Peter. Diagram Diaries. New York: Universe Publishing, 1999.

---. "Visions unfolding: architecture in the age of electronic media". Domus, no. 734, 1992.

Evans, Robin." Translations from Drawing to Building". Translations from Drawing to Building and Other Essays. Cambridge, Massachusetts: The MIT Press, 1997. p. 
153-194.

Galofaro, Luca. Digital Eisenman: An Office of the Electronic Era. Trans. Lucinda Byatt.
Boston: Birkhäuser, 1999 .

Grosz, Elizabeth. Architecture from the Outside. Cambridge: The MIT Press, 2001. Hadid, Zaha. "Rosenthal Centre for Contemporary Art". August 16, $2005<$ http://www.
zaha-hadid.com/s.

Herzog, Jacques. "http://virtualhouse.ch.” ANY, no. 19-20, 1997.

Jasmann, Schawn. "Virtual Architecture and the Role of Inscription". Hybrid Reality: Art, Technology and the Human Factor. Ed. Hal Thwaites. Montreal, Canada: International Society on Virtual Systems and Multimedia, 2003.

Kant, Immanuel. Critique of Pure Reason. Trans. Wolfgang Schwarz. Germany: Scientin Verlag Aalen, 1982.

Kockelkoren, Petran. Technology: art, fairground and theatre. Rotterdam: NAi Publishers, 2003.

Lynn, Greg. Animate Form. New York: Princeton Architectural Press, 1999.

McLuhan, Marshall. Understanding Media: The Extensions of Man. New York: McGrawHill Book Company, 1964.

McLuhan, Eric and Marshall McLuhan. Laws of Media. Toronto: University of Toronto Press, 1988.

Nietzsche, Friedrich. "Twilight of the Idols". The Portable Nietzsche. Trans. Walter Kaufman. New York: Viking Penguin Inc., 1954. p.464-563.

Novak, Marcos. "Liquid Architecture in Cyberspace". Cyberspace: First Steps. Ed. Michael Benedikt, 228.

---. "Transarchitectures and Hypersurfaces - Operations of Transmodernity." Architectural Design. Vol. 68, no. 5-6, May-June 1998.

Perez-Gomez, Alberto. Architecture and the Crisis of Modern Science. Cambridge: The MIT Press, 1983.

Rajchman, John. "Artifice in an Ers@z World". ANY, no. 19-20, 1997.

Rocker, Ingeborg. "The Virtual: The Unform in Architecture". ANY, no. 19-20, 1997.

Trifonova, Temenuga. "Is There a Subject in Hyperreality?". POSTMODERN Culture. 2003. 9 Sept. 2005. <http://muse.jhu.edu/journals/postmodern_culture/v013/ 
13.3trifonova.html>

Virilio, Paul. "Cybernetics \& Society." ANY, no. 19-20, 1997. 